\title{
Reasoning with Very Expressive Fuzzy Description Logics
}

\author{
Giorgos Stoilos \\ Giorgos Stamou \\ Department of Electrical and Computer Engineering, \\ National and Technical University of Athens, \\ Zographou 15780, Athens, GR
}

Jeff Z. Pan

Department of Computing Science, The University of Aberdeen, UK

\section{Vassilis Tzouvaras}

Department of Electrical and Computer Engineering, National and Technical University of Athens, Zographou 15780, Athens, GR

\section{Ian Horrocks}

School of Computer Science, The University of Manchester

Manchester, M13 9PL, UK

\author{
GSTOIL@IMAGE.ECE.NTUA.GR
}

GSTAM@SOFTLAB.ECE.NTUA.GR

JPAN@CSD.ABDN.AC.UK

TZOUVARAS@IMAGE.ECE.NTUA.GR

HORROCKS@CS.MAN.AC.UK

\begin{abstract}
It is widely recognized today that the management of imprecision and vagueness will yield more intelligent and realistic knowledge-based applications. Description Logics (DLs) are a family of knowledge representation languages that have gained considerable attention the last decade, mainly due to their decidability and the existence of empirically high performance of reasoning algorithms. In this paper, we extend the well known fuzzy $\mathcal{A} \mathcal{L C}$ DL to the fuzzy $\mathcal{S H I N}$ DL, which extends the fuzzy $\mathcal{A L C}$ DL with transitive role axioms $(\mathcal{S})$, inverse roles $(\mathcal{I})$, role hierarchies $(\mathcal{H})$ and number restrictions $(\mathcal{N})$. We illustrate why transitive role axioms are difficult to handle in the presence of fuzzy interpretations and how to handle them properly. Then we extend these results by adding role hierarchies and finally number restrictions. The main contributions of the paper are the decidability proof

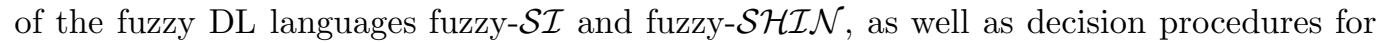
the knowledge base satisfiability problem of the fuzzy-SI and fuzzy-SHIN .
\end{abstract}

\section{Introduction}

Nowadays, many applications and domains use some form of knowledge representation language in order to improve their capabilities. Encoding human knowledge and providing means to reason with it can benefit applications a lot, by enabling them provide intelligent answers to complex user defined tasks. Examples of modern applications that have recently adopted knowledge representation languages are the World Wide Web (BernersLee, Hendler, \& Lassila, 2001; Baader, Horrocks, \& Sattler, 2002b), where knowledge is used to improve the abilities of agents and the interoperability between disparate systems, multimedia processing applications (Alejandro, Belle, \& Smith, 2003; Benitez, Smith, \& Chang, 2000), which use knowledge in order to bridge the "gap" between human percep- 
tion of the objects that exist within multimedia documents, and computer "perception" of pixel values, configuration applications (McGuiness, 2003), etc. Unfortunately, there are occasions where traditional knowledge representation languages fail to accurately represent the concepts that appear in a domain of interest. For example, this is particularly the case when domain knowledge is inherently imprecise or vague. Concepts like that of a "near" destination (Berners-Lee et al., 2001), a "highQuality" audio system (McGuiness, 2003), "many" children, a "faulty" reactor (Horrocks \& Sattler, 1999), "soon" and many more, require special modelling features. In the past many applications of various research areas, like decision making, image processing, robotics and medical diagnosis have adopted special mathematical frameworks that are intended for modelling such types of concepts (Zimmermann, 1987; Larsen \& Yager, 1993; Krishnapuram \& Keller, 1992). One such a mathematical framework is fuzzy set theory (Zadeh, 1965). Though fuzzy extensions of various logical formalisms, like propositional, predicate or modal logics have been investigated in the past (Hajek, 1998), such a framework is is not yet well developed for Description Logics and much research work needs to be done. More precisely, there is the need for reasoning in very expressive fuzzy Description Logics.

In order to achieve knowledge reusability and high interoperability, modern applications often use the concept of an "ontology" (Berners-Lee et al., 2001) to represent the knowledge that exists within their domain. Ontologies are created by encoding the full knowledge we possess for a specific entity of our world using a knowledge representation language. A logical formalism that has gained considerable attention the last decade is Description Logics (Baader, McGuinness, Nardi, \& Patel-Schneider, 2002a). Description Logics (DLs) are a family of class-based (concept-based) knowledge representation formalisms, equipped with well defined model-theoretic semantics (Tarski, 1956). They are characterized by the use of various constructors to build complex concept descriptions from simpler ones, an emphasis on the decidability of key reasoning problems, and by the provision of sound, complete and empirically tractable reasoning services. Both the well-defined semantics and the powerful reasoning tools that exist for Description Logics makes them ideal for encoding knowledge in many applications like the Semantic Web (Baader et al., 2002b; Pan, 2004), multimedia applications (Meghini, Sebastiani, \& Straccia, 2001), medical applications (Rector \& Horrocks, 1997), databases (Calvanese, De Giacomo, Lenzerini, Nardi, \& Rosati, 1998) and many more. Interestingly, the current standard for Semantic Web ontology languages, OWL (Bechhofer, van Harmelen, Hendler, Horrocks, McGuinness, Patel-Schneider, \& eds., 2004), is based on Description Logics to represent knowledge and support a wide range of reasoning services. More precisely, without regarding annotation properties of OWL, the OWL Lite species of OWL is equivalent to the $\mathcal{S H \mathcal { H }} \mathcal{F}\left(\mathbf{D}^{+}\right)$DL, while OWL DL is equivalent to $\mathcal{S H O I N}\left(\mathbf{D}^{+}\right)$(Horrocks, Patel-Schneider, \& van Harmelen, 2003). Although DLs provide considerable expressive power, they feature limitations regarding their ability to represent vague (fuzzy) knowledge. As obvious, in order to make applications that use DLs able to cope with such information we have to extend them with a theory capable of representing such kind of information. One such important theory is fuzzy set theory. Fuzzy Description Logics are very interesting logical formalisms as they can be used in numerous domains like multimedia and information retrieval (Fagin, 1998; Meghini et al., 2001) to provide ranking degrees, geospatial (Chen, Fellah, \& Bishr, 2005) to cope with vague concepts like "near", "far" and many more. 
In order to make the need to handle vagueness knowledge more evident and the application of fuzzy set theory more intuitive, let us consider an example. Suppose that we are creating a knowledge-based image processing application. In such application the task is to (semi)automatically detect and recognize image objects. Suppose also that the content of the images represents humans or animals. For such a domain one can use standard features of Description Logics to encode knowledge. For example, a knowledge base describing human bodies could contain the following entities

$$
\begin{gathered}
\text { Arm } \sqsubseteq \exists \text { isPartOf.Body } \\
\text { Body } \sqsubseteq \exists \text { isPartOf.Human }
\end{gathered}
$$

where $\sqsubseteq$ is a subsumption relation and isPartOf is obviously a transitive relation. This knowledge can be captured with the aid of the $\mathcal{S}$ DL (Sattler, 1996). Moreover, one might want to capture the knowledge that the role hasPart is the inverse of the role isPartOf, writing hasPart $:=$ isPartOf $^{-}$, thus being able to state that something that is a body and has a tail is also an animal as,

\section{Body $\sqcap$ ヨhasPart. Tail $\sqsubseteq$ Animal.}

For this new feature one would require the $\mathcal{S I}$ DL (Horrocks \& Sattler, 1999). The new axiom gives us the ability to recognize that the concept Arm $\sqcap$ Tail is subsumed by $\exists$ isPartOf.Animal. Finally, the $\mathcal{S I}$ DL can be further extended with role hierarchies and number restrictions. Hence, one is able to capture the fact that the role hasDirectPart is a sub-role of the role hasPart, by writing isDirectPartOf $\sqsubseteq$ isPartOf, while we can also provide a more accurate definition of the concept Body by giving the axiom,

$$
\text { Body } \sqsubseteq \exists \text { isDirectPartOf.Human } \sqcap \leq 2 \text { hasArm } \sqcap \geq 2 \text { hasArm }
$$

stating that the body is a direct part of a human and it also has exactly two arms.

Up to now we have only used standard Description Logic features. Now suppose that we run an image analysis algorithm. Such algorithms usually segment the image into regions and try to annotate them with appropriate semantic labels using low level image features. This process involves a number of vague concepts since an image region might be red, blue, circular, small or smooth textured to some degree or two image regions might not be totally but only to some degree adjacent (since not all of their pixels are adjacent), one contained within the other, etc. Hence we can only decide about the membership of a region to a specific concept only to a certain degree (Athanasiadis, Mylonas, Avrithis, $\&$ Kollias, 2007). For example, in our case we could have that the object $o_{1}$ isPartOf the object $o_{2}$ to a degree of 0.8 , that $o_{2}$ isPartOf $o_{3}$ to a degree of 0.9 , that $o_{1}$ is an Arm to a degree of 0.75 and that $\mathrm{O}_{2}$ is a Body to a degree of 0.85. From that fuzzy knowledge one could deduce that $o_{3}$ belongs to the concept $\exists$ hasPart.Body $\sqcap \exists$ hasPart.Arm to a degree of 0.75. This together with a definition of the form Human $\equiv \exists$ hasPart.Body $\sqcap \exists$ hasPart.Arm, where $\equiv$ represents equivalence, means that there is a good chance that $o_{3}$ is a Human. Observe, that in this definition, in order for someone to be a human, we do not force a Body to explicitly have a part that is an Arm. This is a reasonable choice in the present application, because depending on the level of the segmentation, there might be several 
segmented regions between $o_{2}$ and $o_{3}$. As it is obvious is such applications handling the inherent vagueness certainly benefits the specific application.

In this paper we extend the well known fuzzy $\mathcal{A L C}(\mathrm{f}-\mathcal{A L C}) \mathrm{DL}($ Straccia, 2001) to the fuzzy $\mathcal{S H} \mathcal{H} \mathcal{N}$ DL $(\mathrm{f}-\mathcal{S H} \mathcal{H} \mathcal{N})$, which extends the f- $\mathcal{A L C}$ DL with the inverse role constructor, transitive role axioms, role hierarchies and the number restrictions constructor. Moreover, we prove the decidability of the $\mathrm{f}-\mathcal{S H \mathcal { N } N}$ DL by providing a tableaux algorithm for deciding the standard DL inference problems. In order to provide such an algorithm we proceed in two steps. First, we focus on the $\mathrm{f}-\mathcal{S I}$ language studying the properties of fuzzy transitive roles in value and existential restrictions, as well as the applicability of the techniques used in the classical $\mathcal{S I}$ language to ensure the termination of the algorithm (Horrocks \& Sattler, 1999). As we will see there is great difficulty on handling such axioms on the context of fuzzy DLs, but after finishing our investigation we will see that similar notions as in classical $\mathcal{S} \mathcal{I}$ language can be applied. Secondly, we extend these results by adding role hierarchies and number restrictions. We provide all the necessary extensions to the reasoning algorithm of

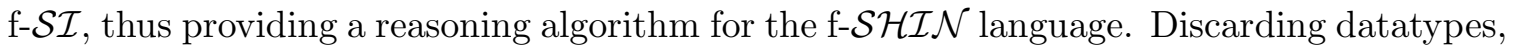
$\mathcal{S H \mathcal { I N }}$ is slightly more expressive than $\mathcal{S H \mathcal { H } F}$ (OWL Lite) and slightly less expressive than $\mathcal{S H O I N}(O W L \mathrm{DL})$. In order to achieve our goal we again extend the techniques used for the classical $\mathcal{S H \mathcal { I N }}$ language and which ensure correctness of the algorithm (Horrocks \& Sattler, 1999; Horrocks, Sattler, \& Tobies, 2000). Finally, we prove the decidability of the extended algorithm. There are many benefits on following such an approach. On the one hand we provide a gradual presentation to the very complex algorithm of $\mathrm{f}-\mathcal{S H \mathcal { I N }}$, while on the other hand we provide a reasoning algorithm for a less expressive, but more efficient fuzzy DL language, f-SII. The classical $\mathcal{S I}$ language is known to be PSPACE-complete, in contrast to the Exptime-completeness of $\mathcal{S H \mathcal { I N }}$ (Tobies, 2001), hence our algorithm for f-SI can be used for future research and for providing efficient and optimized implementations.

Please note that fuzzy DLs (Straccia, 2001) are complementary to other approaches that extend DLs, like probabilistic DLs (Koller, Levy, \& Pfeffer, 1997; Giugno \& Lukasiewicz, 2002; Ding \& Peng, 2004), or possibilistic DLs (Hollunder, 1994). More precisely, these theories are meant to be used for capturing different types of imperfect information and knowledge. Fuzziness is purposed for capturing vague (fuzzy) knowledge, i.e. facts that are certain but which have degrees of truth assigned to them, like for example the degree of truth of someone being tall. On the other hand, possibilistic and probabilistic logics are purposed for capturing cases where knowledge is uncertain due to lack of information or knowledge about a specific situation or a future event, like for example a sensor reading or a weather forecast. These facts are assigned degrees of possibility, belief or probability, rather than truth degrees. Dubois and Prade (2001) provide a comprehensive analysis on these theories along with their different properties.

The rest of the paper is organized as follows. Section 2 briefly introduces the DL $\mathcal{S H \mathcal { N }}$ and provides some preliminaries about the notion of a fuzzy set and how set theoretic and logical operations have been extended to the fuzzy set framework. Section 3 introduces the

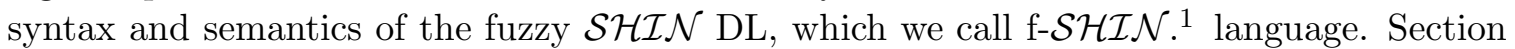
4 provides an investigation on the semantics of fuzzy DLs when fuzzy transitive relations

1. In a previous approach to fuzzy DLs the notation $f \mathcal{A L C}$ is used (Straccia, 2004), but this notation is not so flexible to represent fuzzy DLs which use different norm operations, as we will see later on. In some other approaches (Tresp \& Molitor, 1998; Hölldobler, Khang, \& Störr, 2002) the naming $\mathcal{A} \mathcal{L C}_{F}$ is 
participate in value and existential restrictions. In section 5 we give a detailed presentation of the reasoning algorithm for deciding the consistency of a fuzzy-SI ABox and we provide the proofs for the termination, soundness and completeness of the procedure. Then, in section 6 we extend the previous results by adding role hierarchies and number restrictions. More precisely, the results of section 4 are enriched by considering transitive roles and roles hierarchies in value and existential restrictions. Using this results we extend the algorithm of section 5 to handle with these new feature and finally we prove its soundness, completeness and termination. At last, in section 7 we review some previous work on fuzzy Description Logics while section 8 concludes the paper.

\section{Preliminaries}

In the current section we will briefly introduce classical DLs and fuzzy set theory, recalling some mathematical properties of fuzzy set theoretic operators.

\subsection{Description Logics and the $\mathcal{S H \mathcal { I N }}$ DL}

Description Logics (DLs) (Baader et al., 2002a) are a family of logic-based knowledge representation formalisms designed to represent and reason about the knowledge of an application domain in a structured and well-understood way. They are based on a common family of languages, called description languages, which provide a set of constructors to build concept (class) and role (property) descriptions. Such descriptions can be used in axioms and assertions of DL knowledge bases and can be reasoned about with respect to (w.r.t.) DL knowledge bases by DL systems.

In this section, we will briefly introduce the $\mathcal{S H \mathcal { I N }} \mathrm{DL}$, which will be extended to the f-SHIN DL later. A description language consists of an alphabet of distinct concept names $(\mathbf{C})$, role names $(\mathbf{R})$ and individual (object) names $(\mathbf{I})$; together with a set of constructors to construct concept and role descriptions.

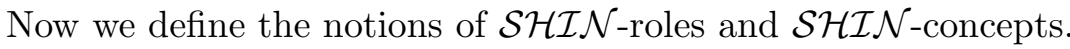

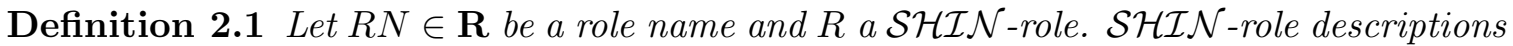

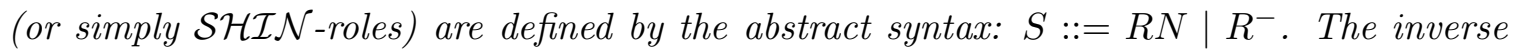
relation of roles is symmetric, and to avoid considering roles such as $R^{--}$, we define a function Inv which returns the inverse of a role, more precisely,

$$
\operatorname{lnv}(R):= \begin{cases}R N^{-} & \text {if } R=R N \\ R N & \text { if } R=R N^{-}\end{cases}
$$

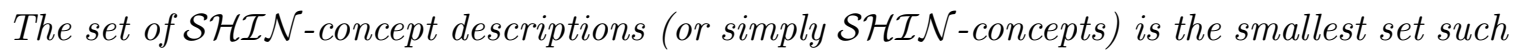
that:

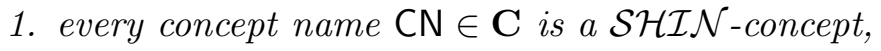

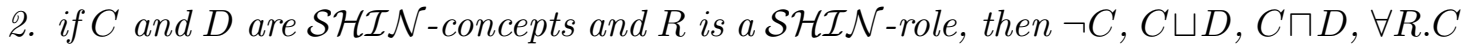
and $\exists R . C$ are also $\mathcal{S H I N}$-concepts, called general negation (or simply negation),

used but this can easily be confused with $\mathcal{A L C F}(\mathcal{A L C}$ extended with functional restrictions, Horrocks \& Sattler, 1999), when pronounced. 


\begin{tabular}{|l|c|l|}
\hline \multicolumn{1}{|c|}{ Constructor } & Syntax & \multicolumn{1}{|c|}{ Semantics } \\
\hline top & $\top$ & $\Delta^{\mathcal{I}}$ \\
\hline bottom & $\perp$ & $\emptyset$ \\
\hline concept name & $\mathrm{CN}$ & $\mathrm{CN}^{\mathcal{I}} \subseteq \Delta^{\mathcal{I}}$ \\
\hline general negation & $\neg C$ & $\Delta^{\mathcal{I}} \backslash C^{\mathcal{I}}$ \\
\hline conjunction & $C \sqcap D$ & $C^{\mathcal{I}} \cap D^{\mathcal{I}}$ \\
\hline disjunction & $C \sqcup D$ & $C^{\mathcal{I}} \cup D^{\mathcal{I}}$ \\
\hline exists restriction & $\exists R . C$ & $\left\{x \in \Delta^{\mathcal{I}} \mid \exists y .\langle x, y\rangle \in R^{\mathcal{I}} \wedge y \in C^{\mathcal{I}}\right\}$ \\
\hline value restriction & $\forall R . C$ & $\left\{x \in \Delta^{\mathcal{I}} \mid \forall y .\langle x, y\rangle \in R^{\mathcal{I}} \rightarrow y \in C^{\mathcal{I}}\right\}$ \\
\hline at-most restriction & $\leq n R$ & $\left\{x \in \Delta^{\mathcal{I}} \mid \sharp\left\{y \in \Delta^{\mathcal{I}} \mid R^{\mathcal{I}}(x, y)\right\} \leq n\right\}$ \\
\hline at-least restriction & $\geq n R$ & $\left\{x \in \Delta^{\mathcal{I}} \mid \sharp\left\{y \in \Delta^{\mathcal{I}} \mid R^{\mathcal{I}}(x, y)\right\} \geq n\right\}$ \\
\hline
\end{tabular}

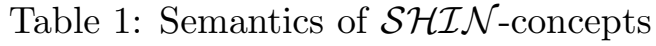

disjunction, conjunction, value restrictions and existential restriction, respectively, and

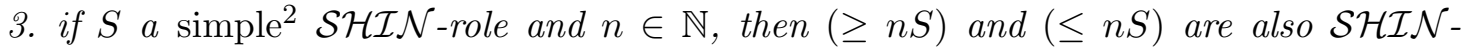
concepts, called at-most and at-least number restrictions.

By removing point 3 of the above definition we obtain the set of $\mathcal{S I}$-concepts.

Description Logics have a model-theoretic semantics, which is defined in terms of interpretations. An interpretation (written as $\mathcal{I}$ ) consists of a domain (written as $\Delta^{\mathcal{I}}$ ) and an interpretation function (written as ${ }^{\mathcal{I}}$ ), where the domain is a nonempty set of objects and the interpretation function maps each individual name $\mathrm{a} \in \mathbf{I}$ to an element $\mathrm{a}^{\mathcal{I}} \in \Delta^{\mathcal{I}}$, each concept name $\mathrm{CN} \in \mathbf{C}$ to a subset $\mathrm{CN}^{\mathcal{I}} \subseteq \Delta^{\mathcal{I}}$, and each role name $R N \in \mathbf{R}$ to a binary relation $R N^{\mathcal{I}} \subseteq \Delta^{\mathcal{I}} \times \Delta^{\mathcal{I}}$. The interpretation function can be extended to give semantics to concept and role descriptions. These are given in Table 1.

A $\mathcal{S H} \mathcal{H} \mathcal{N}$ knowledge base (KB) consists of a TBox, an RBox and an ABox. A $\mathcal{S H \mathcal { N } N}$ TBox is a finite set of concept inclusion axioms of the form $C \sqsubseteq D$, or concept equivalence

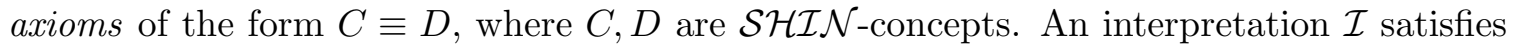
$C \sqsubseteq D$ if $C^{\mathcal{I}} \subseteq D^{\mathcal{I}}$ and it satisfies $C \equiv D$ if $C^{\mathcal{I}}=D^{\mathcal{I}}$. Note that concept inclusion axioms of the above form are called general concept inclusions (Horrocks \& Sattler, 1999; Baader, 1990). A $\mathcal{S H \mathcal { I N }} R B$ ox is a finite set of transitive role axioms (Trans $(R)$ ), and role inclusion axioms $(R \sqsubseteq S)$. An interpretation $\mathcal{I}$ satisfies $\operatorname{Trans}(R)$ if, for all $x, y, z \in \Delta^{\mathcal{I}}$, $\{\langle x, y\rangle,\langle y, z\rangle\} \subseteq R^{\mathcal{I}} \rightarrow\langle x, z\rangle \in R^{\mathcal{I}}$, and it satisfies $R \sqsubseteq S$ if $R^{\mathcal{I}} \subseteq S^{\mathcal{I}}$. A set of role inclusion axioms defines a role hierarchy. For a role hierarchy we introduce $\stackrel{\Xi}{=}$ as the transitive-reflexive closure of $\sqsubseteq$. At last, observe that if $R \sqsubseteq S$, then the semantics of role inclusion axioms imply that $\operatorname{lnv}(R)^{\mathcal{I}} \subseteq \operatorname{lnv}(S)^{\mathcal{I}}$, while the semantics of inverse roles imply that $\operatorname{Trans}(\operatorname{Inv}(R))$. A $\mathcal{S I}$ RBox is obtained by a $\mathcal{S H \mathcal { I N }}$ RBox if we disallow role inclusion axioms. A $\mathcal{S H \mathcal { N } N}$ ABox is a finite set of individual axioms (or assertions) of the form $\mathrm{a}: C$, called concept assertions, or $\langle\mathrm{a}, \mathrm{b}\rangle: R$, called role assertions, or of the form $\mathrm{a} \neq \mathrm{b}$.

2. A role is called simple if it is neither transitive nor has any transitive sub-roles. This is crucial in order to get a decidable logic (Horrocks, Sattler, \& Tobies, 1999). 
An interpretation $\mathcal{I}$ satisfies a $: C$ if $\mathrm{a}^{\mathcal{I}} \in C^{\mathcal{I}}$, it satisfies $\langle\mathrm{a}, \mathrm{b}\rangle: R$ if $\left\langle\mathrm{a}^{\mathcal{I}}, \mathrm{b}^{\mathcal{I}}\right\rangle \in R^{\mathcal{I}}$, and it satisfies $\mathrm{a} \neq \mathrm{b}$ if $\mathrm{a}^{\mathcal{I}} \neq \mathrm{b}^{\mathcal{I}}$. A $\mathcal{S} \mathcal{I}$ ABox is obtained by a $\mathcal{S H \mathcal { I N }}$ ABox by disallowing inequality axioms $\mathrm{a} \neq \mathrm{b}$. An interpretation $\mathcal{I}$ satisfies a $\mathcal{S H \mathcal { N } N}$ knowledge base $\Sigma$ if it satisfies all the axioms in $\Sigma$. $\Sigma$ is satisfiable (unsatisfiable) iff there exists (does not exist)

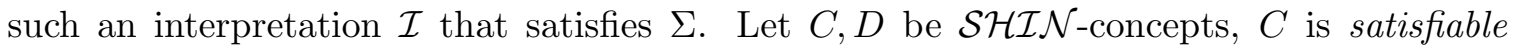
(unsatisfiable) w.r.t. $\Sigma$ iff there exists (does not exist) an interpretation $\mathcal{I}$ of $\Sigma$ s.t. $C^{\mathcal{I}} \neq \emptyset$; $C$ subsumes $D$ w.r.t. $\Sigma$ iff for every interpretation $\mathcal{I}$ of $\Sigma$ we have $C^{\mathcal{I}} \subseteq D^{\mathcal{I}}$. Given a concept axiom, a role axiom, or an assertion $\Psi, \Sigma$ entails $\Psi$, written as $\Sigma \models \Psi$, iff for all models $\mathcal{I}$ of $\Sigma$ we have $\mathcal{I}$ satisfies $\Psi$.

\subsection{Fuzzy Sets}

Fuzzy set theory and fuzzy logic are widely used today for capturing the inherent vagueness (the lack of distinct boundaries of sets) that exists in real life applications (Klir \& Yuan, 1995). The notion of a fuzzy set was first introduced by Zadeh (1965). While in classical set theory an element either belongs to a set or not, in fuzzy set theory elements belong only to a certain degree. More formally, let $X$ be a collection of elements (called universe of discourse) i.e $X=\left\{x_{1}, x_{2}, \ldots\right\}$. A crisp subset $A$ of $X$ is any collection of elements of $X$ that can be defined with the aid of its characteristic function $\chi_{A}(x)$ that assigns any $x \in X$ to a value 1 or 0 if this element belongs to $X$ or not, respectively. On the other hand, a fuzzy subset $A$ of $X$, is defined by a membership function $\mu_{A}(x)$, or simply $A(x)$, for each $x \in X$. This membership function assigns any $x \in X$ to a value between 0 and 1 that represents the degree in which this element belongs to $A$. Additionally, a fuzzy binary relation $R$ over two crisp sets $X$ and $Y$ is a function $R: X \times Y \rightarrow[0,1]$. For example, one can say that Tom belongs to the set of Tall people to a degree of 0.8 , writing $\operatorname{Tall}(\operatorname{Tom})=0.8$, or that the object $o_{1}$ is part of the object $o_{2}$ to a degree of 0.6, writing isPartOf $\left(o_{1}, o_{2}\right)=0.6$. Several properties of fuzzy binary relations have been investigated in the literature (Klir \& Yuan, 1995). For example, a binary fuzzy relation is called sup-min transitive if $R(x, z) \geq \sup _{y \in Y}\{\min (R(x, y), R(y, z))\}$, while the inverse of a relation $R$ is defined as $R^{-1}(y, x)=R(x, y)$ (Klir \& Yuan, 1995).

Using the above idea, the most important operations defined on crisp sets and relations, like the boolean operations (complement, union, and intersection etc.), are extended in order to cover fuzzy sets and fuzzy relations. Accordingly, a sound and complete mathematical framework that plays an important role in the management of imprecise and vague information has been defined and used in a wide set of scientific areas including expert systems and decision making (Zimmermann, 1987), pattern recognition (Kandel, 1982), image analysis and computer vision (Krishnapuram \& Keller, 1992), medicine (Oguntade \& Beaumont, 1982), control (Sugeno, 1985), etc.

\subsection{Fuzzy Set Theoretic Operations}

In this section, we will explain how to extend boolean operations and logical implications in the context of fuzzy sets and fuzzy logics. These operations are now performed by mathematical functions over the unit interval.

The operation of complement is performed by a unary operation, $c:[0,1] \rightarrow[0,1]$, called fuzzy complement. In order to provide meaningful fuzzy complements, such functions should 
satisfy certain properties. More precisely, they should satisfy the boundary conditions, $c(0)=1$ and $c(1)=0$, and be monotonic decreasing, for $a \leq b, c(a) \geq c(b)$. In the current paper we will use the Lukasiewicz negation, $c(a)=1-a$, which additionally is continuous and involutive, for each $a \in[0,1], c(c(a))=a$ holds. In the cases of fuzzy intersection and fuzzy union the mathematical functions used are binary over the unit interval. These functions are usually called norm operations referred to as t-norms $(t)$, in the case of fuzzy intersection, and t-conorms (or s-norms) $(u)$, in the case of fuzzy union (Klement, Mesiar, \& Pap, 2004). Again these operations should satisfy certain mathematical properties. More precisely, a t-norm (t-conorm) satisfies the boundary condition, $t(a, 1)=a$ $(u(a, 0)=a)$, is monotonic increasing, for $b \leq d$ then $t(a, b) \leq t(a, d)(u(a, b) \leq u(a, d))$, commutative, $t(a, b)=t(b, a)(u(a, b)=u(b, a))$, and associative, $t(a, t(b, c))=t(t(a, b), c)$ $(u(a, u(b, c))=u(u(a, b), c))$. Though there is a wealth of such operations in the literature (Klir \& Yuan, 1995) we restrict our attention to specific ones. More precisely, we are using the Gödel t-norm, $t(a, b)=\min (a, b)$ and the Gödel t-conorm, $u(a, b)=\max (a, b)$. Additionally to the aforementioned properties, these operations are also idempotent, i.e. $\min (a, a)=a$ and $\max (a, a)=a$, hold. Finally, a fuzzy implication is performed by a binary operation, of the form $\mathcal{J}:[0,1] \times[0,1] \rightarrow[0,1]$. In the current paper we use the Kleene-Dienes fuzzy implication which is provided by the equation, $\mathcal{J}(a, b)=\max (c(a), b)$. The reason for restricting our attention to these operations would be made clear in section 5.1. We now recall a property of the max norm operation that we are going to use in the investigation of the properties of transitive relations under the framework of fuzzy set theory.

Lemma 2.2 (Hajek, 1998) For any $a, b \in[0,1]$, where $j$ takes values from the index set $J$, the max operation satisfies the following property:

- $\inf _{j \in J} \max \left(a, b_{j}\right)=\max \left(a, \inf _{j \in J} b_{j}\right)$.

\section{The $\mathbf{f}_{K D}-\mathcal{S H} \mathcal{H} \mathcal{N}$ DL}

In this section, we introduce a fuzzy extension of the $\mathcal{S H \mathcal { N } N}$ DL presented in Section 2.1. Following Stoilos, Stamou, Tzouvaras, Pan, and Horrocks (2005b), since we are using the Kleene-Dienes (KD) fuzzy implication in our language, we call it $\mathrm{f}_{K D^{-}} \mathcal{S} \mathcal{H} \mathcal{I N}$. This presentation follows the standard syntax and semantics of fuzzy DLs, that has been introduced in the literature (Straccia, 2001; Hölldobler et al., 2002; Sánchez \& Tettamanzi, 2004). More precisely, $\mathrm{f}_{K D}-\mathcal{S H \mathcal { I N }}$ was first presented by Straccia $(2005 \mathrm{~b})$. For completeness reasons

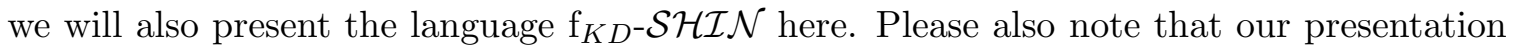
differs from that of Straccia (2005b) in the semantics of concept and role inclusion axioms.

As usual, we consider an alphabet of distinct concept names $(\mathbf{C})$, role names $(\mathbf{R})$ and

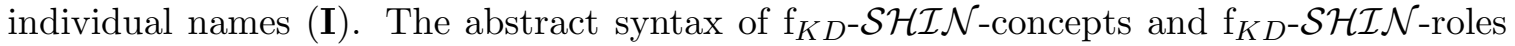
(and respectively of $\mathrm{f}_{K D^{-}} \mathcal{S} \mathcal{I}$-concepts and $\mathrm{f}_{K D^{-}} \mathcal{S} \mathcal{I}$-roles) is the same as their $\mathcal{S H \mathcal { H } N}$ counterparts; however, their semantics is based on fuzzy interpretations (see below). Similarly, $\mathrm{f}_{K D^{-}} \mathcal{S H \mathcal { H } N}$ keeps the same syntax of concept and role axioms as their counterparts in $\mathcal{S H \mathcal { I N }}$. Interestingly, $\mathrm{f}_{K D^{-}} \mathcal{S} \mathcal{H} \mathcal{I} \mathcal{N}$ extends $\mathcal{S H \mathcal { I N }}$ individual axioms (assertions) into fuzzy individual axioms, or fuzzy assertions (following, Straccia, 2001), where membership degrees can be asserted. 
Firstly, by using membership functions that range over the interval $[0,1]$, classical interpretations can be extended to the concept of fuzzy interpretations (Straccia, 2001). Here we abuse the symbols and define a fuzzy interpretation as a pair $\mathcal{I}=\left(\Delta^{\mathcal{I}}, \cdot^{\mathcal{I}}\right),{ }^{3}$ where the domain $\Delta^{\mathcal{I}}$ is a non-empty set of objects and ${ }^{\mathcal{I}}$ is a fuzzy interpretation function, which maps

1. an individual name $\mathrm{a} \in \mathbf{I}$ to an element $\mathbf{a}^{\mathcal{I}} \in \Delta^{\mathcal{I}}$,

2. a concept name $\mathbf{A} \in \mathbf{C}$ to a membership function $A^{\mathcal{I}}: \Delta^{\mathcal{I}} \rightarrow[0,1]$,

3. a role name $R \in \mathbf{R}$ to a membership function $R^{\mathcal{I}}: \Delta^{\mathcal{I}} \times \Delta^{\mathcal{I}} \rightarrow[0,1]$.

For example, if $o \in \Delta^{\mathcal{I}}$ then $A^{\mathcal{I}}(o)$ gives the degree that the object $o$ belongs to the fuzzy concept $A$, e.g. $A^{\mathcal{I}}(o)=0.8$. By using the fuzzy set theoretic operations defined in section 2.3 , the fuzzy interpretation function can be extended to give semantics to $\mathrm{f}_{K D^{-}} \mathcal{S H \mathcal { H } N}$ concepts and $\mathrm{f}_{K D^{-}} \mathcal{S} \mathcal{H} \mathcal{I} \mathcal{N}$-roles. For example, since we use the max function for fuzzy union the membership degree of an object $a$ to the fuzzy concept $(C \sqcup D)^{\mathcal{I}}$ is equal to $\max \left(C^{\mathcal{I}}(a), D^{\mathcal{I}}(a)\right)$. Moreover since, according to Table 1 , a value restriction $\forall R$. $C$ is an implication of the form, $\forall y(R(x, y) \rightarrow C(y))$, we can interpret $\forall$ as inf (Hajek, 1998), and $\rightarrow$ as the Kleene-Dienes fuzzy implication and finally have the equation, $\inf _{b \in \Delta^{\mathcal{I}}}\{\max (1-$ $\left.\left.R^{\mathcal{I}}(a, b), C^{\mathcal{I}}(b)\right)\right\}$. The complete set of semantics is depicted in Table 2. We have to note that there are many proposals for semantics of number restrictions in fuzzy DLs (Sánchez \& Tettamanzi, 2004; Straccia, 2005b). We choose to follow the semantics proposed by Straccia (2005b) since they are based in the First-Order interpretation of number restrictions (Baader et al., 2002a). Moreover, as it is shown by Stoilos, Stamou, Tzouvaras, Pan, and Horrocks (2005a) and as we will see in section 6, under these semantics all inference services of $\mathrm{f}_{K D^{-}} \mathcal{S H \mathcal { H } \mathcal { N }}$ stay decidable and reasoning can be reduced to a simple counting problem, yielding an efficient algorithm. Note that, although most of the above semantics have been presented elsewhere (Sánchez \& Tettamanzi, 2004; Straccia, 2005b), we include them here simply for the sake of completeness.

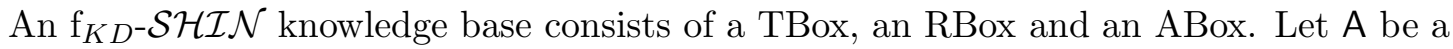
concept name and $C$ an $\mathrm{f}_{K D^{-}} \mathcal{S H \mathcal { I N }}$ concept. An $\mathrm{f}_{K D^{-}} \mathcal{S H \mathcal { I N }}$ TBox is a finite set of fuzzy concept axioms of the form $\mathrm{A} \sqsubseteq C$, called fuzzy inclusion introductions, and of the form $\mathrm{A} \equiv C$, called fuzzy equivalence introductions. A fuzzy interpretation $\mathcal{I}$ satisfies $\mathrm{A} \sqsubseteq C$ if $\forall o \in \Delta^{\mathcal{I}}, \mathrm{A}^{\mathcal{I}}(o) \leq C^{\mathcal{I}}(o)$. A fuzzy interpretation satisfies $\mathrm{A} \equiv C$ if $\forall o \in \Delta^{\mathcal{I}}, \mathrm{A}^{\mathcal{I}}(o)=C^{\mathcal{I}}(o)$. A fuzzy interpretation $\mathcal{I}$ satisfies an $\mathrm{f}_{K D}-\mathcal{S H \mathcal { H } \mathcal { N }}$ TBox $\mathcal{T}$ iff it satisfies all fuzzy concept axioms in $\mathcal{T}$; in this case, we say that $\mathcal{I}$ is a model of $\mathcal{T}$.

There are two remarks here. Firstly, we give a crisp subsumption of fuzzy concepts here, which is the usual way subsumption is defined in the context of fuzzy sets (Klir \& Yuan, 1995). In contrast, Straccia (2005b) defines a fuzzy subsumption of fuzzy concepts. As it was noted by Bobillo, Delgado, and Gómez-Romero (2006) in $\mathrm{f}_{K D}$-DLs fuzzy subsumption is sometimes counterintuitive. Secondly, as we can see, we are only allowing for simple TBoxes. A TBox $\mathcal{T}$ is called simple if it neither includes cyclic nor general concept inclusions, i.e. axioms are of the form $A \sqsubseteq C$ or $A \equiv C$, where $A$ is a concept name that is never defined by

3. In the rest of the paper, we use $\mathcal{I}=\left(\Delta^{\mathcal{I}},{ }^{\mathcal{I}}\right)$ to represent fuzzy interpretations instead of crisp interpretations. 


\begin{tabular}{|c|c|c|}
\hline Constructor & Syntax & Semantics \\
\hline top & $\bar{\top}$ & $\bar{\top}^{\mathcal{I}}(a)=1$ \\
\hline bottom & $\perp$ & $\perp^{\mathcal{I}}(a)=0$ \\
\hline general negation & $\neg C$ & $(\neg C)^{\mathcal{I}}(a)=1-C^{\mathcal{I}}(a)$ \\
\hline conjunction & $C \sqcap D$ & $(C \sqcap D)^{\mathcal{I}}(a)=\min \left(C^{\mathcal{I}}(a), D^{\mathcal{I}}(a)\right)$ \\
\hline disjunction & $C \sqcup D$ & $(C \sqcup D)^{\mathcal{I}}(a)=\max \left(C^{\mathcal{I}}(a), D^{\mathcal{I}}(a)\right)$ \\
\hline exists restriction & $\exists R . C$ & $(\exists R . C)^{\mathcal{I}}(a)=\sup _{b \in \Delta^{\mathcal{I}}}\left\{\min \left(R^{\mathcal{I}}(a, b), C^{\mathcal{I}}(b)\right)\right\}$ \\
\hline value restriction & $\forall R . C$ & $(\forall R . C)^{\mathcal{I}}(a)=\inf _{b \in \Delta^{\mathcal{I}}}\left\{\max \left(1-R^{\mathcal{I}}(a, b), C^{\mathcal{I}}(b)\right)\right\}$ \\
\hline at-most & $\leq p R$ & $\inf _{b_{1}, \ldots, b_{p+1} \in \Delta^{\mathcal{I}} \max _{i=1}^{p+1}\left\{1-R^{\mathcal{I}}\left(a, b_{i}\right)\right\}}$ \\
\hline at-least & $\geq p R$ & $\sup _{b_{1}, \ldots, b_{p} \in \Delta^{\mathcal{I}}} \min _{i=1}^{p}\left\{R^{\mathcal{I}}\left(a, b_{i}\right)\right\}$ \\
\hline inverse role & $R^{-}$ & $\left(R^{-}\right)^{\mathcal{I}}(b, a)=R^{\mathcal{I}}(a, b)$ \\
\hline
\end{tabular}

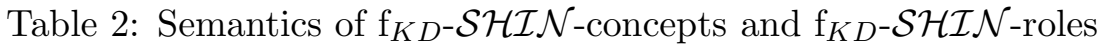

itself either directly or indirectly. A procedure to deal with cyclic and general TBoxes, in the context of fuzzy DLs, has been recently developed by Stoilos, Straccia, Stamou, and Pan (2006), while also in parallel a slightly different technique was presented by $\mathrm{Li}, \mathrm{Xu}, \mathrm{Lu}$, and Kang (2006a). This process involves additional expansion rules and a preprocessing step called normalization, which are not affected by the expressivity of the underlying fuzzy DL. Hence, in order to keep our presentation simple we will not consider general TBoxes in the following, but we will focus on the decidability and reasoning of $\mathrm{f}_{K D^{-}} \mathcal{S} \mathcal{I}$ and $\mathrm{f}_{K D^{-}} \mathcal{S H \mathcal { I N }}$, which involve many technical details. At the end of section 6 we will comment more on the issue of handling GCIs in the $\mathrm{f}_{K D^{-}} \mathcal{S H \mathcal { I N }}$ language.

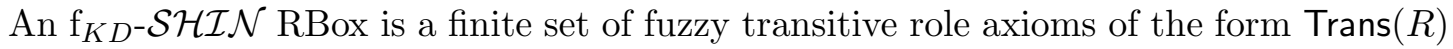

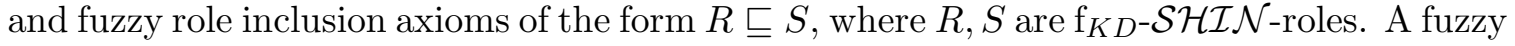
interpretation $\mathcal{I}$ satisfies $\operatorname{Trans}(R)$ if $\forall a, c \in \Delta^{\mathcal{I}}, R^{\mathcal{I}}(a, c) \geq \sup _{b \in \Delta^{\mathcal{I}}}\left\{\min \left(R^{\mathcal{I}}(a, b), R^{\mathcal{I}}(b, c)\right)\right\}$, while it satisfies $R \sqsubseteq S$ if $\forall a, b \in \Delta^{\mathcal{I}}, R^{\mathcal{I}}(a, b) \leq S^{\mathcal{I}}(a, b)$. Note that the semantics result from the definition of sup-min transitive relations in fuzzy set theory. A fuzzy interpretation $\mathcal{I}$ satisfies an $\mathrm{f}_{K D^{-}} \mathcal{S} \mathcal{H} \mathcal{I} \mathcal{N}$ RBox $\mathcal{R}$ iff it satisfies all fuzzy transitive role axioms in $\mathcal{R}$; in this case, we say that $\mathcal{I}$ is a model of $\mathcal{R}$. Similarly with the classical $\mathcal{S H \mathcal { I N }}$ language, the semantics of inverse roles and role inclusion axioms of $\mathrm{f}_{K D}-\mathcal{S H \mathcal { I } N}$ imply that from $\operatorname{Trans}(R)$ and $R \sqsubseteq S$ it holds that $\operatorname{Trans}(\operatorname{Inv}(R))$ and $\operatorname{Inv}(R)^{-} \sqsubseteq \operatorname{lnv}(S)^{-}$.

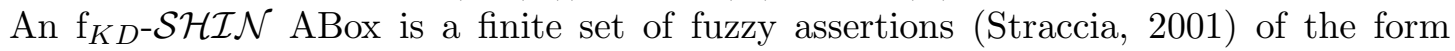
$(\mathrm{a}: C) \bowtie n$ or $(\langle\mathrm{a}, \mathrm{b}\rangle: R) \bowtie n$, where $\bowtie$ stands for $\geq,>, \leq$ and $<$, and $n \in[0,1]$ or of the form $\mathrm{a} \neq \mathrm{b}$. Intuitively, a fuzzy assertion of the form $(\mathrm{a}: C) \geq n$ means that the membership degree of the individual a to the concept $C$ is at least equal to $n$. We call assertions defined by $\geq,>$ positive assertions, while those defined by $\leq,<$ negative assertions. Formally, given a fuzzy interpretation $\mathcal{I}$,

$$
\begin{array}{rll}
\mathcal{I} \text { satisfies }(\mathrm{a}: C) \geq n & \text { if } & C^{\mathcal{I}}\left(\mathrm{a}^{\mathcal{I}}\right) \geq n, \\
\mathcal{I} \text { satisfies }(\mathrm{a}: C) \leq n & \text { if } & C^{\mathcal{I}}\left(\mathrm{a}^{\mathcal{I}}\right) \leq n, \\
\mathcal{I} \text { satisfies }(\langle\mathrm{a}, \mathrm{b}\rangle: R) \geq n & \text { if } & R^{\mathcal{I}}\left(\mathrm{a}^{\mathcal{I}}, \mathrm{b}^{\mathcal{I}}\right) \geq n, \\
\mathcal{I} \text { satisfies }(\langle\mathrm{a}, \mathrm{b}\rangle: R) \leq n & \text { if } & R^{\mathcal{I}}\left(\mathrm{a}^{\mathcal{I}}, \mathrm{b}^{\mathcal{I}}\right) \leq n, \\
\mathcal{I} \text { satisfies } \mathrm{a} \neq \mathrm{b} & \text { if } & \mathrm{a}^{\mathcal{I}} \neq \mathrm{b}^{\mathcal{I}} .
\end{array}
$$


The satisfiability of fuzzy assertions with $>,<$ is defined analogously. Observe that, we can also simulate assertions of the form $(\mathrm{a}: C)=n$ by considering two assertions of the form (a :C) $\geq n$ and (a :C) $\leq n$ (Hölldobler et al., 2002; Straccia, 2001). A fuzzy interpretation $\mathcal{I}$ satisfies an $\mathrm{f}_{K D^{-}} \mathcal{S} \mathcal{H} \mathcal{I} \mathcal{N}$ ABox $\mathcal{A}$ iff it satisfies all fuzzy assertions in $\mathcal{A}$; in this case, we say that $\mathcal{I}$ is a model of $\mathcal{A}$.

Furthermore, as it was noted by Straccia (2001, 2005b), due to the mathematical properties of the norm operations defined in section 2.3 , the following $\mathrm{f}_{K D^{-}} \mathcal{S} \mathcal{H} \mathcal{I} \mathcal{N}$-concept equivalences are satisfied: $\neg \top \equiv \perp, \neg \perp \equiv \top, C \sqcap \top \equiv C, C \sqcup \perp \equiv C, C \sqcup \top \equiv \top$ and $C \sqcap \perp \equiv \perp$. Furthermore, since the Lukasiewicz complement is involutive it holds that, $\neg \neg C \equiv C$. Moreover, the De Morgan laws: $C_{1} \sqcap C_{2} \equiv \neg\left(\neg C_{1} \sqcup \neg C_{2}\right), C_{1} \sqcup C_{2} \equiv \neg\left(\neg C_{1} \sqcap \neg C_{2}\right)$, are satisfied. As a consequence of the satisfiability of the De Morgan laws and the use of the Kleene-Dienes fuzzy implication the following concept equivalences also hold.

$$
\begin{aligned}
& \neg \exists R . C \equiv \forall R .(\neg C), \quad \neg \forall R . C \equiv \exists R .(\neg C), \\
& \neg \leq p_{1} R \equiv \geq\left(p_{1}+1\right) R, \quad \neg \geq p_{1} R \equiv \begin{cases}\leq\left(p_{1}-1\right) R, & p_{1} \in \mathbb{N}^{*} \\
\perp, & p_{1}=0\end{cases}
\end{aligned}
$$

At last note that the classical laws of contradiction $(C \sqcap \neg C \equiv \perp)$ and excluded middle $(C \sqcup \neg C \equiv \top)$, do not hold.

Example 3.1 Let us revisit the fuzzy knowledge base $(\Sigma)$ that we informally introduced in section 1. Formally, the knowledge base can be defined as follows: $\Sigma=\langle\mathcal{T}, \mathcal{R}, \mathcal{A}\rangle$, where

$$
\begin{aligned}
\mathcal{T}= & \{\text { Arm } \sqsubseteq \text { تisPartOf.Body }, \\
& \text { Body } \sqsubseteq \text { isPartOf.Human }\} \\
\mathcal{A}= & \left\{\left(\left\langle\mathrm{o}_{1}, \mathrm{o}_{2}\right\rangle: \text { isPartOf }\right) \geq 0.8,\left(\left\langle\mathrm{o}_{2}, \mathrm{o}_{3}\right\rangle: \text { isPartOf }\right) \geq 0.9,\right. \\
& \left.\left(\mathrm{o}_{2}: \text { Body }\right) \geq 0.85,\left(\mathrm{o}_{1}: \text { Arm }\right) \geq 0.75\right\} \\
\mathcal{R}= & \{\text { Trans(isPartOf })\} .
\end{aligned}
$$

Now, in order for some fuzzy interpretation $\mathcal{I}$ to be a model of $\mathcal{T}$ it should hold that $\operatorname{Arm}^{\mathcal{I}}\left(\mathrm{o}_{i}^{\mathcal{I}}\right) \leq(\exists \text { isPartOf.Body })^{\mathcal{I}}\left(\mathrm{o}_{i}^{\mathcal{I}}\right)$ and $\operatorname{Body}^{\mathcal{I}}\left(\mathrm{o}_{i}^{\mathcal{I}}\right) \leq(\exists \text { isPartOf.Body })^{\mathcal{I}}\left(\mathrm{o}_{i}^{\mathcal{I}}\right), \forall \mathrm{o}_{i}^{\mathcal{I}} \in \Delta^{\mathcal{I}}$. Furthermore, if isPartOf ${ }^{\mathcal{I}}\left(\mathrm{o}_{1}^{\mathcal{I}}, \mathrm{o}_{2}^{\mathcal{I}}\right) \geq 0.8$, isPartOf ${ }^{\mathcal{I}}\left(\mathrm{o}_{2}^{\mathcal{I}}, \mathrm{o}_{3}^{\mathcal{I}}\right) \geq 0.9$, $\operatorname{Body}^{\mathcal{I}}\left(\mathrm{o}_{2}^{\mathcal{I}}\right) \geq 0.85$ and $\operatorname{Arm}^{\mathcal{I}}\left(\mathrm{o}_{1}^{\mathcal{I}}\right) \geq 0.75$, then $\mathcal{I}$ is also a model of $\mathcal{A}$. As a model of the RBox $\mathcal{R}, \mathcal{I}$ should also satisfy that isPartOf ${ }^{\mathcal{I}}\left(\mathrm{o}_{1}^{\mathcal{I}}, \mathrm{o}_{3}^{\mathcal{I}}\right) \geq \sup _{a \in \Delta^{\mathcal{I}}}\left\{\min \left(\right.\right.$ isPartOf $^{\mathcal{I}}\left(\mathrm{o}_{1}^{\mathcal{I}}, a\right)$, isPartOf $\left.\left.\operatorname{Is}^{\mathcal{I}}\left(a, \mathrm{o}_{3}^{\mathcal{I}}\right)\right)\right\}=$ $\sup \{\ldots, \min (0.8,0.9), \ldots\} \geq 0.8$.

Now let us consider the concept $\exists$ hasPart.Body $\sqcap \exists$ hasPart.Arm that we mentioned in Section 1. Due to the semantics of existential restrictions presented in Table 2, we have that

$$
\begin{aligned}
& (\exists \text { hasPart.Body })^{\mathcal{I}}\left(\mathrm{o}^{\mathcal{I}}\right)=\sup _{a \in \Delta^{\mathcal{I}}}\left\{\min \left(\left(\text { isPartOf }^{-}\right)^{\mathcal{I}}\left(\mathrm{o}_{3}^{\mathcal{I}}, a\right), \operatorname{Body}^{\mathcal{I}}(a)\right)\right\} \geq 0.85 \\
& (\exists \text { hasPart.Arm })^{\mathcal{I}}\left(\mathrm{o}_{3}^{\mathcal{I}}\right)=\sup _{a \in \Delta^{\mathcal{I}}}\left\{\min \left(\left(\text { isPartOf }^{-}\right)^{\mathcal{I}}\left(\mathrm{o}_{3}^{\mathcal{I}}, a\right), \operatorname{Arm}^{\mathcal{I}}(a)\right)\right\} \geq 0.75 .
\end{aligned}
$$

Hence $\mathrm{o}_{3}$ would belong in the intersection of the two concepts with the minimum membership degree which is greater or equal than 0.75 , as we claimed in Section 1. 


\begin{tabular}{|l|l|l|}
\hline & $\phi<m$ & $\phi \leq m$ \\
\hline$\phi \geq n$ & $n \geq m$ & $n>m$ \\
\hline$\phi>n$ & $n \geq m$ & $n \geq m$ \\
\hline
\end{tabular}

Table 3: Conjugated pairs of fuzzy assertions

Following Straccia (2001), we introduce the concept of conjugated pairs of fuzzy assertions to represent pairs of assertions that form a contradiction. The possible conjugated pairs are defined in Table 3, where $\phi$ represents a $\mathcal{S I}$ assertion. For example, if $\phi=\mathrm{a}: C$, then the assertions $(\mathrm{a}: C) \geq 0.7$ and $(\mathrm{a}: C)<0.7$ conjugate. Furthermore, due to the presence of inverse roles and role inclusion axioms the definition should be slightly extended from that of Straccia (2001) and hence, one should also take under consideration possible inverse roles or a role hierarchy when checking for conjugation two role assertions. For example, if $R \stackrel{\Xi}{=} S$, then the assertion $(\langle\mathrm{a}, \mathrm{b}\rangle: R) \geq 0.9$, conjugates with $(\langle\mathrm{b}, \mathrm{a}\rangle: \operatorname{lnv}(S)) \leq 0.4$; similarly for the rest of the inequalities.

Now, we will define the reasoning problems of the $\mathrm{f}_{K D}-\mathcal{S H \mathcal { H } N} \mathrm{DL}$.

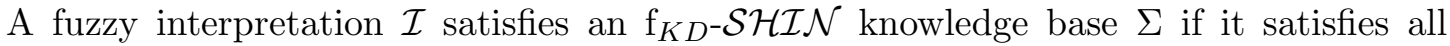

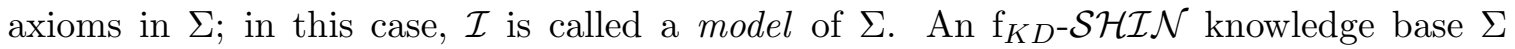
is satisfiable (unsatisfiable) iff there exists (does not exist) a fuzzy interpretation $\mathcal{I}$ which

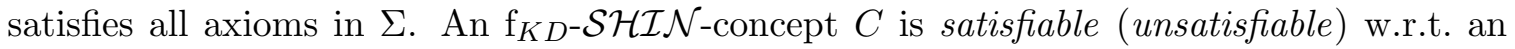
RBox $\mathcal{R}$ and a TBox $\mathcal{T}$ iff there exists (does not exist) some model $\mathcal{I}$ of $\mathcal{R}$ and $\mathcal{T}$ for which there is some $a \in \Delta^{\mathcal{I}}$ such that $C^{\mathcal{I}}(a)=n$, and $n \in(0,1]$. In this case, $C$ is called

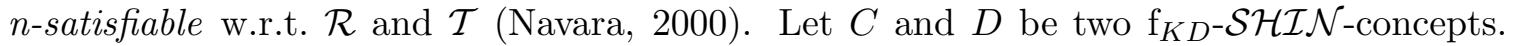
We say that $C$ is subsumed by $D$ w.r.t. $\mathcal{R}$ and $\mathcal{T}$ if for every model $\mathcal{I}$ of $\mathcal{R}$ and $\mathcal{T}$ it holds that, $\forall x \in \Delta^{\mathcal{I}}$. $C^{\mathcal{I}}(x) \leq D^{\mathcal{I}}(x)$. Furthermore, an $\mathrm{f}_{K D^{-}} \mathcal{S} \mathcal{H} \mathcal{I} \mathcal{N}$ ABox $\mathcal{A}$ is consistent w.r.t. $\mathcal{R}$ and $\mathcal{T}$ if there exists a model $\mathcal{I}$ of $\mathcal{R}$ and $\mathcal{T}$ that that is also a model of $\mathcal{A}$. Moreover, given a fuzzy concept axiom or a fuzzy assertion $\Psi \in\{C \sqsubseteq D, C \equiv D, \phi \bowtie n\}$, an $\mathrm{f}_{K D^{-}} \mathcal{S} \mathcal{H} \mathcal{I N}$ knowledge base $\Sigma$ entails $\Psi$, written $\Sigma \models \Psi$, iff all models of $\Sigma$ also satisfy $\Psi$.

Furthermore, by studying Table 3 , we can conclude that an $\mathrm{f}_{K D^{-}} \mathcal{S} \mathcal{H} \mathcal{I N}$ ABox $\mathcal{A}$ can contain a number of positive or negative assertions without forming a contradiction. Therefore, it is useful to compute lower and upper bounds of truth-values. Given an

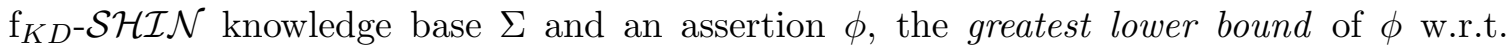
$\Sigma$ is $\operatorname{glb}(\Sigma, \phi)=\sup \{n: \Sigma \mid=\phi \geq n\}$, where $\sup \emptyset=0$. Similarly, the least upper bound of $\phi$ w.r.t. $\Sigma$ is $\operatorname{lub}(\Sigma, \phi)=\inf \{n: \Sigma \models \phi \leq n\}$, where $\inf \emptyset=1$. A decision procedure to solve the best truth-value bound was provided by Straccia (2001). In that procedure the membership degrees that appear in a $\mathrm{f}_{K D^{-}} \mathcal{S} \mathcal{H} \mathcal{I} \mathcal{N}$ ABox, together with their complemented values and the degrees $0,0.5$ and 1, were collected in a set of membership degrees $N^{\Sigma}$ and subsequently the entailment of a fuzzy assertions $\phi \geq n$ and $\phi \leq n$, for all $n \in N^{\Sigma}$ was tested, thus determining glb and lub. Obviously this procedure is independent of the expressivity of the DL language, and thus also applicable in our context.

Remark 3.2 From Table 2 we see that the semantics of the value and existential restrictions in fuzzy DLs are defined with the aid of an infimum and a supremum operation. This means that we can construct an infinite interpretation $\mathcal{I}$, i.e. an interpretation 
where $\Delta^{\mathcal{I}}=\left\{b_{1}, b_{2}, \ldots\right\}$ contains infinite number of objects, for which $\forall R$.C is n-satisfiable $\left((\forall R . C)^{\mathcal{I}}(a)=n\right.$ for some $\left.a \in \Delta^{\mathcal{I}}\right)$ but for all $b_{i} \in \Delta^{\mathcal{I}}, \max \left(1-R^{\mathcal{I}}\left(a, b_{i}\right), C^{\mathcal{I}}\left(b_{i}\right)\right)>n$. This is possible since although the maximum of the membership degrees involved for each individual object $b_{i}$ is strictly greater than $n$ the limit of the infinite sequence could converge to $n$. This fact was first noted for fuzzy DLs by Hajek (2005), introducing the notion of witnessed model for fuzzy DLs. A model is called witnessed if for $(\forall R . C)^{\mathcal{I}}(a)=n$ there is some $b \in \Delta^{\mathcal{I}}$ such that either $R^{\mathcal{I}}\left(a, b_{i}\right)=1-n$ or $C^{\mathcal{I}}\left(b_{i}\right)=n$, i.e. there is some $b \in \Delta^{\mathcal{I}}$ that witnesses the membership degree of a to $\forall R$.C. Fortunately, there are fuzzy logics that have an infinite model if and only if they have a witnessed model. More precisely, Hajek proves this property for the Lukasiewicz fuzzy logic ${ }^{4}$. He then concludes that the same proofs can be modified to apply to the fuzzy logic defined by the fuzzy operators we are using in the current paper. That is because these operators are definable in the Lukasiewicz logic (Mostert 85 Shields, 1957). For the rest of the paper, without loss of generality, we are going to consider only witnessed models.

In this paper, we will provide an algorithm to decide the fuzzy ABox consistency problem w.r.t. an RBox in very expressive fuzzy DLs. Many other reasoning problems can be reduced to this problem. Firstly, concept satisfiability for a fuzzy concept $C$ can be reduced to consistency checking of the fuzzy ABox $\{(\mathrm{a}: C)>0\}$. Secondly, in this paper, we only consider unfoldable TBoxes, where KB satisfiability can be reduced to ABox consistency w.r.t. an RBox. A TBox is unfoldable if it contains no cycles and contains only unique introductions, i.e., concept axioms with only concept names appearing on the left hand side and, for each concept name $A$, there is at most one axiom in $\mathcal{T}$ of which A appears on the left side. A knowledge base with an unfoldable TBox can be transformed into an equivalent one with an empty TBox by a transformation called unfolding, or expansion (Nebel, 1990): Concept inclusion introductions $\mathrm{A} \sqsubseteq C$ are replaced by concept equivalence introductions $\mathrm{A} \equiv \mathrm{A}^{\prime} \sqcap C$, where $\mathrm{A}^{\prime}$ is a new concept name, which stands for the qualities that distinguish the elements of $\mathrm{A}$ from the other elements of $C$. Subsequently, if $C$ is a complex concept expression, which is defined in terms of concept names, defined in the TBox, we replace their definitions in $C$. It can be proved that the initial TBox with the expanded one are equivalent.

Moreover, the problem of entailment can be reduced to the problem of fuzzy knowledge base satisfiability (Straccia, 2001). More precisely, for $\Sigma=\langle\mathcal{T}, \mathcal{R}, \mathcal{A}\rangle, \Sigma \models \phi \bowtie n$ iff $\Sigma^{\prime}=\langle\mathcal{T}, \mathcal{R}, \mathcal{A} \cup\{\phi \neg \bowtie n\}\rangle$ is unsatisfiable. With $\neg \bowtie$, we denote the "negation" of inequalities; e.g., if $\bowtie \equiv \geq$ then $\neg \bowtie \equiv<$, while if $\bowtie \equiv<$ then $\neg \bowtie \equiv \geq$. Finally, the subsumption problem of two fuzzy concepts $C$ and $D$ w.r.t. a TBox can also be reduced to the fuzzy knowledge base satisfiability problem. More formally, Straccia (2001), proved that $\langle\mathcal{T}, \emptyset, \mathcal{A}\rangle=C \sqsubseteq D$ iff $\langle\mathcal{T}, \emptyset,\{(\mathrm{a}: C) \geq n,(\mathrm{a}: D)<n\}\rangle$, for both $n \in\left\{n_{1}, n_{2}\right\}, n_{1} \in(0,0.5]$ and $n_{2} \in(0.5,1]$, is unsatisfiable. The above reduction can be extended in order for a fuzzy knowledge base to also include an RBox. Please note that, in crisp DLs, in order to check if a concept $C$ is subsumed by a concept $D$ we check for the unsatisfiability of the concept, $C \sqcap \neg D$. This reduction to unsatisfiability is not applicable to $f_{K D}$-DLs since the fuzzy operations that we use do not satisfy the laws of contradiction and excluded middle.

4. Lukasiewicz fuzzy logic uses the t-norm $t(a, b)=\max (0, a+b-1)$, the t-conorm $u(a, b)=\min (1, a+b)$, the Lukasiewicz complement and the fuzzy implication $\mathcal{J}(a, b)=\min (1,1-a+b)$ 
We conclude the section with an example.

Example 3.3 Consider again our sample knowledge base ( $\Sigma)$. By applying the transformation of unfolding, defined earlier, one would obtain the following expanded fuzzy TBox:

$$
\begin{aligned}
\mathcal{T}^{\prime}= & \left\{\text { Arm } \equiv \text { Arm }^{\prime} \sqcap \exists\right. \text { isPartOf.Body, } \\
& \text { Body } \left.\equiv \text { Body }^{\prime} \sqcap \exists \text { isPartOf.Human }\right\}
\end{aligned}
$$

while the respective fuzzy assertions would be transformed to

$$
\begin{aligned}
\mathcal{A}^{\prime}= & \left\{\left(\mathrm{o}_{2}: \text { Body }^{\prime} \sqcap \text { ヨisPartOf.Human }\right) \geq 0.85\right\rangle, \\
& \left.\left.\left(\mathrm{o}_{1}: \text { Arm }^{\prime} \sqcap \exists \text { isPartOf.Body }\right) \geq 0.75\right\rangle\right\}
\end{aligned}
$$

Now, let us formally specify the query we introduced in section 1. If $\Sigma^{\prime}=\left\langle\mathcal{T}^{\prime}, \mathcal{R}, \mathcal{A}^{\prime}\right\rangle$ is our modified knowledge base, after unfolding, the query would have the form $\Sigma^{\prime} \models\left(\mathrm{o}_{3}\right.$ : $\exists$ hasPart.Body $\sqcap \exists$ hasPart.Arm) $\geq 0.75$. According to our previous discussion in order to check for the entailment of such a query one should check for the consistency of the fuzzy $A B o x \mathcal{A}^{\prime} \cup\left\{\left(\mathrm{o}_{3}: \exists\right.\right.$ hasPart.Body $\sqcap \exists$ hasPart.Arm $\left.)<0.75\right\}$, w.r.t. the $R B$ ox $\mathcal{R}$, since after the expansion we can remove $\mathcal{T}^{\prime}$. Our task in the following sections is to provide a procedure that decides the consistency of a fuzzy ABox w.r.t. an RBox.

\section{Transitivity in Fuzzy Description Logics}

In classical DLs, a role $R$ is transitive iff for all $a, b, c \in \Delta^{\mathcal{I}},\langle a, b\rangle \in R^{\mathcal{I}}$ and $\langle b, c\rangle \in R^{\mathcal{I}}$ imply $\langle a, c\rangle \in R^{\mathcal{I}}$. Sattler (1996) shows that, for $a, b, c_{1}, \ldots, c_{n} \in \Delta^{\mathcal{I}}$, if $R$ is transitive, $b$ is an $R$-successor of $a, c_{1}, \ldots, c_{n}$ are the $R$-successors of $b$, and $a \in(\forall R . C)^{\mathcal{I}}$, then all $R$ successors of $a$ should be instances of $(\forall R . C)^{\mathcal{I}}$, e.g., $b \in(\forall R . C)^{\mathcal{I}}$ because: (i) $\left\langle a, c_{i}\right\rangle \in R^{\mathcal{I}}$ (as $R$ is transitive), (ii) $c_{i} \in C^{\mathcal{I}}$ (as $a \in(\forall R . C)^{\mathcal{I}}$ ) and (iii) $b \in(\forall R . C)^{\mathcal{I}}$ (due to the semantics of $\forall R . C$ ). In other words, this means that the following concept subsumption holds, $\forall R . C \sqsubseteq \forall R .(\forall R . C)$.

The above property suggests that value restrictions on transitive relations $(\forall R . C)$ are propagated along the path of individuals. This propagation is crucial for reasoning algorithms in order to retain the tree-model property (Baader et al., 2002a), which is a property that leads to decidable decision procedures (Vardi, 1997). Our goal in the rest of the section is to investigate this property in the context of fuzzy Description Logics that allow for transitive role axioms. We have to determine if similar propagation occurs and if it is, to find out the membership degree that the propagation carries to subsequent objects. This is the first time that such an investigation is presented in the literature.

In fuzzy DLs, objects are instances of all possible fuzzy concepts in some degree, ranging over the interval $[0,1]$. As we have shown in Section 3, a fuzzy role $R$ is transitive iff, for all $a, c \in \Delta^{\mathcal{I}}, R^{\mathcal{I}}(a, c) \geq \sup _{b \in \Delta^{\mathcal{I}}} \min \left(R^{\mathcal{I}}(a, b), R^{\mathcal{I}}(b, c)\right)$. Since this holds for the supremum, for an arbitrary $b \in \Delta^{\mathcal{I}}$ we have that, $R^{\mathcal{I}}(a, c) \geq \min \left(R^{\mathcal{I}}(a, b), R^{\mathcal{I}}(b, c)\right)$ and by applying fuzzy complement in both sides we get, $c\left(R^{\mathcal{I}}(a, c)\right) \leq c\left(\min \left(R^{\mathcal{I}}(a, b), R^{\mathcal{I}}(b, c)\right)\right)$. In what follows, we will show that not only value restrictions, but also existential restrictions on transitive 
roles $(\exists R . C)$ are being propagated, so as to satisfy infimum and supremum restrictions. Now we look at the value restrictions. Let $a, b \in \Delta^{\mathcal{I}}$ be objects in $\Delta^{\mathcal{I}}$ and $R$ a transitive role. If $(\forall R . C)^{\mathcal{I}}(a) \geq v_{a}$, we have (note that $c$ below represents fuzzy complement)

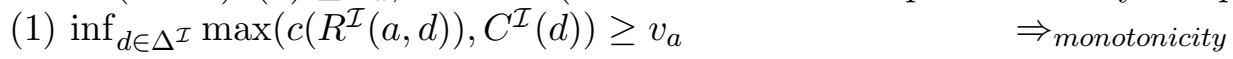

(2) $\inf _{d \in \Delta^{\mathcal{I}} \max }\left(c\left(\min \left(R^{\mathcal{I}}(a, b), R^{\mathcal{I}}(b, d)\right)\right), C^{\mathcal{I}}(d)\right) \geq v_{a} \quad \Rightarrow_{\text {De Morgan }}$

(3) $\inf _{d \in \Delta^{\mathcal{I}}} \max \left(\max \left(c\left(R^{\mathcal{I}}(a, b)\right), c\left(R^{\mathcal{I}}(b, d)\right)\right), C^{\mathcal{I}}(d)\right) \geq v_{a} \quad \Rightarrow_{\text {associativity }}$

(4) $\inf _{d \in \Delta^{\mathcal{I}}} \max \left(c\left(R^{\mathcal{I}}(a, b)\right), \max \left(c\left(R^{\mathcal{I}}(b, d)\right), C^{\mathcal{I}}(d)\right)\right) \geq v_{a} \quad \Rightarrow_{\text {Lemma } 2.2}$

(5) $\max \left(c\left(R^{\mathcal{I}}(a, b)\right), \inf _{d \in \Delta^{\mathcal{I}}} \max \left(c\left(R^{\mathcal{I}}(b, d)\right), C^{\mathcal{I}}(d)\right)\right) \geq v_{a} \Rightarrow$

(6) $\max \left(c\left(R^{\mathcal{I}}(a, b)\right),(\forall R . C)^{\mathcal{I}}(b)\right) \geq v_{a}$,

which means either $c\left(R^{\mathcal{I}}(a, b)\right) \geq v_{a}$ or $(\forall R . C)^{\mathcal{I}}(b) \geq v_{a}$. There are some remarks here. Firstly, the above $b$ is an arbitrary object in $\Delta^{\mathcal{I}}$. In other words, for any object $x \in \Delta^{\mathcal{I}}$, if $c\left(R^{\mathcal{I}}(a, x)\right)<v_{a}$, we have $(\forall R . C)^{\mathcal{I}}(x) \geq v_{a}$. Similarly, if $(\forall R . C)^{\mathcal{I}}(a)>v_{a} \geq c\left(R^{\mathcal{I}}(a, x)\right)$, we have $(\forall R . C)^{\mathcal{I}}(x)>v_{a}$. Hence, the following result is obtained.

Corollary 4.1 If $(\forall R . C)^{\mathcal{I}}(a) \triangleright n$ and $\operatorname{Trans}(R)$ then, in an $f_{K D}-D L,(\forall R .(\forall R . C))^{\mathcal{I}}(a) \triangleright n$ holds.

Now, let $a, b \in \Delta^{\mathcal{I}}, R$ a transitive role and consider the case $(\exists R . C)^{\mathcal{I}}(a) \leq e_{a}$. By applying a fuzzy complement in both sides of the inequation, and since fuzzy complements are monotonic decreasing, we obtain $c\left((\exists R \cdot C)^{\mathcal{I}}(a)\right) \geq c\left(e_{a}\right)$. Based on the semantics of the language this can be rewritten as $(\neg(\exists R . C))^{\mathcal{I}}(a) \geq c\left(e_{a}\right)$ and by using the concept equivalences presented in the previous section we have, $(\forall R .(\neg C))^{\mathcal{I}}(a) \geq c\left(e_{a}\right)$. Hence, by using the above results for value restrictions we can conclude that for any object $x \in \Delta^{\mathcal{I}}$, if $c\left(R^{\mathcal{I}}(a, x)\right)<c\left(e_{a}\right) \Rightarrow R^{\mathcal{I}}(a, x)>e_{a}$, then $(\forall R .(\neg C))^{\mathcal{I}}(x) \geq c\left(e_{a}\right) \Rightarrow(\exists R . C)^{\mathcal{I}}(x) \leq e_{a}$ and similarly, if $(\forall R .(\neg C))^{\mathcal{I}}(a)>c\left(e_{a}\right) \geq c\left(R^{\mathcal{I}}(a, x)\right)$, i.e. $(\exists R . C)^{\mathcal{I}}(a)<c\left(e_{a}\right) \leq R^{\mathcal{I}}(a, x)$, we have $(\forall R .(\neg C))^{\mathcal{I}}(x)>c\left(e_{a}\right)$ and thus $(\exists R . C)^{\mathcal{I}}(x)<e_{a}$. Hence, again we are able to show the next result.

Corollary 4.2 If $(\exists R . C)^{\mathcal{I}}(a) \triangleleft n$ and $\operatorname{Trans}(R)$ then, in an $f_{K D}-D L,(\exists R .(\exists R . C))^{\mathcal{I}}(a) \triangleleft n$ holds.

The above results will be used in properties 11 and 12 in Definition 5.1.

\section{Reasoning with Transitive and Inverse Roles in $\mathbf{f}_{K D^{-}}$-DLs}

In the current section we will show how to reason about transitive and inverse roles in the context of fuzzy DLs, thus providing a reasoning algorithm for the $\mathrm{f}_{K D^{-}} \mathcal{S I}$ language. This algorithm can be used in order to provide efficient implementations for applications that do not need the expressive power of $\mathrm{f}_{K D^{-}} \mathcal{S} \mathcal{H} \mathcal{I} \mathcal{N}$.

In section 3, we have shown that most inference services of fuzzy DLs, like entailment and subsumption, can be reduced to the problem of ABox consistency checking w.r.t. an RBox. As other tableaux algorithms, our tableaux algorithm for checking ABox consistency tries to prove the satisfiability of an assertion by constructing, for an $\mathrm{f}_{K D^{-}} \mathcal{S I}$ ABox $\mathcal{A}$, a fuzzy tableau of $\mathcal{A}$, i.e., an abstraction of a model of $\mathcal{A}$. Given the notion of a fuzzy tableau, it is quite straightforward to prove the algorithm is a decision procedure for ABox 
consistency. The fuzzy tableau we present here can be seen as an extension of the tableau presented by Horrocks et al. (2000) to handle with degrees. The first such extension was presented by Stoilos et al. (2005b), but here we will revise that definition.

Without loss of generality, we assume all concepts $C$ occurring in $\mathcal{A}$ to be in negation normal form (NNF) (Hollunder, Nutt, \& Schmidt-Schaus, 1990); i.e., negations occur in front of concept names only. $\mathrm{A}_{K D^{-}} \mathcal{S I}$-concept can be transformed into an equivalent one in NNF by pushing negations inwards using a combination of the De Morgan laws (which are satisfied by the operations we defined in section 2.3). Next, for a fuzzy concept $D$, we will denote by $\operatorname{sub}(D)$ the set that contains $D$ and it is closed under sub-concepts of $D$ (Horrocks \& Sattler, 1999). The set of all sub-concepts of concepts that appear within an $A B o x$ is denoted by $\operatorname{sub}(\mathcal{A})$.

In the following, we use the symbols $\triangleright$ and $\triangleleft$ as a placeholder for the inequalities $\geq$, > and $\leq,<$ and the symbol $\bowtie$ as a placeholder for all types of inequalities. Furthermore, we use the symbols $\bowtie^{-}, \triangleright^{-}$and $\triangleleft^{-}$to denote their reflections; e.g., the reflection of $\leq$is $\geq$ and that of $>$ is $<$.

Definition 5.1 If $\mathcal{A}$ is an $f_{K D^{-}} \mathcal{S I}$ ABox, $\mathcal{R}$ an $f_{K D^{-}} \mathcal{S I} R B o x, \boldsymbol{R}_{\mathcal{A}}$ is the set of roles occurring in $\mathcal{A}$ and $\mathcal{R}$ together with their inverses and $\mathbf{I}_{\mathcal{A}}$ is the set of individuals in $\mathcal{A}$, a fuzzy tableau $T$ for $\mathcal{A}$ with respect to $\mathcal{R}$, is defined to be a quadruple $(\boldsymbol{S}, \mathcal{L}, \mathcal{E}, \mathcal{V})$ such that: $\boldsymbol{S}$ is a set of elements, $\mathcal{L}: \boldsymbol{S} \times \operatorname{sub}(\mathcal{A}) \rightarrow[0,1]$ maps each element and concept, that is a member of $\operatorname{sub}(\mathcal{A})$, to the membership degree of that element to the concept, $\mathcal{E}: \boldsymbol{R}_{\mathcal{A}} \times \boldsymbol{S} \times \boldsymbol{S} \rightarrow[0,1]$ maps each role of $\boldsymbol{R}_{\mathcal{A}}$ and pair of elements to the membership degree of the pair to the role, and $\mathcal{V}: \mathbf{I}_{\mathcal{A}} \rightarrow \boldsymbol{S}$ maps individuals occurring in $\mathcal{A}$ to elements in $\boldsymbol{S}$. For all $s, t \in \boldsymbol{S}$, $C, D \in \operatorname{sub}(\mathcal{A}), n \in[0,1]$ and $R \in \boldsymbol{R}_{\mathcal{A}}$, T satisfies:

1. $\mathcal{L}(s, \perp)=0$ and $\mathcal{L}(s, \top)=1$ for all $s \in \boldsymbol{S}$,

2. If $\mathcal{L}(s, \neg A) \bowtie n$, then $\mathcal{L}(s, A) \bowtie^{-} 1-n$,

3. If $\mathcal{L}(s, C \sqcap D) \triangleright n$, then $\mathcal{L}(s, C) \triangleright n$ and $\mathcal{L}(s, D) \triangleright n$,

4. If $\mathcal{L}(s, C \sqcup D) \triangleleft n$, then $\mathcal{L}(s, C) \triangleleft n$ and $\mathcal{L}(s, D) \triangleleft n$,

5. If $\mathcal{L}(s, C \sqcup D) \triangleright n$, then $\mathcal{L}(s, C) \triangleright n$ or $\mathcal{L}(s, D) \triangleright n$,

6. If $\mathcal{L}(s, C \sqcap D) \triangleleft n$, then $\mathcal{L}(s, C) \triangleleft n$ or $\mathcal{L}(s, D) \triangleleft n$,

7. If $\mathcal{L}(s, \forall R . C) \triangleright n$, then $\mathcal{E}(R,\langle s, t\rangle) \triangleright^{-} 1-n$ or $\mathcal{L}(t, C) \triangleright n$,

8. If $\mathcal{L}(s, \exists R$.C $\triangleleft n$, then $\mathcal{E}(R,\langle s, t\rangle) \triangleleft n$ or $\mathcal{L}(t, C) \triangleleft n$,

9. If $\mathcal{L}(s, \exists R . C) \triangleright n$, then there exists $t \in \boldsymbol{S}$ such that $\mathcal{E}(R,\langle s, t\rangle) \triangleright n$ and $\mathcal{L}(t, C) \triangleright n$,

10. If $\mathcal{L}(s, \forall R . C) \triangleleft n$, then there exists $t \in \boldsymbol{S}$ such that $\mathcal{E}(R,\langle s, t\rangle) \triangleleft^{-} 1-n$ and $\mathcal{L}(t, C) \triangleleft n$,

11. If $\mathcal{L}(s, \exists R . C) \triangleleft n$ and $\operatorname{Trans}(R)$, then $\mathcal{E}(R,\langle s, t\rangle) \triangleleft n$ or $\mathcal{L}(t, \exists R$.C) $\triangleleft n$,

12. If $\mathcal{L}(s, \forall R . C) \triangleright n$, $\operatorname{Trans}(R)$, then $\mathcal{E}(R,\langle s, t\rangle) \triangleright^{-} 1-n$ or $\mathcal{L}(t, \forall R . C) \triangleright n$,

13. $\mathcal{E}(R,\langle s, t\rangle) \bowtie n$ iff $\mathcal{E}(\operatorname{Inv}(R),\langle t, s\rangle) \bowtie n$, 
14. If $(a: C) \bowtie n \in \mathcal{A}$, then $\mathcal{L}(\mathcal{V}(a), C) \bowtie n$,

15. If $(\langle a, b\rangle: R) \bowtie n \in \mathcal{A}$, then $\mathcal{E}(R,\langle\mathcal{V}(a), \mathcal{V}(b)\rangle) \bowtie n$

There are some remarks regarding Definition 5.1. First, observe that we use the notation $\mathcal{E}(R,\langle s, t\rangle)$ instead of simply $\mathcal{E}(R, s, t)$ in order to distinguish between a role $R$ and an ordered pair of nodes $\langle s, t\rangle$. Moreover, in the above definition we are based on the semantics of fuzzy interpretations, presented in Table 2 , in order to find properties of the fuzzy models according to what relation holds between a membership degree, a specific value and an inequality type. Then, based on these properties we would develop tableaux expansion rules, which try to construct such an abstracted model. For example, for property 3, due to the semantics of $C \sqcap D$, we have that if $(C \sqcap D)^{\mathcal{I}}(s) \geq n$, then $C^{\mathcal{I}}(s)=n_{1}, D^{\mathcal{I}}(s)=n_{2}$, with $\min \left(n_{1}, n_{2}\right)=(C \sqcap D)^{\mathcal{I}}(s) \geq n$. Due to the properties of the min norm we can conclude that both $n_{1} \geq n$ and $n_{2} \geq n$, hold. Furthermore, for property 7 , due to the semantics of $\forall R$. $C$, if $(\forall R . C)^{\mathcal{I}}(s) \geq n$ we have, $\max \left(1-R^{\mathcal{I}}(s, t), C^{\mathcal{I}}(s)\right) \geq n$, hence either $1-R^{\mathcal{I}}(s, t) \geq n \Rightarrow R^{\mathcal{I}}(s, t) \leq 1-n$ or $C^{\mathcal{I}}(t) \geq n$. Similarly, we have constructed properties for all possible relations between a node, an $\mathrm{f}_{K D^{-}} \mathcal{S} \mathcal{I}$-concept and a value of the unit interval. Properties 9 and 10 are based on the fact that we assume the existence of only witnessed models. Otherwise, no such assumption could be made. Hence, intuitively a fuzzy tableau is an abstraction of the witnessed models of a fuzzy ABox. Finally, property 14 means that if a fuzzy assertion of the form $(a: C)>n$ exists in a fuzzy ABox, then the membership degree of the node $\mathcal{V}(a)$ to the concept $C$ in the fuzzy tableau, should be strictly greater than $n$. Similarly, with the rest of inequalities as well as with property 15.

We now have to prove the lemma connecting ABox consistency and the existence of a fuzzy tableau for $\mathcal{A}$.

Lemma 5.2 An $f_{K D^{-}} \mathcal{S I} A B o x \mathcal{A}$ is consistent w.r.t. $\mathcal{R}$, iff there exists a fuzzy tableau for $\mathcal{A}$ w.r.t. $\mathcal{R}$.

Proof: For the if direction if $T=(\mathbf{S}, \mathcal{L}, \mathcal{E}, \mathcal{V})$ is a fuzzy tableau for an ABox $\mathcal{A}$ w.r.t. $\mathcal{R}$, we can construct a fuzzy interpretation $\mathcal{I}=\left(\Delta^{\mathcal{I}},{ }^{\mathcal{I}}\right)$ that is a model of $\mathcal{A}$.

An interpretation can be defined as follows:

$$
\begin{aligned}
\Delta^{\mathcal{I}} & =\mathbf{S} \\
a^{\mathcal{I}} & =\mathcal{V}(a), a \in \mathbf{I}_{\mathcal{A}} \\
\top^{\mathcal{I}}(s) & =\mathcal{L}(s, \top) \text { for all } s \in \mathbf{S} \\
\perp^{\mathcal{I}}(s) & =\mathcal{L}(s, \perp) \text { for all } s \in \mathbf{S} \\
A^{\mathcal{I}}(s) & =\mathcal{L}(s, A) \text { for all } s \in \mathbf{S} \text { and concept names } A \\
R^{\mathcal{I}}(s, t) & =\left\{\begin{array}{l}
R_{\mathcal{E}}^{+}(s, t) \text { for all }\langle s, t\rangle \in \mathbf{S} \times \mathbf{S} \text { if Trans }(R) \\
R_{\mathcal{E}}(s, t) \text { for all }\langle s, t\rangle \in \mathbf{S} \times \mathbf{S} \text { otherwise }
\end{array}\right.
\end{aligned}
$$

where $R_{\mathcal{E}}(s, t)$ is a binary fuzzy relation defined as $R_{\mathcal{E}}(s, t)=\mathcal{E}(R,\langle s, t\rangle)$ for all $\langle s, t\rangle \in \mathbf{S} \times \mathbf{S}$, and $R_{\mathcal{E}}^{+}$represents its sup-min transitive closure (Klir \& Yuan, 1995).

To prove that $\mathcal{I}$ is a model of $\mathcal{A}$, we show by induction on the structure of concepts that $\mathcal{L}(s, C) \bowtie n$ implies $C^{\mathcal{I}}(s) \bowtie n$ for any $s \in \mathbf{S}$. First, Property 1 ensures that the top 
and bottom concepts are interpreted correctly. Together with properties 14, 15, and the interpretation of individuals and roles, this implies that $\mathcal{I}$ satisfies each assertion in $\mathcal{A}$. Without loss of generality, in the following, we will only show the cases with $\mathcal{L}(s, C) \geq n$. The rest of the inequalities can be shown in a similar way.

1. If $A$ is a concept name then by definition $n \bowtie \mathcal{L}(s, A)=A^{\mathcal{I}}(s)$.

2. If $\mathcal{L}(s, \neg A) \geq n$, then due to property $2 \mathcal{L}(s, A) \leq 1-n$. By definition of $\mathcal{I}, A^{\mathcal{I}}(s) \leq$ $1-n$, hence $(\neg A)^{\mathcal{I}}(s) \geq c(1-n)=n$.

3. If $\mathcal{L}(s, C \sqcap D) \geq n$, then $\mathcal{L}(s, C) \geq n$ and $\mathcal{L}(s, D) \geq n$. By induction, $C^{\mathcal{I}}(s) \geq n$, $D^{\mathcal{I}}(s) \geq n$, hence $(C \sqcap D)^{\mathcal{I}}(s)=\min \left(C^{\mathcal{I}}(s), D^{\mathcal{I}}(s)\right) \geq n$.

4. If $\mathcal{L}(s, C \sqcup D) \geq n$, then $\mathcal{L}(s, C) \geq n$ or $\mathcal{L}(s, D) \geq n$. By induction either $C^{\mathcal{I}}(s) \geq n$ or $D^{\mathcal{I}}(s) \geq n$ and $(C \sqcup D)^{\mathcal{I}}(s)=\max \left(C^{\mathcal{I}}(s), D^{\mathcal{I}}(s)\right) \geq n$.

5. If $\mathcal{L}(s, \exists R . C) \geq n$, then there exists $t \in \mathbf{S}$ such that, $\mathcal{E}(R,\langle s, t\rangle) \geq n$ and $\mathcal{L}(t, C) \geq$ $n$. By definition $R^{\mathcal{I}}(s, t) \geq n$ and by induction $C^{\mathcal{I}}(t) \geq n$. Hence, $(\exists R . C)^{\mathcal{I}}(s)=$ $\sup _{t \in \Delta^{\mathcal{I}}} \min \left(R^{\mathcal{I}}(s, t), C^{\mathcal{I}}(t)\right) \geq n$.

6. If $\mathcal{L}(s, \forall R . C) \geq n$ and $R^{\mathcal{I}}(s, t)=p$, then either

(a) $\mathcal{E}(R,\langle s, t\rangle)=p$, or

(b) there exist several paths $l \geq 1$ of the form, $\mathcal{E}\left(R,\left\langle s, s_{l_{1}}\right\rangle\right)=p_{l_{1}}, \mathcal{E}\left(R,\left\langle s_{l_{1}}, s_{l_{2}}\right\rangle\right)=$ $p_{l_{2}}, \ldots, \mathcal{E}\left(R,\left\langle s_{l_{m}}, t\right\rangle\right)=p_{l_{m+1}}$. The membership degree $p$ of the pair $\langle s, t\rangle$ to the transitive closure of $R$, would be equal to the maximum degree (since we cannot have infinite number of different paths) of all the minimum degrees for each path. If that degree is such that it is not lower or equal to $1-n\left(\right.$ since $\left.\geq^{-}=\leq\right)$then there exists a path, $k$, where for all degrees:

$$
\mathcal{E}\left(R,\left\langle s_{k_{i}}, s_{k_{i+1}}\right\rangle\right)=p_{k_{i}}, 0 \leq i \leq m, s_{k_{0}} \equiv s, s_{k_{m+1}} \equiv t,
$$

it holds that $p_{k_{i}}>1-n$, because all $p_{k_{i}}$ would be greater or equal than the minimum degree of the path. Hence, due to Property 12 for all $s_{k_{i}}$ we have $\mathcal{L}\left(s_{k_{i}}, \forall R . C\right) \geq n$.

In case $p \leq 1-n$ we have that $\max \left(1-p, C^{\mathcal{I}}(t)\right) \geq n$. In case $p \not \leq 1-n$, then $\mathcal{L}(t, C) \geq n$, so by induction $C^{\mathcal{I}}(t) \geq n$ and thus also $\max \left(1-R^{\mathcal{I}}(s, t), C^{\mathcal{I}}(t)\right) \geq n$. In both cases we have that $(\forall R . C)^{\mathcal{I}}(s) \geq n$.

For the converse, if $\mathcal{I}=\left(\Delta^{\mathcal{I}}, \cdot^{\mathcal{I}}\right)$ is a (witnessed) model of $\mathcal{A}$ w.r.t. $\mathcal{R}$, then a fuzzy tableau $T=(\mathbf{S}, \mathcal{L}, \mathcal{E}, \mathcal{V})$ for $\mathcal{A}$ w.r.t. $\mathrm{R}$ can be defined as:

$$
\begin{aligned}
\mathbf{S} & =\Delta^{\mathcal{I}} \\
\mathcal{E}(R,\langle s, t\rangle) & =R^{\mathcal{I}}(s, t) \\
\mathcal{L}(s, C) & =C^{\mathcal{I}}(s) \\
\mathcal{V}(a) & =a^{\mathcal{I}}
\end{aligned}
$$


1. Property 1 is satisfied since $\mathcal{I}$ is a fuzzy interpretation.

2. Let $\mathcal{L}(s, \neg C) \triangleright n$. The definition of $T$ implies that $(\neg C)^{\mathcal{I}}(s)=n^{\prime} \triangleright n \Rightarrow C^{\mathcal{I}}(s)=$ $1-n^{\prime} \triangleright^{-} 1-n$, so $\mathcal{L}(s, C) \triangleright^{-} 1-n$ and Property 2 is satisfied. Similarly with the inequalities $\triangleleft \in\{\leq,<\}$.

3. Let $\mathcal{L}(s, C \sqcap D) \triangleright n$. The definition of $T$ implies that $(C \sqcap D)^{\mathcal{I}}(s)=n^{\prime} \triangleright n \Rightarrow$ $\min \left(C^{\mathcal{I}}(s), D^{\mathcal{I}}(s)\right)=n^{\prime} \triangleright n$. By definition, $\mathcal{L}(s, C) \triangleright n$ and $\mathcal{L}(s, D) \triangleright n$ and $T$ satisfies Property 3. Property 4 is proved in a similar way.

4. Let $\mathcal{L}(s, C \sqcup D) \triangleright n$. The definition of $T$ implies that $(C \sqcup D)^{\mathcal{I}}(s)=n^{\prime} \triangleright n \Rightarrow$ $\max \left(C^{\mathcal{I}}(s), D^{\mathcal{I}}(s)\right)=n^{\prime} \triangleright n$. By definition of $T$, either $\mathcal{L}(s, C) \triangleright n$ or $\mathcal{L}(s, D) \triangleright n$, and $T$ satisfies Property 5 . Property 6 is proved in a similar way.

5. Let $\mathcal{L}(s, \forall R . C) \triangleright n$. The definition of $T$ implies that $(\forall R . C)^{\mathcal{I}}(s)=n^{\prime} \triangleright n \Rightarrow$ $\inf _{y \in \Delta^{\mathcal{I}}} \max \left(1-R^{\mathcal{I}}(s, y), C^{\mathcal{I}}(y)\right)=n^{\prime} \triangleright n$. This means that for any $t \in \Delta^{\mathcal{I}}$ either $1-R^{\mathcal{I}}(s, t)=n^{\prime} \triangleright n$ or $C^{\mathcal{I}}(t)=n^{\prime} \triangleright n$, and by definition either $\mathcal{E}(R,\langle s, t\rangle) \triangleright^{-} 1-n$ or $\mathcal{L}(t, C) \geq n$. Thus, $T$ satisfies Property 7 . Property 8 is proved in a similar way.

6. Let $\mathcal{L}(s, \exists R . C) \triangleright n$. The definition of $T$ implies that $(\exists R . C)^{\mathcal{I}}(s)=n^{\prime} \triangleright n \Rightarrow$ $\sup _{y \in \Delta^{\mathcal{I}} \min }\left(R^{\mathcal{I}}(s, y), C^{\mathcal{I}}(y)\right)=n^{\prime} \triangleright n$. This means that there exists some $t \in \Delta^{\mathcal{I}}$ with $R^{\mathcal{I}}(s, t)=n^{\prime} \triangleright n$ and $C^{\mathcal{I}}(t)=n^{\prime} \triangleright n$. By definition $t \in \mathbf{S}$ and $T$ satisfies Property 9. Property 10 is proved in a similar way.

7. Property 12 of definition 5.1 is satisfied as a result of the semantics of transitive roles and value restrictions that have been investigated in section 4. Hence, if $(\forall R . C)^{\mathcal{I}}(s) \geq$ $n$, Trans $(R)$ then either $R^{\mathcal{I}}(s, t) \leq 1-n$, or $(\forall R \cdot C)^{\mathcal{I}}(t) \geq n$ holds, otherwise if $(\forall R . C)^{\mathcal{I}}(s)>n$, Trans $(R)$ then either $R^{\mathcal{I}}(s, t)<1-n$ or $(\forall R . C)^{\mathcal{I}}(t)>n$ holds. By definition of $T$ if $\mathcal{L}(s, \forall R . C) \triangleright n$, Trans $(R)$ then either $\mathcal{E}(R,\langle s, t\rangle) \triangleright^{-} 1-n$ or $\mathcal{L}(t, \forall R . C) \triangleright n$. For similar reasons Property 11, holds.

8. $T$ satisfies Property 13 in Definition 5.1 as a direct consequence of the semantics of inverse relations.

9. $T$ satisfies Properties 14 and 15 in Definition 5.1 because $\mathcal{I}$ is a model of $\mathcal{A}$.

\subsection{An Algorithm for Constructing an $\mathbf{f}_{K D^{-}} \mathcal{S} \mathcal{I}$ Fuzzy Tableau}

Now we present a tableaux algorithm that tries to construct, given an $\mathrm{f}_{K D^{-}} \mathcal{S} \mathcal{I}$ ABox $\mathcal{A}$ and an $\mathrm{f}_{K D^{-}} \mathcal{S} \mathcal{I}$ RBox $\mathcal{R}$, a fuzzy tableau for $\mathcal{A}$ w.r.t. $\mathcal{R}$. We prove that this algorithm construct a fuzzy tableau for $\mathcal{A}$ and $\mathcal{R}$ iff there exists a fuzzy tableau for $\mathcal{A}$ and $\mathcal{R}$, and thus decides consistency of $\mathrm{f}_{K D^{-}} \mathcal{S} \mathcal{I}$ ABoxes w.r.t. RBoxes.

Like the tableaux algorithm presented by Horrocks et al. (2000), our algorithm works on completion-forests rather than on completion-trees, since an ABox might contain several individuals with arbitrary roles connecting them. Due to the presence of transitive roles, the termination of the algorithm is ensured by the use of blocking, where an expansion is 
terminated when two individuals on the same path are asserted to belong to the same concepts. As $\mathrm{f}_{K D^{-}} \mathcal{S} \mathcal{I}$ provides both inverse roles and transitive role axioms, our algorithm uses dynamic blocking (Horrocks \& Sattler, 1999); i.e., blocked nodes (and their sub-branches) can be un-blocked and blocked again later. As it was noted by Horrocks and Sattler (1999) this un-blocking and re-blocking technique is crucial in the presence of inverse roles since information might be propagated up the completion-forest and affect other branches. For example consider the nodes $x, y$ and $z$, the edges $\langle x, y\rangle$ and $\langle x, z\rangle$ and suppose that $x$ blocks $y$. In the presence of inverse roles it is possible that $z$ adds information to node $x$, although $z$ is a successor of $x$. In that case the block on $y$ must be broken. Finally, even in cases where a node is blocked and un-blocking does not occur it is necessary to allow some expansion to be performed. For example node $y$ might contain inverse information that if allowed to be propagated upwards can render the completion-forest unsatisfiable. Thus, dynamic blocking uses the notions of directly and indirectly blocked nodes.

Definition 5.3 (Completion-Forest) A completion-forest $\mathcal{F}_{\mathcal{A}}$ for an $f_{K D^{-}} \mathcal{S} \mathcal{I}$ ABox $\mathcal{A}$ is a collection of trees whose distinguished roots are arbitrarily connected by edges. Each node $x$ is labelled with a set $\mathcal{L}(x)=\{\langle C, \bowtie, n\rangle\}$, where $C \in \operatorname{sub}(\mathcal{A}), \bowtie \in\{\geq,>, \leq,<\}$ and $n \in[0,1]$. Each edge $\langle x, y\rangle$ is labelled with a set $\mathcal{L}(\langle x, y\rangle)=\{\langle R, \bowtie, n\rangle\}$, where $R \in \boldsymbol{R}_{\mathcal{A}}$ are (possibly inverse) roles occurring in $\mathcal{A}$. Intuitively, each triple $\langle C, \bowtie, n\rangle(\langle R, \bowtie, n\rangle)$, called membership triple, represents the membership degree and the type of assertion of each node (pair of nodes) to a concept $C \in \operatorname{sub}(\mathcal{A})$ (role $R \in \boldsymbol{R}_{\mathcal{A}}$ ).

If nodes $x$ and $y$ are connected by an edge $\langle x, y\rangle$ with $\langle R, \bowtie, n\rangle \in \mathcal{L}(\langle x, y\rangle)$, then $y$ is called an $R_{\bowtie, n}$-successor of $x$ and $x$ is called an $R_{\bowtie, n}$-predecessor of $y$. If $y$ is an $R_{\bowtie, n}$ successor or an $\operatorname{lnv}(R)_{\bowtie, n}$-predecessor of $x$, then $y$ is called an $R_{\bowtie, n}$-neighbour of $x$. Let $y$ be an $R_{>, n}$-neighbour of $x$, the edge $\langle x, y\rangle$ conjugates with triples $\langle R, \triangleleft, m\rangle$ if $n \geq m$. Similarly, we can extend it to the cases of $R_{\geq, n^{-}}, R_{<, n^{-}}$and $R_{\leq, n^{-}}$neighbours.

$A$ node $x$ is an $R$-successor (resp. $R$-predecessor or $R$-neighbour) of $y$ if it is an $R_{\bowtie, n^{-}}$ successor (resp. $R_{\bowtie, n}$-predecessor or $R_{\bowtie, n}$-neighbour) of $y$ for some role $R$. A node $x$ is a positive (resp. negative) successor (resp. predecessor or neighbour) of $y$ if $\bowtie \in\{>, \geq\}$ (resp. $\bowtie \in\{<, \leq\}$ ). As usual, ancestor is the transitive closure of predecessor.

A node $x$ is blocked iff it is not a root node and it is either directly or indirectly blocked. A node $x$ is directly blocked iff none of its ancestors are blocked, and it has an ancestor $y$ such that $\mathcal{L}(x)=\mathcal{L}(y)$. In this case, we say $y$ directly blocks $x$. A node $x$ is indirectly blocked iff one of its predecessor is blocked.

A node $x$ is said to contain a clash iff there exist two conjugated triples, or one of the following triples within $\mathcal{L}(x)$ :

$$
\begin{aligned}
& \langle\perp, \geq, n\rangle,\langle\top, \leq, n\rangle, \text { for } n>0, n<1 \text { respectively } \\
& \langle\perp,>, n\rangle,\langle\top,<, n\rangle \\
& \langle C,<, 0\rangle,\langle C,>, 1\rangle
\end{aligned}
$$

Moreover, for an edge $\langle x, y\rangle, \mathcal{L}(\langle x, y\rangle)$ is said to contain a clash iff there exist two conjugated triples in $\mathcal{L}(\langle x, y\rangle)$, or if $\mathcal{L}(\langle x, y\rangle) \cup\{\langle\operatorname{Inv}(R), \bowtie, n\rangle \mid\langle R, \bowtie, n\rangle \in \mathcal{L}(\langle y, x\rangle)\}$, and $x, y$ are root nodes, contains two conjugated triples.

The definition of a completion-forest is quite intuitive. Since a fuzzy ABox contains fuzzy assertions of the form $(a: C) \bowtie n$ and $(\langle a, b\rangle: R) \bowtie n$, then the nodes and edges of the forest 


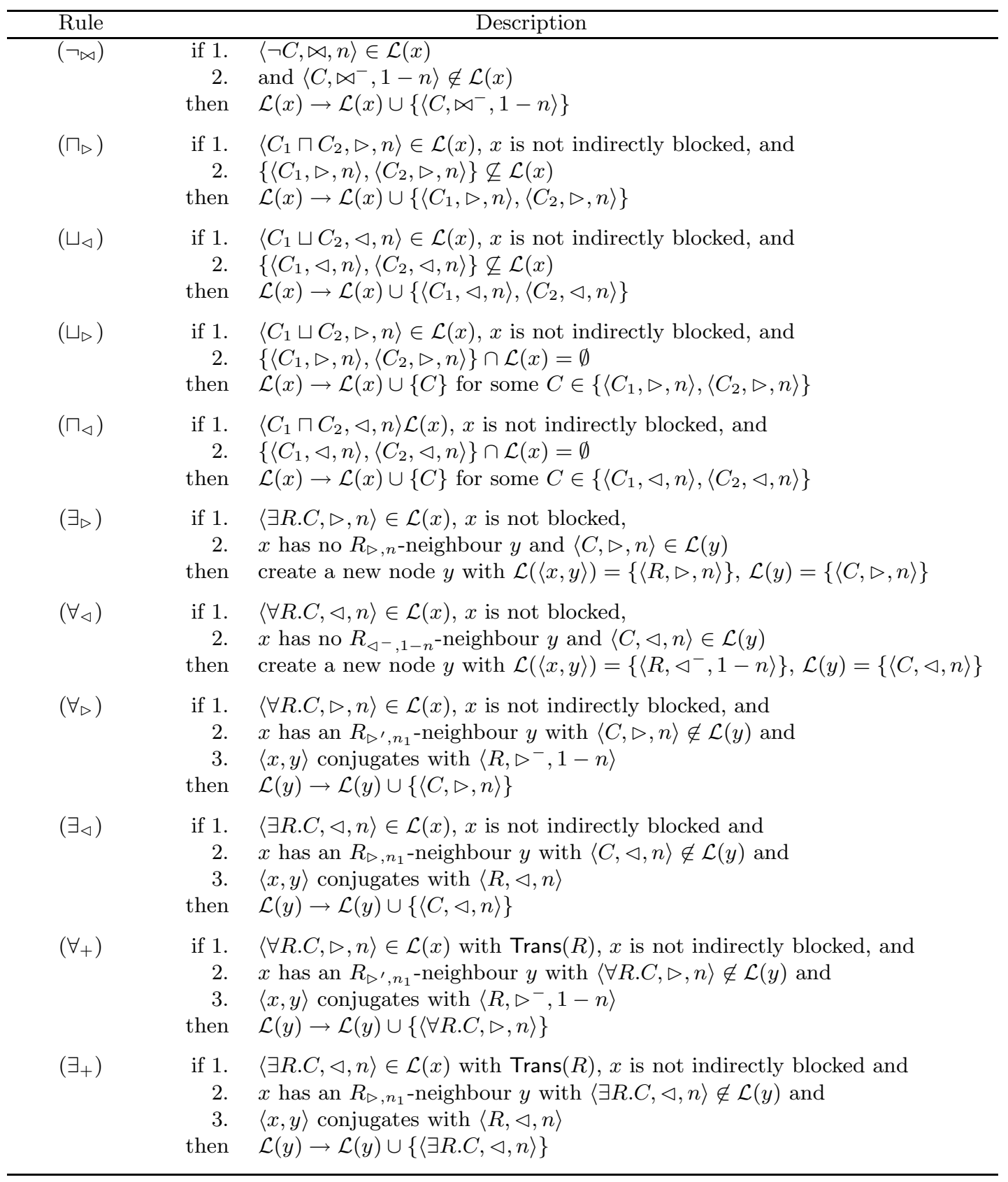

Table 4: $\mathrm{f}_{K D^{-}} \mathcal{S I}$ completion rules

must contain the information about the concept, the type of inequality and the membership degree for every individual, which in the forest is represented by a node.

Definition 5.4 (Tableaux Algorithm) For an $f_{K D^{-}} \mathcal{S I}$ ABox $\mathcal{A}$, the algorithm initialises a forest $\mathcal{F}_{\mathcal{A}}$ to contain (i) a root node $x_{a_{i}}$, for each individual $a_{i} \in \mathbf{I}_{\mathcal{A}}$ occurring in $\mathcal{A}$, labelled with $\mathcal{L}(x)$ such that $\left\{\left\langle C_{i}, \bowtie, n\right\rangle\right\} \subseteq \mathcal{L}\left(x_{a_{i}}\right)$ for each assertion of the form $\left(a_{i}: C_{i}\right) \bowtie n$ 
in $\mathcal{A}$, and (ii) an edge $\left\langle x_{a_{i}}, x_{a_{j}}\right\rangle$, for each assertion $\left(\left\langle a_{i}, a_{j}\right\rangle: R_{i}\right) \bowtie n$ in $\mathcal{A}$, labelled with $\mathcal{L}\left(\left\langle x_{a_{i}}, x_{a_{j}}\right\rangle\right)$ such that $\left\{\left\langle R_{i}, \bowtie, n\right\rangle\right\} \subseteq \mathcal{L}\left(\left\langle x_{a_{i}}, x_{a_{j}}\right\rangle\right)$. Moreover, the algorithm expands $\mathcal{R}$ by adding an axiom $\operatorname{Trans}(\operatorname{Inv}(R))$ for each $\operatorname{Trans}(R) \in \mathcal{R} . \mathcal{F}_{\mathcal{A}}$ is then expanded by repeatedly applying the completion rules from Table 4. The completion forest is complete when, for some node $x, \mathcal{L}(x)$ contains a clash, or none of the completion rules in Table 4 are applicable. The algorithm stops when a clash occurs; it answers ' $\mathcal{A}$ is consistent w.r.t. $\mathcal{R}$ ' iff the completion rules can be applied in such a way that they yield a complete and clash-free conpletion forest, and ' $\mathcal{A}$ is inconsistent w.r.t. $\mathcal{R}$ ' otherwise.

There are some remarks regarding Definition 5.4. The expansion rules are based on the properties of the semantics presented in Definition 5.1. For example, consider the $\left(\forall_{\triangleright}\right)$-rule. Now, if $\langle\forall R . C, \geq, 0.7\rangle \in \mathcal{L}(x)$, and $\langle R, \geq, 0.6\rangle \in \mathcal{L}(\langle x, y\rangle)$, this means that the last triple violates property 7 of Definition 5.1. This property says that the membership degree of the edge $\langle x, y\rangle$ to the role $R$ should be lower or equal than the degree $1-0.7$, otherwise the membership degree of $y$ to $C$ should be greater or equal than 0.7. Interpreted to membership triples this means that if a triple of the form $\langle R, \geq, n\rangle$ exists in $\mathcal{L}(\langle x, y\rangle)$, then $n \leq 1-0.7$, or if the triple is of the form $\langle R,>, n\rangle$, then $n<1-0.7$. In order to discover if these restrictions are violated the $\left(\forall_{\triangleright}\right)$-rule compares the triples of the edge $\langle x, y\rangle$ with the artificial triple $\langle R, \leq, 0.3\rangle$ against conjugation. In the present case conjugation occurs, thus we should add the triple $\langle C, \geq, 0.7\rangle$ to the label of $y$. Similar arguments hold for the rest of the properties. Please note that artificial triples are not added in the completion-forest but are only used to perform checks on membership degrees. Secondly, in the above tableaux algorithm, we see that we are dealing with finite number of membership degrees. In fact, from Table 4, we can see that for an arbitrary fuzzy assertion of the form $(x: D) \bowtie n$ either value $n$ or its complement $c(n)$ appear in the expansion of a node $x$ where $\langle D, \bowtie, n\rangle \in \mathcal{L}(x)$. The finite property of the membership degrees makes blocking possible in our algorithm. This property is a consequence of the fuzzy operations used in our context, i.e. the Gödel tnorm and t-conorm, the Lukasiewicz complement and Kleene-Dienes fuzzy implication and it usually does not hold for other combinations of fuzzy operations. Finding an appropriate blocking condition when other norms are used in combination with Description Logics that include transitive relations is an open research issue. Finally, it is worth noting that since we assume all concepts to be in their negation normal form the $\left(\neg_{\bowtie}\right)$-rule only applies to concept names. But, since we employ a rule for handling negated concepts this is not absolutely necessary in fuzzy DLs. Hence, we are able to not produce the NNF form of negated concepts and apply the $(\neg \bowtie)$-rule directly on them. This might be the base for optimization, since we might be able to identify clashes earlier, or for generalizations to other norm operations, since in that case we might not be able to produce the NNF of negated concepts. In either case, the proof of lemma 5.9 would require a slight modification in order to correctly interpret negated concepts.

Example 5.5 Let us see some examples of applications of expansion rules.

- $(\forall \geq)$ : Let $\langle\forall R . C, \geq, 0.7\rangle \in \mathcal{L}(x)$ and $\langle\operatorname{Inv}(R),>, 0.3\rangle \in \mathcal{L}(\langle y, x\rangle)$. According to the definition of an $R$-neighbour, $y$ is an $R_{>, 0.3}$-neighbour, hence $\langle x, y\rangle$ conjugates with $\langle R, \leq, 0.3\rangle$, and additionally $\langle C, \geq, 0.3\rangle \notin \mathcal{L}(y)$. Thus, we should add $\langle C, \geq, 0.3\rangle$ in $\mathcal{L}(y)$. 
- $(\exists \geq):$ Let $\langle\exists \operatorname{Inv}(R) . C, \geq, 0.7\rangle \in \mathcal{L}(x)$. Then create a new node $y$ in the forest and set, $\langle\operatorname{lnv}(R), \geq, 0.7\rangle \in \mathcal{L}(\langle x, y\rangle),\langle C, \geq, 0.7\rangle \in \mathcal{L}(y)$.

- $\left(\exists_{+}\right):$Let $\langle\exists \operatorname{Inv}(R) . C,<, 0.5\rangle \in \mathcal{L}(x),\langle\operatorname{Inv}(R), \geq, 0.7\rangle \in \mathcal{L}(\langle x, y\rangle)$ and $\operatorname{Trans}(R) . \quad y$ is an $\operatorname{Inv}(R)_{\geq 0.7}$-neighbour of $x$, hence $\langle x, y\rangle$ conjugates with $\langle\operatorname{Inv}(R),<, 0.5\rangle$, and additionally $\langle\exists \operatorname{Inv}(R) . C,<, 0.5\rangle \notin \mathcal{L}(y)$. Hence, $\langle\exists \operatorname{Inv}(R) . C,<, 0.5\rangle$ should be added in $\mathcal{L}(y)$.

Now we can revisit example 3.3 to see how the procedure presented in this section can be used to determine the consistency of the ABox.

Example 5.6 Recall that our fuzzy ABox was $\mathcal{A}=\left\{\left(\left\langle\mathrm{o}_{1}, \mathrm{o}_{2}\right\rangle\right.\right.$ : isPartOf $) \geq 0.8,\left(\left\langle\mathrm{o}_{2}, \mathrm{o}_{3}\right\rangle\right.$ : isPartOf $) \geq 0.9,\left(\mathrm{o}_{2}:\right.$ Body $) \geq 0.85$ and $\left(\mathrm{o}_{1}:\right.$ Arm $\left.) \geq 0.75\right\}$, and that we wanted to test the consistency of the fuzzy $A B o x \mathcal{A}^{\prime}=\mathcal{A} \cup\left\{\left(\mathrm{o}_{3}: \exists \operatorname{lnv}(\right.\right.$ isPartOf $)$. Body $\sqcap \exists \operatorname{lnv}($ isPartOf $)$. Arm $)<$ $0.75\}$, w.r.t. $\mathcal{R}=\{\operatorname{Trans}($ isPartOf) $\}$. According to Definition 5.4 the algorithm initializes a completion-forest to contain the following triples (note that we have a node $x_{\mathrm{O}_{i}}$ for each individual $\mathrm{o}_{i}$ ):

$$
\begin{aligned}
& \text { (1) } \quad \text { isPartOf, } \geq, 0.8\rangle \in \mathcal{L}\left(\left\langle x_{\mathrm{O}_{1}}, x_{\mathrm{O}_{2}}\right\rangle\right) \\
& \text { (2) } \quad \text { isPartOf, } \geq, 0.9\rangle \in \mathcal{L}\left(\left\langle x_{\mathrm{O}_{2}}, x_{\mathrm{O}_{3}}\right\rangle\right) \\
& \text { (3) }\langle\text { Body, } \geq, 0.85\rangle \in \mathcal{L}\left(x_{\mathrm{O}_{2}}\right) \\
& \text { (4) }\langle\text { Arm, } \geq, 0.75\rangle \in \mathcal{L}\left(x_{\mathrm{O}_{1}}\right) \\
& \text { (5) }\left\langle\exists \text { isPartOf }^{-} \text {. Body } \sqcap \text { } \exists \text { isPartOf }^{-} \text {.Arm, }<, 0.75\right\rangle \in \mathcal{L}\left(x_{\mathrm{O}_{3}}\right)
\end{aligned}
$$

Furthermore, the algorithm expands $\mathcal{R}$ by adding the axiom Trans(isPartOf ${ }^{-}$). Please note that for simplicity we have not expanded the concepts Arm and Body in the membership triples. Subsequently, by applying expansion rules from Table 4 we have the following steps:

(6) $\left\langle\exists\right.$ isPartOf $^{-}$. Body, $\left.<, 0.75\right\rangle \in \mathcal{L}\left(x_{\mathrm{O}_{3}}\right) \mid\left\langle\exists\right.$ isPartOf $^{-}$.Arm, $\left.<, 0.75\right\rangle \in \mathcal{L}\left(x_{\mathrm{O}_{3}}\right) \quad\left(\Pi_{<}\right)$

Hence at this point we have two possible completion forests. For the first one we have,

$$
\begin{array}{lll}
\left(6_{1}\right) & \left\langle\exists \text { isPartOf }^{-} . \text {Body, },<, 0.75\right\rangle \in \mathcal{L}\left(x_{\mathrm{O}_{3}}\right) & \\
\left(7_{1}\right) & \langle\text { Body },<, 0.75\rangle \in \mathcal{L}\left(x_{\mathrm{O}_{2}}\right) & \left(\exists_{<}\right):\left(6_{1}\right),(2) \\
\left(8_{1}\right) & \operatorname{clash}\left(7_{1}\right) \text { and }(3) &
\end{array}
$$

while for the second possible completion-forest we have.

$$
\begin{aligned}
& \left.\left(6_{2}\right) \quad\left\langle\exists \text { isPartOf }^{-} \text {.Arm }\right),<, 0.75\right\rangle \in \mathcal{L}\left(x_{\mathrm{O}_{3}}\right) \\
& \left(7_{2}\right) \quad\langle\text { Arm, }<, 0.75\rangle \in \mathcal{L}\left(x_{\mathrm{O}_{2}}\right) \quad\left(\exists_{<}\right):\left(6_{2}\right),(2) \\
& \left.\left(8_{2}\right) \quad\left\langle\exists \text { isPartOf }^{-} \text {.Arm }\right),<, 0.75\right\rangle \in \mathcal{L}\left(x_{\mathrm{O}_{2}}\right) \quad\left(\exists_{+}\right):\left(6_{2}\right),(2) \\
& \left(9_{2}\right) \quad\langle\text { Arm, }<, 0.75\rangle \in \mathcal{L}\left(x_{\mathrm{O}_{1}}\right) \quad\left(\exists_{<}\right):\left(8_{2}\right),(1) \\
& \left(10_{2}\right) \quad \text { clash }\left(9_{2}\right) \text { and }(4)
\end{aligned}
$$

Thus, since all possible expansions result to a clash, $\mathcal{A}^{\prime}$ is inconsistent the knowledge base entails the fuzzy assertion. 
Example 5.7 Consider the fuzzy knowledge base $\Sigma=\langle\mathcal{T}, \mathcal{A}, \mathcal{R}\rangle$, with TBox $\mathcal{T}=\{C \equiv$ $\left.\forall R^{-} .\left(\forall P^{-} . \neg A\right)\right\}, A B o x \mathcal{A}=\{(\mathrm{a}: A) \geq 0.8,(\langle\mathrm{a}, \mathrm{b}\rangle: P) \geq 0.8,(\mathrm{~b}: C) \geq 0.8,(\mathrm{~b}: \exists R . C) \geq$ $0.8,(\mathrm{~b}: \forall R .(\exists R . C)) \geq 0.8\}$ and $R B o x \mathcal{R}=\{\operatorname{Trans}(R)\}$. First the algorithm expands $\mathcal{R}$ by adding axiom $\operatorname{Trans}\left(R^{-}\right)$. Then, in order to check the consistency of $\mathcal{A}$ w.r.t. $\mathcal{T}$ and $\mathcal{R}$ the algorithm initializes the following completion-forest:

$$
\begin{array}{ll}
(1) & \langle A, \geq, 0.8\rangle \in \mathcal{L}\left(x_{\mathrm{a}}\right) \\
(2) & \langle C, \geq, 0.8\rangle \in \mathcal{L}\left(x_{\mathrm{b}}\right) \\
(3) & \langle\exists R . C, \geq, 0.8\rangle \in \mathcal{L}\left(x_{\mathrm{b}}\right) \\
(4) & \langle\forall R .(\exists R . C), \geq, 0.8\rangle \in \mathcal{L}\left(x_{\mathrm{b}}\right) \\
(5) & \langle P, \geq, 0.8\rangle \in \mathcal{L}\left(x_{\mathrm{a}}, x_{\mathrm{b}}\right) .
\end{array}
$$

Then, we get the following application of expansion rules,

$$
\begin{array}{lll}
(6) & \langle C, \geq, 0.8\rangle \in \mathcal{L}\left(x_{\mathrm{O}_{1}}\right),\langle R, \geq, 0.8\rangle \in \mathcal{L}\left(x_{\mathrm{b}}, x_{\mathrm{O}_{1}}\right) & (\exists \geq):(3) \\
(7) & \langle\exists R . C, \geq, 0.8\rangle \in \mathcal{L}\left(x_{\mathrm{O}_{1}}\right) & \left(\forall_{\geq}\right):(4) \\
(8) & \langle\forall R .(\exists R . C), \geq, 0.8\rangle \in \mathcal{L}\left(x_{\mathrm{O}_{1}}\right) & \left(\forall_{+}\right):(4)
\end{array}
$$

As we can see $\mathcal{L}\left(x_{\mathrm{b}}\right)=\mathcal{L}\left(x_{\mathrm{O}_{1}}\right)$, hence $x_{\mathrm{O}_{1}}$ is blocked by $x_{\mathrm{b}}$. On the other hand it is not indirectly blocked. Hence, since $\left\langle\forall R^{-} .\left(\forall P^{-} . \neg A\right), \geq, 0.8\right\rangle \in \mathcal{L}\left(x_{\mathrm{O}_{1}}\right)$ (due to the definition of $C$ in the TBox) we have the following application of expansion rules,

$$
\begin{array}{cll}
(9) & \left\langle\forall P^{-} . \neg A, \geq, 0.8\right\rangle \in \mathcal{L}\left(x_{\mathrm{b}}\right) & (\forall \geq):(6) \\
(10) & \langle\neg A, \geq, 0.8\rangle \in \mathcal{L}\left(x_{\mathrm{a}}\right) & (\forall \geq):(9) \\
(11) & \langle A, \leq, 0.2\rangle \in \mathcal{L}\left(x_{\mathbf{a}}\right) & (\neg \geq):(10) \\
(12) & \text { clash }(11) \text { and }(1) &
\end{array}
$$

Please note that adding $\left\langle\forall P^{-} . \neg A, \geq, 0.8\right\rangle$ to $\mathcal{L}\left(x_{\mathrm{b}}\right)$ causes the blocking of node $x_{\mathrm{O}_{1}}$ to be broken since it no longer holds that $\mathcal{L}\left(x_{\mathrm{b}}\right)=\mathcal{L}\left(x_{\mathrm{O}_{1}}\right)$. Hence, the notions of indirectly blocked nodes and dynamic blocking are crucial in the presence of inverse roles in order to correctly identify consistent and inconsistent ABoxes. Also note that if the algorithm had chosen to expand $x_{\mathrm{O}_{1}}$ (since this node is no more blocked) rather than $x_{\mathrm{b}}$, then it would have created another node, say $x_{\mathrm{O}_{2}}$, for which $\mathcal{L}\left(x_{\mathrm{O}_{1}}\right)=\mathcal{L}\left(x_{\mathrm{O}_{2}}\right)$. Then again $\langle C, \geq, 0.8\rangle$ would be added to $x_{\mathrm{O}_{1}}$, since $x_{\mathrm{O}_{2}}$ would not be indirectly blocked, the block on $x_{\mathrm{O}_{2}}$ would be broken, but then it would hold that $\mathcal{L}\left(x_{\mathrm{b}}\right)=\mathcal{L}\left(x_{\mathrm{O}_{1}}\right)$. Hence $x_{\mathrm{O}_{1}}$ would be permanently blocked while $x_{\mathrm{O}_{2}}$ indirectly blocked. Then the algorithm would have no other choice but to identify the clash in node $x_{\mathbf{a}}$, as it is showed in steps (9) to (12).

\subsection{Decidability of $\mathbf{f}_{K D^{-}} \mathcal{S} \mathcal{I}$}

The soundness and completeness of the algorithm will be demonstrated by proving that for an $\mathcal{S I}$ ABox $\mathcal{A}$, it always terminates and that it returns consistent iff $\mathcal{A}$ is consistent.

Lemma 5.8 (Termination) For each $f_{K D}-\mathcal{S I} A B o x \mathcal{A}$ and $R B o x \mathcal{R}$, the tableaux algorithm terminates, when started for $\mathcal{A}$ and $\mathcal{R}$.

Proof: Let $m=|\operatorname{sub}(\mathcal{A})|, k=\left|\mathbf{R}_{\mathcal{A}}\right|$ and $l$ be the number of different membership degrees appearing in $\mathcal{A}$. Obviously $m$ and $l$ are linear in the length of $\mathcal{A}$. Termination is a consequence of the following properties of the expansion rules: 
1. The expansion rules never remove nodes from the forest or concepts from node labels.

2. Only the $\left(\exists_{\triangleright}\right)$ - or the $\left(\forall_{\triangleleft}\right)$-rule generate new nodes, and each generation is triggered when $\langle\exists R . C, \triangleright, n\rangle$ or $\langle\forall R . C, \triangleleft, n\rangle$ is in a node label where $\exists R . C$ or $\forall R . C$ is in $\operatorname{sub}(\mathcal{A})$. As no nodes can be removed, these rules will not be applied on the same label repeatedly. Since $s u b(\mathcal{A})$ contains at most $m \exists R . C$ or $m \forall R$.C, the out-degree of the forest is bounded by $2 m l$.

3. Nodes are labelled with triples of the form $\langle C, \bowtie, n\rangle$, so there are at most $2^{8 m l}$ different possible labellings for a pair of nodes. Thus, if a path $p$ is of length at least $2^{8 m l}$ (note that concepts that cause non-termination interact either with a value $n$ or with it's negation, and not with both), then there exist two nodes $x, y$ on $p$ which contain the same label. Since a path on which nodes are blocked cannot become longer, paths are of length at most $2^{8 m l}$.

As the previous lemma suggests the tableaux algorithm runs in exponential space. This is due to a well-known problem inherited from the crisp $\mathcal{S I}$ language (Tobies, 2001). Consider for example the following concepts taken from Tobies (2001),

$$
\begin{gathered}
C \equiv \exists R . D \sqcap \forall R .(\exists R . D) \\
D \equiv\left(A_{1} \sqcup B_{1}\right) \sqcap\left(A_{2} \sqcup B_{2}\right) \sqcap \ldots \sqcap\left(A_{n} \sqcup B_{n}\right)
\end{gathered}
$$

where $R$ is a transitive role. Now consider that we want to check the consistency of the fuzzy ABox $\mathcal{A}=\{(a: C) \geq n\}$. Concept $C$ causes the generation of $R_{\geq, n}$-successors $b_{i}$ for which it also holds that $\left(b_{i}: D\right) \geq n$. Now due to the $\sqcup_{\geq}$-rule, which might choose to add either $\left(b_{i}: A_{i}\right) \geq n$ or $\left(b_{i}: B_{i}\right) \geq n$, there are $2^{n}$ possible ways of expanding $D$. Hence, the algorithm might create a path of exponential depth before blocking applies. Tobies (2001) presents an optimized blocking technique that leads to a PSPACE algorithm for $\mathcal{S I}$. This technique involves a refined blocking strategy as well as the modification of the tableaux expansion rules. Investigating the applicability of this technique to $\mathrm{f}_{K D^{-}} \mathcal{S I}$ is an interesting open problem.

Lemma 5.9 (Soundness) If the expansion rules can be applied to an $f_{K D}-\mathcal{S I}$ ABox $\mathcal{A}$ and an $R B o x \mathcal{R}$ such that they yield a complete and clash-free completion-forest, then $\mathcal{A}$ has a fuzzy tableau w.r.t. $\mathcal{R}$.

Proof: Let $\mathcal{F}_{A}$ be a complete and clash-free completion-forest constructed by the tableaux algorithm for $\mathcal{A}$. The construction of a fuzzy tableau $T=(\mathbf{S}, \mathcal{L}, \mathcal{E}, \mathcal{V})$ is based on the construction of a fuzzy model, presented by Straccia (2001):

For a set of triples of the form $\left\langle A, \geq, n_{i}\right\rangle, i$ a positive integer, that might exist within a set of triples $\mathcal{L}(x)$, the maximum value of $n_{i}$ 's is chosen as a membership degree of $x$ to the fuzzy set $A^{\mathcal{I}}$, i.e. the degree $\mathcal{L}(x, A)$ in our case. If the maximum value participates in a triple of the form $\langle A,>, n\rangle$ a small factor $\epsilon$ is added to the maximum. The existence of such a value is ensured by the clash-freeness of $\mathcal{F}_{\mathcal{A}}$. Please also note that without loss of generality we can force all factors $\epsilon$ to be equal. Furthermore, when no triple of the form $\left\langle C, \triangleright, n_{i}\right\rangle \in \mathcal{L}(x)$ exists, while only triples $\left\langle C, \triangleleft, n_{i}\right\rangle \in \mathcal{L}(x)$ do, the membership degree is 
set to 0 . In cases where a value or existential restriction exists as well as a non conjugated relation, special care to the choice of $\epsilon$ has to be made in order not to choose a high value that causes a conjugation in the interpretation. At last, in order to interpret concepts of the form $\neg A$, where $A$ is a concept name, we first compute the maximum degree of a node to the concept $A$ and then use it to compute $\neg A$. The function that returns the maximum degree is denoted by $g l b$ (Straccia, 2001). Please note that the labellings $\mathcal{L}(s, C)$ refer to nodes of the fuzzy tableau, while those of $\mathcal{L}(x)$ to nodes of the completion-forest. A fuzzy tableau can be defined as follows:

$$
\begin{aligned}
& \mathbf{S}=\left\{x \mid x \text { is a node in } \mathcal{F}_{\mathcal{A}} \text {, and } x \text { is not blocked }\right\}, \\
& \mathcal{L}(x, \perp) \quad=\quad 0 \text {, for all } x \in \mathbf{S}, \\
& \mathcal{L}(x, \top)=1 \text {, for all } x \in \mathbf{S} \text {, } \\
& \mathcal{L}(x, C)=g l b\left[\left\langle C, \bowtie, n_{i}\right\rangle\right], \text { for }\left\langle C, \bowtie, n_{i}\right\rangle \in \mathcal{L}(x) x \text { not blocked, } \\
& \mathcal{L}(x, \neg A)=1-\mathcal{L}(x, A) \text {, for all } x \text { in } \mathcal{F}_{\mathcal{A}} \text { not blocked, with }\langle\neg A, \bowtie, n\rangle \in \mathcal{L}(x), \\
& \mathcal{E}(R,\langle x, y\rangle)=\left\{g l b\left[\left\langle R^{*}, \bowtie, n_{i}\right\rangle\right] \mid 1 . y \text { is an } R_{\bowtie, n_{i}} \text {-neighbour of } x\right. \text { or } \\
& \text { 2. }\left\langle R, \bowtie, n_{i}\right\rangle \in \mathcal{L}(\langle x, z\rangle) \text { and } y \text { blocks } z \text { or } \\
& \text { 3. } \left.\left\langle\operatorname{Inv}(R), \bowtie, n_{i}\right\rangle \in \mathcal{L}(\langle y, z\rangle) \text { and } x \text { blocks } z\right\} \text {, } \\
& \mathcal{V}\left(a_{i}\right)=x_{a_{i}} \text {, where } x_{a_{i}} \text { is a root node, }
\end{aligned}
$$

where $R^{*}$ represents either $R$ or $\operatorname{Inv}(R)$. It can be shown that $T$ is a fuzzy tableau for $\mathcal{A}$ w.r.t. $\mathcal{R}$ :

1. Property 1 of Definition 5.1 is satisfied due to the construction of $T$ and because $\mathcal{F}_{\mathcal{A}}$ is clash-free.

2. Property 2 of Definition 5.1 is satisfied because the $\neg$-rule does not apply and we force all factors $\epsilon$ to be equal. Let $\mathcal{L}(x, \neg A)=n_{1} \geq n$. The definition of $T$ implies that $1-n \geq 1-n_{1}=\mathcal{L}(x, A)$.

3. Properties 3-6 of Definition 5.1 are satisfied because none of $\sqcup_{\bowtie}$ nor $\Pi_{\bowtie}$ apply to any $x \in \mathbf{S}$. For example, let $\mathcal{L}(x, C \sqcap D)=n_{1} \geq n$. The definition of $T$ implies that, either $\left\langle C \sqcap D, \geq, n_{1}\right\rangle \in \mathcal{L}(x)$ or $\left\langle C \sqcap D,>, n^{\prime}\right\rangle \in \mathcal{L}(x)$, with $n_{1}=n^{\prime}+\epsilon$. Completeness of $\mathcal{F}_{\mathcal{A}}$ implies that either $\left\langle C, \geq, n_{1}\right\rangle \in \mathcal{L}(x)$ and $\left\langle D, \geq, n_{1}\right\rangle \in \mathcal{L}(x)$ or $\left\langle C,>, n^{\prime}\right\rangle \in \mathcal{L}(x)$ and $\left\langle D,>, n^{\prime}\right\rangle \in \mathcal{L}(x)$. Hence, $\mathcal{L}(s, C)=g l b\left[\left\langle C, \bowtie, n_{i}\right\rangle\right] \geq \mathcal{L}(s, C \sqcap D) \geq n, \mathcal{L}(s, D)=$ glb $=\left[\left\langle C, \bowtie, n_{i}\right\rangle\right] \geq \mathcal{L}(s, C \sqcap D) \geq n$. The rest of properties follow in a similar way.

4. Property 7 in Definition 5.1 is satisfied. Let $x \in \mathbf{S}$ with $\mathcal{L}(x, \forall R . C)=n_{1} \geq n$ and $\mathcal{E}(R,\langle x, y\rangle) \nsupseteq^{-} 1-n$. The definition of $T$ implies that either $\left\langle\forall R . C, \geq, n_{1}\right\rangle \in \mathcal{L}(x)$ or $\left.\langle\forall R . C\rangle,, n^{\prime}\right\rangle \in \mathcal{L}(x)$ with $n_{1}=n^{\prime}+\epsilon$. Moreover, since the glb function does not create an unnecessary conjugation we have that either:

(a) $y$ is an $R_{\triangleright, r}$-neighbour of $x$

(b) $\langle R, \triangleright, r\rangle \in \mathcal{L}(\langle x, z\rangle), y$ blocks $z$ thus $\mathcal{L}(y)=\mathcal{L}(z)$, or

(c) $\langle\operatorname{lnv}(R), \triangleright, r\rangle \in \mathcal{L}(\langle y, z\rangle), x$ blocks $z$, thus $\mathcal{L}(x)=\mathcal{L}(z)$. 
and $\langle R, \triangleright, r\rangle$ or $\langle\operatorname{lnv}(R), \triangleright, r\rangle$ causes conjugation. Hence, in all 3 cases, the $\forall_{\triangleright}$-rule ensures that either $\left\langle C, \geq, n_{1}\right\rangle \in \mathcal{L}(y)$ or $\left\langle C,>, n^{\prime}\right\rangle \in \mathcal{L}(y)$. Thus, either $\mathcal{L}(y, C) \geq n_{1} \geq$ $n$, or $\mathcal{L}(y, C) \geq n^{\prime}+\epsilon=n_{1} \geq n$. The case with $\mathcal{L}(x, \forall R . C)>n$ and $\mathcal{L}(x, \exists R . C) \triangleleft n$, where the latter regards property 8 , are shown in a similar way.

5. Property 9 in Definition 5.1 is satisfied. Let $x \in \mathbf{S}$ with $\mathcal{L}(x, \exists R . C)=n_{1} \geq n$. The definition of $T$ implies that either $\left\langle\exists R . C, \geq, n_{1}\right\rangle \in \mathcal{L}(x)$ or $\left\langle\exists R . C,>, n^{\prime}\right\rangle \in \mathcal{L}(x)$, with $n_{1}=n^{\prime}+\epsilon$. Then the $\exists_{\triangleright}$-rule ensures that there is either:

(a) a predecessor $y$ such that $\left\langle\operatorname{Inv}(R), \geq, n_{1}\right\rangle \in \mathcal{L}(\langle y, x\rangle)$ and $\left\langle C, \geq, n_{1}\right\rangle \in \mathcal{L}(y)$ or dually with $>$ and $n^{\prime}$. Because $y$ is a predecessor of $x$ it cannot be blocked, so $y \in \mathbf{S}, \mathcal{E}(R,\langle x, y\rangle) \geq n_{1} \geq n$ and $\mathcal{L}(y, C) \geq n_{1}$ or $\mathcal{E}(R,\langle x, y\rangle) \geq n^{\prime}+\epsilon=n_{1} \geq n$ and $\mathcal{L}(y, C) \geq n^{\prime}+\epsilon=n_{1} \geq n$.

(b) a successor $y$ such that $\langle R, \geq, n\rangle \in \mathcal{L}(\langle x, y\rangle),\langle C, \geq, n\rangle \in \mathcal{L}(y)$ or dually with $>$ and $n^{\prime}$. If $y$ is not blocked, then $y \in \mathbf{S}$ and $\mathcal{E}(R,\langle x, y\rangle) \geq n_{1}, \mathcal{L}(y, C) \geq n_{1}$ or $\mathcal{E}(R,\langle x, y\rangle) \geq n^{\prime}+\epsilon, \mathcal{L}(y, C) \geq n^{\prime}+\epsilon=n_{1}$. Otherwise, $y$ is blocked by some $z$. Hence, $z \in \mathbf{S}$ and $\left\langle R, \geq, n_{1}\right\rangle \in \mathcal{L}(\langle x, z\rangle),\left\langle C, \geq, n_{1}\right\rangle \in \mathcal{L}(z)$ or $\left\langle R,>, n^{\prime}\right\rangle \in$ $\mathcal{L}(\langle x, z\rangle),\langle C,>, n\rangle \in \mathcal{L}(z)$. In both cases $\mathcal{L}(z, C) \geq n$ and $\mathcal{E}(R,\langle x, z\rangle) \geq n$.

Similar proof applies for $\mathcal{L}(x, \exists R . C)>n$ and also for Property 10 with $\mathcal{L}(x, \forall R . C) \triangleleft n$.

6. Property 12 in Definition 5.1 is satisfied. Let $x \in \mathbf{S}$ with $\mathcal{L}(x, \forall R . C)=n_{1} \geq n$ and $\mathcal{E}(R,\langle x, y\rangle) \nsupseteq^{-} 1-n$. The definition of $T$ implies that either $\left\langle\forall R . C, \geq, n_{1}\right\rangle \in \mathcal{L}(x)$ or $\left.\langle\forall R . C\rangle,, n^{\prime}\right\rangle \in \mathcal{L}(x)$ with $n_{1}=n^{\prime}+\epsilon$. Moreover, since the $g l b$ function does not create an unnecessary conjugation we have that either:

(a) $y$ is an $R_{\triangleright, r}$-neighbour of $x$

(b) $\langle R, \triangleright, r\rangle \in \mathcal{L}(\langle x, z\rangle), y$ blocks $z$ thus $\mathcal{L}(y)=\mathcal{L}(z)$, or

(c) $\langle\operatorname{lnv}(R), \triangleright, r\rangle \in \mathcal{L}(\langle y, z\rangle), x$ blocks $z$, thus $\mathcal{L}(x)=\mathcal{L}(z)$.

and $\langle R, \triangleright, r\rangle$ or $\langle\operatorname{Inv}(R), \triangleright, r\rangle$ causes conjugation. Hence, in all 3 cases, the $\forall_{+^{-}}$ rule ensures that either $\left\langle\forall R . C, \geq, n_{1}\right\rangle \in \mathcal{L}(y)$ or $\left\langle\forall R . C,>, n^{\prime}\right\rangle \in \mathcal{L}(y)$. Thus, either $\mathcal{L}(y, \forall R . C) \geq n_{1} \geq n$, or $\mathcal{L}(y, \forall R . C) \geq n^{\prime}+\epsilon=n_{1} \geq n$. The case with $\mathcal{L}(x, \forall R . C)>n$ and $\mathcal{L}(\exists R . C, \triangleleft, n\rangle$, where the latter regards property 11, are shown in a similar way.

7. Property 13 in Definition 5.1 is satisfied because, if $\mathcal{E}(R,\langle x, y\rangle) \bowtie n$, then either:

(a) $y$ is an $R_{\bowtie, n_{1}}$-neighbour of $x$, so $x$ is an $\operatorname{lnv}(R)_{\bowtie, n_{1}}$-neighbour of $y$.

(b) $\left\langle R, \bowtie, n_{1}\right\rangle \in \mathcal{L}(\langle x, z\rangle)$, and $y$ blocks $z$, so $\left\langle\operatorname{Inv}(\operatorname{Inv}(R)), \bowtie, n_{1}\right\rangle \in \mathcal{L}(\langle x, z\rangle)$

(c) $\left\langle\operatorname{Inv}(R), \bowtie, n_{1}\right\rangle \in \mathcal{L}(\langle y, z\rangle)$ and $x$ blocks $z$.

In all 3 cases, $\mathcal{E}(\operatorname{Inv}(R),\langle y, x\rangle) \bowtie n$.

8. Properties 14 and 15 are satisfied cause of the initialization of the completion-forest and the fact that the algorithm never blocks root nodes. 
Lemma 5.10 (Completeness) Let $\mathcal{A}$ be an $f_{K D}-\mathcal{S I}$ ABox and $\mathcal{R}$ an $R B o x$. If $\mathcal{A}$ has a fuzzy tableau w.r.t. $\mathcal{R}$, then the expansion rules can be applied in such a way that the tableaux algorithm yields a complete and clash-free completion-forest for $\mathcal{A}$ and $\mathcal{R}$.

Proof: Our proof of completeness is based on the proof for crisp DLs presented by Horrocks and Sattler (1999) and Horrocks et al. (2000).

Let $T=(\mathbf{S}, \mathcal{L}, \mathcal{E}, \mathcal{V})$ be a fuzzy tableau for $\mathcal{A}$. Using $T$, we trigger the application of the expansion rules such that they yield a completion-forest $\mathcal{F}_{\mathcal{A}}$ that is both complete and clash-free.

Since we know that $\mathcal{A}$ has a fuzzy tableau $(T)$ we can steer the application of rules such that they yield a complete and clash-free completion-forest. Horrocks and Sattler (1999) and Horrocks et al. (2000) define a mapping $\pi$ which maps nodes of $\mathcal{F}_{\mathcal{A}}$ to elements of $\mathbf{S}$, and guide the application of the non-deterministic rules $\sqcup_{\triangleright}$ and $\Pi_{\triangleleft}$. Our method differs from the one used in crisp DLs (Horrocks \& Sattler, 1999) in the following way. Using the membership degree of a node to a concept, found in the fuzzy tableau, we create artificial triples which are tested against conjugation with the candidate triples that the non-deterministic rules can insert in the completion-forest. The triples that don't cause a conjugation can be added. The modified rules, which are used to guide such an expansion, are presented in Table 5 .

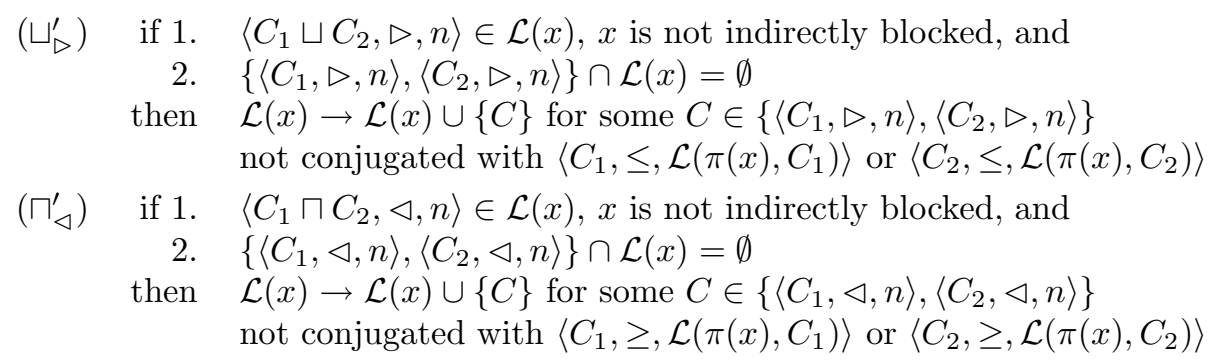

Table 5: The $\sqcup_{\triangleright}^{\prime}$ - and $\sqcap_{\triangleleft}^{\prime}$-rules

$\pi$ ensures that a new fuzzy assertion about the membership degree of a node to a concept, created by a non-deterministic rule, is not more restrictive than the one already known in the fuzzy tableau, thus avoiding possible conjugations. This together with the termination property ensure the completeness of the algorithm.

Theorem 5.11 The tableaux algorithm is a decision procedure for the consistency of $f_{K D^{-}} \mathcal{S I}$ ABoxes and the satisfiability and subsumption of $f_{K D}-\mathcal{S I}$ concepts with respect to simple terminologies.

Theorem 5.11 is an immediate consequence of lemmas 5.1, 5.9 and 5.10. Moreover, as we discussed in section 3, subsumption can be reduced to consistency checking for ABoxes.

\section{Adding Role Hierarchies and Number Restrictions}

In the current section we will provide the necessary extensions of the reasoning algorithm presented in the previous section, in order to provide reasoning support for the fuzzy DL 
language $\mathrm{f}_{K D^{-}} \mathcal{S H \mathcal { I N }}$. To achieve our goal we will extend the results of section 4 by also considering role hierarchies, while we will also provide an investigation on the number restrictions constructor.

In classical DLs, the results of transitive roles and value restrictions obtained by Sattler (1996), were extended by Horrocks and Sattler (1999) to also consider role hierarchies. More precisely, they show that if $x \in(\forall R . C)^{\mathcal{I}},\langle x, y\rangle \in P^{\mathcal{I}}$, $\operatorname{Trans}(P)$ and $P \underline{E} R$, then $y \in(\forall P . C)^{\mathcal{I}}$. In fuzzy DLs that also include role hierarchies we can easily extend the results obtained in section 4. Let $(\forall R . C)^{\mathcal{I}}(x) \geq c_{a}, P^{\mathcal{I}}(x, y)=p$, Trans $(P)$, and $c_{a}, p \in[0,1]$, and consider also that $P \stackrel{E}{\underline{E}}$. Since $P$ is transitive, then $\forall x, y \in \Delta^{\mathcal{I}}$ and for some arbitrary $z \in \Delta^{\mathcal{I}}$ it holds that, $P^{\mathcal{I}}(x, y) \geq \min \left(P^{\mathcal{I}}(x, z), P^{\mathcal{I}}(z, y)\right)$. Due to the semantics of role inclusion axioms we have that $R^{\mathcal{I}}(x, y) \geq \min \left(P^{\mathcal{I}}(x, z), P^{\mathcal{I}}(z, y)\right)$. Then, if we work in a similar way as in section 4 we will get that, $\max \left(c\left(P^{\mathcal{I}}(a, b)\right),(\forall P . C)^{\mathcal{I}}(b)\right) \geq v_{a}$, which means that either $c\left(P^{\mathcal{I}}(a, b)\right) \geq v_{a}$ or $(\forall P . C)^{\mathcal{I}}(b) \geq v_{a}$. A similar result can be obtained for the case where $(\forall R . C)^{\mathcal{I}}(a)>n$. Hence, we get the following result:

Corollary 6.1 If $(\forall R . C)^{\mathcal{I}}(a) \triangleright n$, and $\operatorname{Trans}(P)$ with $P \sqsubseteq R$, then in a $f_{K D}-D L$ it holds that, $(\forall P .(\forall P . C))^{\mathcal{I}}(a) \triangleright n$.

Finally, for the case of negative assertions and existential restrictions the following is easily obtained.

Corollary 6.2 If $(\exists R . C)^{\mathcal{I}}(a) \triangleleft n$, and $\operatorname{Trans}(P)$ with $P \stackrel{\Xi}{ }$, then in a $f_{K D}-D L$ it holds that, $(\exists P .(\exists P . C))^{\mathcal{I}}(a) \triangleleft n$.

Now we will investigate fuzzy number restrictions. Although, from in Table 2 it seems that the semantics of number restrictions are quite complicated, we will see that intuitively, they are quite similar to their crisp counterparts, as long as we also consider membership degrees.

Consider for example the at-least restriction $(\geq p R)^{\mathcal{I}}(a) \geq n$, where $a \in \Delta^{\mathcal{I}}$. Then according to Table 2 we have,

$$
\sup _{b_{1}, \ldots, b_{p} \in \Delta^{\mathcal{I}}} \min _{i=1}^{p}\left\{R^{\mathcal{I}}\left(a, b_{i}\right)\right\} \geq n .
$$

This means that there must be at least $p$ pairs $\left\langle a, b_{i}\right\rangle$, for which $R^{\mathcal{I}}\left(a, b_{i}\right) \geq n$, holds. These semantics are quite intuitive and similar with those of crisp number restrictions. There one would require at least $p$ pairs for which $R^{\mathcal{I}}\left(a, b_{i}\right) \geq 1$, which simply means more than $p$ pairs. Similarly, we can work for $(\geq p R)^{\mathcal{I}}(a)>n$.

Consider now an at-most restriction of the form $(\leq p R)^{\mathcal{I}}(a) \geq n$. Based on the semantics we have the inequation,

$$
\inf _{b_{1}, \ldots, b_{p+1} \in \Delta^{\mathcal{I}}} \max _{i=1}^{p+1}\left\{1-R^{\mathcal{I}}\left(a, b_{i}\right)\right\} \geq n
$$

This means that for all $p+1$ pairs $\left\langle a, b_{i}\right\rangle$, that can be formed, there is at least one pair for which $c\left(R^{\mathcal{I}}\left(a, b_{k}\right)\right) \geq n$, holds. We can also view this equation in a different way which resembles that of crisp number restrictions. From that perspective we can say that, there 
are at most $p$ pairs $\left\langle a, b_{i}\right\rangle$ for which $c\left(R^{\mathcal{I}}\left(a, b_{i}\right)\right)<n$, holds. Similarly, an at-most restriction of the form $(\leq p R)^{\mathcal{I}}(a)>n$ implies that there are at-most $p$ pairs $\left\langle a, b_{i}\right\rangle$, for which it holds that $c\left(R^{\mathcal{I}}\left(a, b_{i}\right)\right) \leq n$. Hence reasoning w.r.t. number restrictions can be reduced to counting how many role assertions $\left(\left\langle a, b_{i}\right\rangle: R\right) \geq n_{i}$ satisfy the above inequalities. If we find that more than $p$ assertions satisfy these inequalities, then we have to non-deterministically merge some of the individual $b_{i}$, as is the case in the crisp $\mathcal{S H \mathcal { I N }}$ algorithm (Horrocks et al., 2000). Now, lets consider the extreme boundaries of 0 and 1 , and apply our equation to the classical at-most restriction, $a \in(\leq p R)^{\mathcal{I}}$. The fuzzy equivalent of this assertions is $(\leq p R)^{\mathcal{I}}(a) \geq 1$, which implies that there are at most $p b_{i} \in \Delta^{\mathcal{I}}$ such that, $c\left(R^{\mathcal{I}}\left(a, b_{i}\right)\right)<1 \Rightarrow R^{\mathcal{I}}\left(a, b_{i}\right)>0$, holds. Since we are only considering 0 and 1 the last inequality implies, $R^{\mathcal{I}}\left(a, b_{i}\right)=1$, i.e. at-most $p$ successors of $a$ in $R^{\mathcal{I}}$.

Dually, we can also provide such intuitive meaning for the cases which involve negative inequalities, like for example the cases of $(\geq p R)^{\mathcal{I}}(a) \leq n_{1}$ or $(\leq p R)^{\mathcal{I}}(a) \leq n_{2}$. Applying negation to the first equation we obtain, $(\neg(\geq p R))^{\mathcal{I}}(a) \geq c\left(n_{1}\right)$, where $c$ is a fuzzy complement. Since the min and max operations satisfy the De Morgan laws, this assertion can be translated to $(\leq(p-1) R)^{\mathcal{I}}(a) \geq 1-n_{1}$, with $p \geq 1$, which is the negation normal form of the former assertion. Similarly, the equation $(\leq p R)^{\mathcal{I}}(a) \leq n_{2}$ can be transformed to the equivalent, $(\geq(p+1) R)^{\mathcal{I}}(a) \geq 1-n_{2}$.

Using the above results we can proceed in the definition of an $\mathrm{f}_{K D^{-}} \mathcal{S H \mathcal { I } N}$ fuzzy tableau. Similarly to 5.1 we consider all concepts to be in NNF. This can be achieved by using the concept equivalences for number restrictions of section 3. The definition of a fuzzy tableau for $\mathrm{f}_{K D^{-}} \mathcal{S} \mathcal{H} \mathcal{I} \mathcal{N}$ first appeared by Stoilos, Stamou, Tzouvaras, Pan, and Horrocks (2005c), but here we have revised that definition to better represent the properties of fuzzy models. Before defining a fuzzy tableau for $\mathrm{f}_{K D^{-}} \mathcal{S H \mathcal { H } N}$ we extend the definition of sub-concepts of a concept $D$ and an $\mathrm{ABox} \mathcal{A}$.

Definition 6.3 For a fuzzy concept $D$ and a role hierarchy $\mathcal{R}$ we define $\operatorname{sub}(D, \mathcal{R})$ to be the smallest set of $f_{K D}-\mathcal{S H \mathcal { I N }}$-concepts that satisfies the following:

- $D \in \operatorname{sub}(D, \mathcal{R})$,

- $\operatorname{sub}(D, \mathcal{R})$ is closed under sub-concepts of $D$, and

- if $\forall S . C \in \operatorname{sub}(D, \mathcal{R})$ and $R \stackrel{*}{=}$, then $\forall R . C \in \operatorname{sub}(D, \mathcal{R})$

- if $\exists S . C \in \operatorname{sub}(D, \mathcal{R})$ and $R \leftleftarrows S$, then $\exists R . C \in \operatorname{sub}(D, \mathcal{R})$

Finally, we define $\operatorname{sub}(\mathcal{A}, \mathcal{R})=\bigcup_{(a: D) \bowtie n \in \mathcal{A}} \operatorname{sub}(D, \mathcal{R})$.

When $\mathcal{R}$ is clear from the context we will simply write $\operatorname{sub}(\mathcal{A})$.

Definition 6.4 If $\mathcal{A}$ is an $f_{K D^{-}} \mathcal{S H \mathcal { I N }} A B o x, \mathcal{R}$ an $f_{K D^{-}} \mathcal{S H \mathcal { I N }} R B$ ox, $\boldsymbol{R}_{\mathcal{A}}$ is the set of roles occurring in $\mathcal{A}$ and $\mathcal{R}$ together with their inverses, $\mathbf{I}_{\mathcal{A}}$ is the set of individuals in $\mathcal{A}$, then a fuzzy tableau $T$ for $\mathcal{A}$ w.r.t. $\mathcal{R}$ is defined as in Definition 5.1 with the additional properties:

11'. If $\mathcal{L}(s, \exists R$.C $) \triangleleft n$, and $\operatorname{Trans}(P)$ with $P \stackrel{\Xi}{=}$, then $\mathcal{E}(P,\langle s, t\rangle) \triangleleft n$ or $\mathcal{L}(t, \exists P . C) \triangleleft n$, 
12'. If $\mathcal{L}(s, \forall R . C) \triangleright n$, and $\operatorname{Trans}(P)$ with $P \stackrel{\Xi}{=}$, then $\mathcal{E}(P,\langle s, t\rangle) \triangleright^{-} 1-n$ or $\mathcal{L}(t, \forall P . C) \triangleright n$,

16. If $\mathcal{E}(R,\langle s, t\rangle) \triangleright n$ and $R \cong S$, then $\mathcal{E}(S,\langle s, t\rangle) \triangleright n$,

17. If $\mathcal{L}(s, \geq p R) \triangleright n$, then $\sharp R^{T}(s, \triangleright, n) \geq p$,

18. If $\mathcal{L}(s, \leq p R) \triangleleft n$, then $\sharp R^{T}\left(s, \triangleleft^{-}, 1-n\right) \geq p+1$,

19. If $\mathcal{L}(s, \geq p R) \triangleleft n$, then $\sharp R_{\neg}^{T}(s, \triangleleft, n) \leq p-1$,

20. If $\mathcal{L}(s, \leq p R) \triangleright n$, then $\sharp R_{\neg}^{T}\left(s, \triangleright^{-}, 1-n\right) \leq p$,

21. If $a \neq b \in \mathcal{A}$, then $\mathcal{V}(a) \neq \mathcal{V}(b)$

where $R^{T}(s, \bowtie, n)=\{t \in S \mid \mathcal{E}(R,\langle s, t\rangle) \bowtie n\}$ returns the set of elements $t \in \boldsymbol{S}$ that participate in $R$ with some element $s$ with a degree, greater or equal, greater, lower or equal or lower than $n$, and $R_{\neg}^{T}(s, \bowtie, n)=\{t \in S \mid \mathcal{E}(R,\langle s, t\rangle) \bowtie n\}$ returns those elements that don't satisfy the given inequality.

As in Definition 5.1, we are based on the semantics of the language and the observations made in the beginning of this section about the properties of the value and existential restrictions, when transitive roles and role hierarchies are involved, and the semantic meaning of at-most and at-least number restrictions. Thus, property 18 should be read as, if $\mathcal{L}(s, \leq p R) \geq n$ then there are at-most $p t \in \mathbf{S}$ such that $\mathcal{E}(R,\langle s, t\rangle) \not \leq 1-n$, i.e. $\mathcal{E}(R,\langle s, t\rangle)>1-n$, and if $\mathcal{L}(s, \leq p R)>n$, then there are at-most $p t \in \mathbf{S}$ such that $\mathcal{E}(R,\langle s, t\rangle) \nless 1-n$.

Lemma 6.5 $A n f_{K D^{-}} \mathcal{S} \mathcal{H} \mathcal{I N} A B o x \mathcal{A}$ is consistent w.r.t. $\mathcal{R}$, iff there exists a fuzzy tableau for $\mathcal{A}$ w.r.t. $\mathcal{R}$.

Proof: The proof of the lemma is similar to that of lemma 5.2 with some important technical details. For the "if" direction, if $T=(\mathbf{S}, \mathcal{L}, \mathcal{E}, \mathcal{V})$ is a fuzzy tableau for $\mathcal{A}$ w.r.t. $\mathcal{R}$, then a model $\mathcal{I}=\left(\Delta,{ }^{\mathcal{I}}\right)$ of $\mathcal{A}$ and $\mathcal{R}$ is constructed as $\Delta^{\mathcal{I}}=\mathbf{S}, a^{\mathcal{I}}=\mathcal{V}(a)$, where $a \in \mathbf{I}_{\mathcal{A}}$, $\top^{\mathcal{I}}(s)=\mathcal{L}(s, \top), \perp^{\mathcal{I}}(s)=\mathcal{L}(s, \perp)$ for all $s \in \mathbf{S}$, and $A^{\mathcal{I}}(s)=\mathcal{L}(s, A)$, for all $s \in \mathbf{S}$ and concept names $A$, while for roles we have:

$$
R^{\mathcal{I}}(s, t)=\left\{\begin{array}{cl}
R_{\mathcal{E}}^{+}(s, t), & \text { if } \operatorname{Trans}(R) \\
\max _{P \underline{\underline{E}} R, P \neq R}\left(R_{\mathcal{E}}(s, t), P^{\mathcal{I}}(s, t)\right) & \text { otherwise }
\end{array}\right.
$$

Observe that the interpretation of non-transitive roles is recursive in order to correctly interpret those non-transitive roles that have a transitive sub-role. From the definition of $R^{\mathcal{I}}$ and property $12^{\prime}$, if $R^{\mathcal{I}}(s, t)=n \in(0,1]$, then either $\mathcal{E}(R,\langle s, t\rangle)=n$, or $\mathcal{E}(R,\langle s, t\rangle)=0$ and there exist several paths $l \geq 1$ of the form,

$$
\mathcal{E}\left(P,\left\langle s, s_{l_{1}}\right\rangle\right)=p_{l_{1}}, \mathcal{E}\left(P,\left\langle s_{l_{1}}, s_{l_{2}}\right\rangle\right)=p_{l_{2}}, \ldots, \mathcal{E}\left(P,\left\langle s_{l_{m}}, t\right\rangle\right)=p_{l_{m+1}}
$$

with $\operatorname{Trans}(P), P \stackrel{\Xi}{=} R$ and $\mathcal{E}(R,\langle s, t\rangle)=\max \left(0, \sup _{l}\left\{\min \left(p_{l_{1}}, \ldots, p_{l_{m+1}}\right)\right\}\right)$.

Property 16 of $\mathcal{I}$ ensures that $\forall s, t \in \Delta^{\mathcal{I}}, P^{\mathcal{I}}(s, t) \leq R^{\mathcal{I}}(s, t)$ for all $P \stackrel{\Xi}{=} R$. Again, by induction on the structure of concepts we can we show that $\mathcal{L}(s, C) \bowtie n$ implies $C^{\mathcal{I}}(s) \bowtie n$ for any $s \in \mathbf{S}$. Here, we restrict our attention on to the cases that are different than lemma 5.2. Similarly to lemma 5.2 we also restrict our attention to the inequalities $\geq$. 
6'. If $\mathcal{L}(s, \forall R . C) \geq n$ and $R^{\mathcal{I}}(s, t)=p$, then either

(a) $\mathcal{E}(R,\langle s, t\rangle)=p$, or

(b) $\mathcal{E}(R,\langle s, t\rangle) \neq p$. Then, there exist several paths $l \geq 1$ of the form, $\mathcal{E}\left(P,\left\langle s, s_{l_{1}}\right\rangle\right)=$ $p_{l_{1}}, \mathcal{E}\left(P,\left\langle s_{l_{1}}, s_{l_{2}}\right\rangle\right)=p_{l_{2}}, \ldots, \mathcal{E}\left(P,\left\langle s_{l_{m}}, t\right\rangle\right)=p_{l_{m+1}}$, with $\operatorname{Trans}(P)$ and $P \underline{\underline{E}} R$. The membership degree $p$ of the pair $\langle s, t\rangle$ to $\left(P^{+}\right)^{\mathcal{I}}$, would be equal to the maximum degree (since we cannot have infinite number of paths) of all the minimum degrees for each path. If that degree is such that it is not lower or equal than $1-n$ then there exists a path $k$ where all degrees

$$
\mathcal{E}\left(P,\left\langle s_{k_{i}}, s_{k_{i+1}}\right\rangle\right)=p_{k_{i}}, 0 \leq i \leq k_{m}, s_{k_{0}} \equiv s, s_{k_{m+1}} \equiv t
$$

are not lower or equal than $1-n$, because all $p_{k_{i}}$ 's would be greater or equal than the minimum degree of the path. Hence, due to property 11, we would have that $\mathcal{L}\left(s_{k_{i}}, \forall P . C\right) \geq n$, for all $1 \leq i \leq k_{m}$.

In case $p \leq 1-n$ we have then $\max \left(1-p, C^{\mathcal{I}}(t)\right) \geq n$. In case $p \not \leq 1-n$ then $\mathcal{L}(t, C) \geq n$, so $C^{\mathcal{I}}(t) \geq n$ and thus also $\max \left(1-p, C^{\mathcal{I}}(t)\right) \geq n$. In both cases $(\forall R . C)^{\mathcal{I}}(s) \geq n$.

7. If $\mathcal{L}(s, \geq p R) \geq n$ then we have, $\mathcal{E}\left(R,\left\langle s, t_{i}\right\rangle\right) \geq n, 1 \leq i \leq p$. By definition $R^{\mathcal{I}}\left(s, t_{i}\right) \geq$ $n$, and thus

$$
n \leq \sup _{t_{i} \in \Delta^{\mathcal{I}}}\left\{\ldots, \min _{i=1}^{p}\left\{R^{\mathcal{I}}\left(s, t_{i}\right)\right\}, \ldots\right\}=(\geq p R)^{\mathcal{I}}(s)
$$

8. If $\langle\leq p R, \geq, n\rangle$ there are at most $p$ pairs $\left\langle s, t_{i}\right\rangle$ for which, $\mathcal{E}\left(R,\left\langle s, t_{i}\right\rangle\right) \not \leq 1-n$, $1 \leq i \leq p$. Thus in all $p+1$-tuples that can be formed there would be at least one pair $\left\langle s, t_{p+1}\right\rangle$ for which $\mathcal{E}\left(R,\left\langle s, t_{p+1}\right\rangle\right) \leq 1-n$ (even if $\left.\mathcal{E}\left(R,\left\langle s, t_{p+1}\right\rangle\right)=0 \leq 1-n\right)$. Hence, $R^{\mathcal{I}}\left(s, t_{p+1}\right) \leq 1-n \Rightarrow c\left(R^{\mathcal{I}}\left(s, t_{p+1}\right)\right) \geq n$. Finally, we have that,

$$
n \leq \inf _{t_{i} \in \Delta^{\mathcal{I}}}\left\{\ldots, \max \left(\max _{i=1}^{p}\left\{c\left(R^{\mathcal{I}}\left(s, t_{i}\right)\right)\right\}, c\left(R^{\mathcal{I}}\left(s, t_{p+1}\right)\right)\right), \ldots\right\}=(\leq p R)^{\mathcal{I}}(s) .
$$

For the converse, if $\mathcal{I}=\left(\Delta^{\mathcal{I}},{ }^{\mathcal{I}}\right)$ is a model for $\mathcal{A}$ w.r.t. $\mathcal{R}$, then a fuzzy tableau $T=$ $(\mathbf{S}, \mathcal{L}, \mathcal{E}, \mathcal{V})$ for $\mathcal{A}$ and $\mathcal{R}$ is defined in exactly the same was an in lemma 5.2. Then,

1. Properties 1-10 and 13 of Definition 5.1 and 16-20 in Definition 6.4 are satisfied as a direct consequence of the semantics of $\mathrm{f}_{K D^{-}} \mathcal{S} \mathcal{H} \mathcal{I} \mathcal{N}$ concepts.

2. Property 12' of Definition 6.4 is satisfied as a consequence of the semantics of transitive roles, role hierarchies and value restrictions that have been investigated in the beginning of the section. Hence, if $(\forall R . C)^{\mathcal{I}}(s) \geq n, P \stackrel{\Xi}{\mathscr{E}} R$ and $\operatorname{Trans}(P)$ then either $P^{\mathcal{I}}(s, t) \leq 1-n$, or $(\forall P . C)^{\mathcal{I}}(t) \geq n$ holds, otherwise if $(\forall R . C)^{\mathcal{I}}(s)>n, P \stackrel{\Xi}{=} R$ and $\operatorname{Trans}(P)$ then either $P^{\mathcal{I}}(s, t)<1-n$ or $(\forall P . C)^{\mathcal{I}}(t)>n$ holds. By definition of $T$ if $\mathcal{L}(s, \forall R . C) \triangleright n, P \stackrel{\Xi}{=} R$ and $\operatorname{Trans}(P)$ then either $\mathcal{E}(P,\langle s, t\rangle) \triangleright^{-} 1-n$ or $\mathcal{L}(t, \forall P . C) \triangleright n$. Similarly, for property 11' of Definition 6.4.

3. $T$ satisfies Properties 14-15 of Definition 5.1 and Property 21 in Definition 6.4 because $\mathcal{I}$ is a model of $\mathcal{A}$. 


\subsection{Constructing an $\mathbf{f}_{K D}-\mathcal{S H} \mathcal{H} \mathcal{N}$ Fuzzy Tableau}

In this section we will show how the algorithm of $\mathrm{f}_{K D^{-}} \mathcal{S I}$, presented in section 5.1 , can

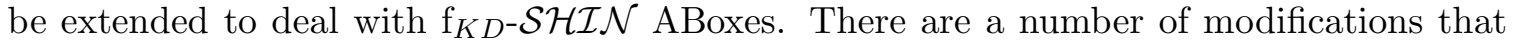
need to be made, like the definition of $R$-neighbours, the $\left(\forall_{+}\right)$- and $\left(\exists_{+}\right)$-rules, the blocking strategy, the clash definition and the addition of rules for number restrictions.

The most important modification from the algorithm of $\mathrm{f}_{K D^{-}} \mathcal{S I}$ is the blocking strategy. As it was noted by Horrocks and Sattler (1999) a DL language that provides inverse roles, transitive role axioms, and number restrictions lacks the finite-model property; i.e. there are

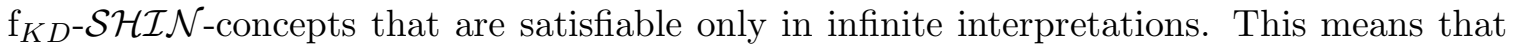
the usual blocking techniques which create a cycle from the predecessor of a blocked node to the blocking one, might fail to construct a correct tableau and due to lemma 6.5 a correct model. It is crucial to remark here the difference between an infinite and a witnessed model, as presented in remark 3.2. Although there are $\mathrm{f}_{K D}-\mathcal{S H \mathcal { I N }}$-concept that are satisfiable in infinite interpretations, these interpretations can still be witnessed w.r.t. the membership degrees. The infinite or finite property of interpretations comes from the constructs of the language, while the witnessed or non-witnessed property comes from the continuity of the fuzzy operators (Hajek, 2005).

Consider for example a node $x$ which contains some triple of the form $\langle\leq 1 R, \geq, 1\rangle$. If a successor of $x$, say $y$, is blocked by some ancestor of $x$, say $z$, the dynamic blocking techniques would create a cycle leading from $x$ back to $z$. But this extra edge $\langle x, z\rangle$ might violate the number restriction on $x$. To overcome this problem the construction of the tableau from the completion-forest is performed by repeatedly copying the sub-tree underneath the node that causes blocking, $z$ in our case. Thus, we are able to obtain an infinite out of the constructed finite forest. Furthermore, in order for copied nodes to be satisfiable in their new locations an extra condition, compared to dynamic blocking has to be employed. The new blocking technique is called pair-wise blocking (Horrocks \& Sattler, 1999); i.e., blocking occurs when two nodes belong to the same set of concepts, their predecessors also belong to the same set of concepts and the edges that connect them are also equal. That way unravelling is guaranteed.

Definition $6.6\left(\mathbf{f}_{K D}-\mathcal{S H I N}\right.$ Completion Forest) First we extend the definition of $R$ successors, predecessors and neighbours. If nodes $x$ and $y$ are connected by an edge $\langle x, y\rangle$ with $\langle P, \triangleright, n\rangle \in \mathcal{L}(\langle x, y\rangle)$, and $P \stackrel{\varpi}{\unrhd}$, then $y$ is called an $R_{\triangleright, n}$-successor of $x$ and $x$ is called an $R_{\triangleright, n}$-predecessor of $y$. If $y$ is an $R_{\triangleright, n}$-successor or an $\operatorname{lnv}(R)_{\triangleright, n}$-predecessor of $x$, then $y$ is called an $R_{\triangleright, n}$-neighbour of $x$.

For a role $R$, a node $x$ in $\mathcal{F}_{\mathcal{A}}$, an inequality $\bowtie$ and a membership degree $n \in[0,1]$ we define: $R_{C}^{\mathcal{F}_{\mathcal{A}}}(x, \bowtie, n)=\left\{y \mid y\right.$ is an $R_{\triangleright^{\prime}, n^{\prime}}$-neighbour of $x$, and $\langle x, y\rangle$ conjugates with $\langle R, \bowtie, n\rangle\}$. Intuitively, this set contains all $R$-neighbours of $x$ that conjugate with a given triple.

A node $x$ is blocked iff it is not a root node and it is either directly or indirectly blocked. A node $x$ is directly blocked iff none of its ancestors is blocked, and it has ancestors $x^{\prime}, y$ and $y^{\prime}$ such that:

1. y is not a root node,

2. $x$ is a successor of $x^{\prime}$ and $y$ a successor of $y^{\prime}$, 
3. $\mathcal{L}(x)=\mathcal{L}(y)$ and $\mathcal{L}\left(x^{\prime}\right)=\mathcal{L}\left(y^{\prime}\right)$ and,

4. $\mathcal{L}\left(\left\langle x^{\prime}, x\right\rangle\right)=\mathcal{L}\left(\left\langle y^{\prime}, y\right\rangle\right)$.

In this case we say that $y$ blocks $x$. A node $y$ is indirectly blocked iff one of its ancestors is blocked, or it is a successor of a node $x$ and $\mathcal{L}(\langle x, y\rangle)=\emptyset$.

For a node $x, \mathcal{L}(x)$ is said to contain a clash if it contains an $f_{K D^{-}} \mathcal{S I}$ clash, or if it contains,

- some triple $\langle\leq p R, \triangleright, n\rangle$ and $x$ has $p+1 R_{\triangleright_{i}, n_{i}}$-neighbours $y_{0}, \ldots, y_{p},\left\langle x, y_{i}\right\rangle$ conjugates with $\left\langle R, \triangleright^{-}, 1-n\right\rangle$ and $y_{i} \neq y_{j}, n_{i}, n \in[0,1]$, for all $0 \leq i<j \leq p$, or

- some triple $\langle\geq p R, \triangleleft, n\rangle$ and $x$ has $p R_{\triangleright_{i}, n_{i}}$-neighbours $y_{0}, \ldots, y_{p-1},\left\langle x, y_{i}\right\rangle$ conjugates with $\langle R, \triangleleft, n\rangle$ and $y_{i} \neq y_{j}, n_{i}, n \in[0,1]$, for all $0 \leq i<j \leq p-1$.

Definition $6.7\left(\mathbf{f}_{K D^{-}} \mathcal{S H \mathcal { H } N}\right.$ Tableaux Algorithm) The initialisation of a forest $\left(\mathcal{F}_{\mathcal{A}}\right)$ for an $f_{K D^{-}} \mathcal{S H \mathcal { I N }} A B$ ox $\mathcal{A}$ is similar to the initialisation of a forest for an $f_{K D^{-}} \mathcal{S I} A B o x$ $\mathcal{A}$, with the difference, that equalities and inequalities need to be considered. More precisely, we also initialise the relation $\neq$ as $x_{a_{i}} \neq x_{a_{j}}$ if $a_{i} \neq a_{j} \in \mathcal{A}$ and the relation $\doteq$ to be empty. The latter is used to keep track the nodes that are merged due to the application of a rule for number restrictions. Finally, the algorithm expands $\mathcal{R}$ by adding axioms $\operatorname{Inv}(R) \sqsubseteq \operatorname{lnv}(S)$ for each $R \sqsubseteq S \in \mathcal{R} . \mathcal{F}_{\mathcal{A}}$ is then expanded by repeatedly applying the completion rules from Tables 4 and 6 . Note that in Table 6 we abuse the syntax and use the notation $\operatorname{Inv}(\mathcal{L}(\langle x, y\rangle))$ to indicate the set of triples obtained from $\mathcal{L}(\langle x, y\rangle)$ by applying function Inv to the role $R$ of each triple $\langle R, \bowtie, n\rangle \in \mathcal{L}(\langle x, y\rangle)$.

Example 6.8 Now, let us see some examples of the new expansion rules.

- $\left(\forall_{+}\right):$Let $\langle\forall S . C,>, 0.6\rangle \in \mathcal{L}(x),\langle\operatorname{Inv}(P), \geq, 0.7\rangle \in \mathcal{L}(\langle y, x\rangle)$ with $\operatorname{Trans}(R)$ and $P \sqsubseteq$ $R \sqsubseteq S$. Then, there is role $R$, with $R \sqsubseteq S$, and $y$ is an $R \geq 0.7-n e i g h b o u r$ of $x$, since $y$ is

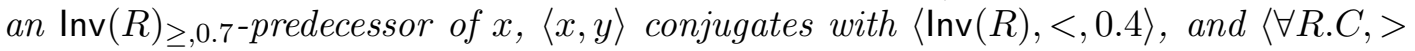
$, 0.6\rangle \notin \mathcal{L}(y)$. Hence, $\langle\forall R . C,>, 0.6\rangle$ should be added in $\mathcal{L}(y)$.

- $(\leq \geq):$ Let $\left.\langle\leq 2 S, \geq, 0.7\rangle \in \mathcal{L}(x),\langle S,>, 0.7\rangle \in \mathcal{L}\left(\left\langle x, y_{1}\right\rangle\right),\langle S\rangle, 0.8,\right\rangle \in \mathcal{L}\left(\left\langle x, y_{2}\right\rangle\right)$ and $\langle P, \geq, 0.4\rangle \in \mathcal{L}\left(\left\langle x, y_{3}\right\rangle\right)$ with $P \stackrel{\varpi}{\underline{E}}$. Hence, $x$ has $3 S_{\triangleright^{\prime} n^{\prime}}$-neighbours all conjugated with $\left\langle S, \geq^{-}, 1-0.7\right\rangle \equiv\langle S, \leq, 0.3\rangle$ and none an ancestor of $x$. Hence we have to nondeterministically merge two of them. If we replace the triple $\langle S,>, 0.7\rangle \in \mathcal{L}\left(\left\langle x, y_{1}\right\rangle\right)$ with $\langle S\rangle, 0.2$,$\rangle the rule is no more applicable. That is because although y_{1}$ is an $S_{\triangleright^{\prime} n^{\prime}}$ neighbour of $x,\left\langle x, y_{1}\right\rangle$ does not conjugate anymore with $\langle S, \leq, 0.3\rangle$. Intuitively, this means that the connection between $x$ and $y_{1}$ is too weak and thus does not contradict the at-most restriction on $x$.

As it is obvious the algorithm can be used in order to perform reasoning for the weaker language $\mathrm{f}_{K D^{-}} \mathcal{S H \mathcal { H } \mathcal { F }}\left(\mathrm{f}_{K D^{-}} \mathcal{S H \mathcal { I }}\right.$ plus functional number restrictions Horrocks \& Sattler, 1999. $\mathcal{S H \mathcal { H } F}$ is obtained from $\mathcal{S H \mathcal { I N }}$ by allowing only cardinalities 0 and 1 in at-most and at-least restriction. It is worth noting that, without counting datatypes, $\mathcal{S H \mathcal { H }} \mathcal{F}$ is the logical underpinning of the OWL Lite ontology language (Horrocks et al., 2003). 


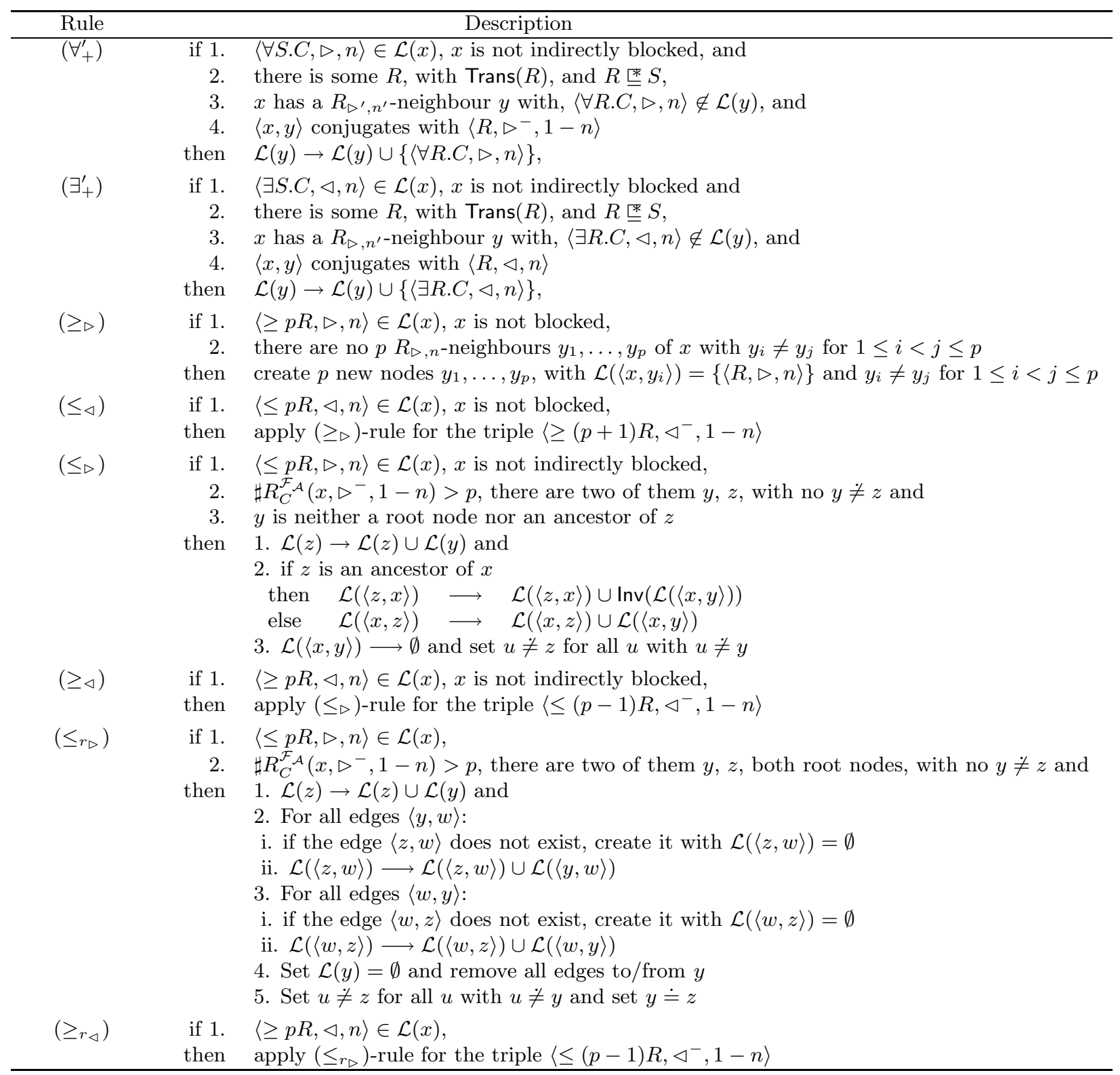

Table 6: Additional tableaux rules for $\mathrm{f}_{K D^{-}} \mathcal{S H \mathcal { H N }}$

\subsection{Decidability of $\mathbf{f}_{K D^{-}} \mathcal{S} \mathcal{H} \mathcal{I} \mathcal{N}$}

The proof of termination, soundness and completeness of $\mathrm{f}_{K D} \mathcal{S} \mathcal{H} \mathcal{I} \mathcal{N}$ is slightly more involved than that of $\mathrm{f}_{K D^{-}} \mathcal{S I}$. This is mainly due to the requirement to apply the unravelling process on a constructed finite completion forest.

Lemma 6.9 (Termination) Let $\mathcal{A}$ be an $f_{K D}-\mathcal{S H I N} A B o x \mathcal{A}$ and $\mathcal{R}$ an $R$ Box. The tableaux algorithm terminates when started for $\mathcal{A}$ and $\mathcal{R}$. 
Proof: Let $m=|s u b(\mathcal{A})|, k=\left|\mathbf{R}_{\mathcal{A}}\right|, p_{\max }=\max \{p \mid \geq p R \in \operatorname{sub}(\mathcal{A})\}$ and $l$ be the number of different membership degrees appearing in $\mathcal{A}$. The termination of our algorithm is a consequence of the same properties that ensure termination in the case of the crisp $\mathcal{S H I \mathcal { N }}$ language (Horrocks et al., 2000). In brief we have the following observations. Firstly, the only rules that remove nodes or concepts from the node labels are the rules $\leq_{\triangleright}, \geq_{\triangleleft}, \leq_{r_{\triangleright}}$ and $\geq_{r_{\triangleleft}}$, which either expand them or set them to $\varnothing$, which means that nodes will be blocked and will remain blocked forever. Secondly, the expansion rules $\left(\exists_{\triangleright}, \geq_{\triangleright}\right.$ and the dual ones for negative inequalities) can only be applied once for each node for the same reasons as in the $\mathcal{S H \mathcal { N } N}$ case (Horrocks et al., 2000). Since $\operatorname{sub}(\mathcal{A})$ contains at most $m$ concepts $\exists R . C$ and $\forall R . C$, the out-degree of the tree is bounded by $2 l m p_{\text {max }}$. Finally, there is a finite number of possible labellings for a pair of nodes and an edge, since concepts are taken from $\operatorname{sub}(\mathcal{A})$ and the number of membership degrees is finite. Thus, there are at most $2^{8 m l k}$ possible labellings for a pair of nodes and an edge. Hence, if a path $p$ is of length at least $2^{8 m l k}$, the pair-wise blocking condition implies that there are 2 nodes $x, y$ on $p$ such that $y$ directly blocks $x$.

Lemma 6.10 (Soundness) If the expansion rules can be applied to an $f_{K D}-\mathcal{S H I N} A B o x$ $\mathcal{A}$ and $R B$ ox $\mathcal{R}$, such that they yield a complete and clash-free completion forest, then $\mathcal{A}$ has a fuzzy tableau w.r.t. $\mathcal{R}$.

Proof: Let $\mathcal{F}_{\mathcal{A}}$ be a complete and clash-free completion forest constructed by the tableaux algorithm for $\mathcal{A}$. Since the $\mathcal{S H \mathcal { I N }}$ language does not have the finite model property (Horrocks \& Sattler, 1999) we have to unravel a possibly blocked tree in order to obtain an infinite tableau. The constructions of such fuzzy tableau works as follows. An individual in $\mathbf{S}$ corresponds to a path in $\mathcal{F}_{\mathcal{A}}$. Moving down to blocked nodes and up to blocking ones we can define infinite such paths. More precisely, a path is a sequence of pairs of nodes of $\mathcal{F}_{\mathcal{A}}$ of the form $p=\left[\frac{x_{0}}{x_{0}^{\prime}}, \ldots, \frac{x_{n}}{x_{n}^{\prime}}\right]$. For such a path we define Tail $(p):=x_{n}$ and Tail $(p):=x_{0}^{\prime}$. With $\left[p \mid \frac{x_{n+1}}{x_{n+1}^{\prime}}\right]$, we denote the path $\left[\frac{x_{0}}{x_{0}^{\prime}}, \ldots, \frac{x_{n}}{x_{n}^{\prime}}, \frac{x_{n+1}}{x_{n+1}^{\prime}}\right]$. The set $\operatorname{Paths}\left(\mathcal{F}_{\mathcal{A}}\right)$ is defined inductively as follows:

- For root nodes $x_{a_{i}}$ of $\mathcal{F}_{\mathcal{A}},\left[\frac{x_{a_{i}}}{x_{a_{i}}}\right] \in \operatorname{Paths}\left(\mathcal{F}_{\mathcal{A}}\right)$, and

- For a path $p \in \operatorname{Paths}\left(\mathcal{F}_{\mathcal{A}}\right)$ and a node $z$ in $\mathcal{F}_{\mathcal{A}}$ :

- if $z$ is a successor of $\operatorname{Tail}(p)$ and $z$ is neither blocked not a root node, then $\left[p \mid \frac{z}{z}\right] \in \operatorname{Paths}\left(\mathcal{F}_{\mathcal{A}}\right)$, or

- if for some node $y$ in $\mathcal{F}_{\mathcal{A}}, y$ is a successor of Tail $(p)$ and $z$ blocks $y$, then $\left[p \mid \frac{z}{y}\right] \in$ $\operatorname{Paths}\left(\mathcal{F}_{\mathcal{A}}\right)$

Please node that since root nodes are never blocked, nor are they blocking other nodes the only place where they occur in a path is in the first place. Moreover, if $p \in \operatorname{Paths}\left(\mathcal{F}_{\mathcal{A}}\right)$, then Tail $(p)$ is not blocked; Tail $(p)=\operatorname{Tail}^{\prime}(p)$ iff Tail' $(p)$ is not blocked and at last $\mathcal{L}(\operatorname{Tail}(p))=$ $\mathcal{L}\left(\right.$ Tail' $\left.^{\prime}(p)\right)$.

Membership degrees are defined exactly as in the case of $\mathrm{f}_{K D^{-}} \mathcal{S I}$. Then, a fuzzy tableau can be defined as in the case of $\mathrm{f}_{K D^{-}} \mathcal{S I}$ with the following differences: 


$$
\begin{aligned}
\mathbf{S}= & \operatorname{Paths}\left(\mathcal{F}_{\mathcal{A}}\right), \\
\mathcal{E}\left(R,\left\langle p,\left[p \mid \frac{x}{x^{\prime}}\right]\right\rangle\right)= & g l b[\langle R, \bowtie, n\rangle],\langle R, \bowtie, n\rangle \in \mathcal{L}\left(\left\langle\text { Tail }(p), x^{\prime}\right\rangle\right) \\
\mathcal{E}\left(R,\left\langle\left[q \mid \frac{x}{x^{\prime}}\right], q\right\rangle\right)= & g l b[\langle\operatorname{lnv}(R), \bowtie, n\rangle],\langle\operatorname{Inv}(R), \bowtie, n\rangle \in \mathcal{L}\left(\left\langle\operatorname{Tail}(q), x^{\prime}\right\rangle\right) \\
\mathcal{E}\left(R,\left\langle\left[\frac{x}{x}\right],\left[\frac{y}{y}\right]\right\rangle\right)= & g l b\left[\left\langle R^{*}, \bowtie, n\right\rangle\right], x, y \text { root nodes and } y \text {-neighbour of } x, \\
\mathcal{V}\left(a_{i}\right)= & \left\{\begin{array}{r}
{\left[\frac{x_{a_{i}}}{x_{a_{i}}}\right] \text { if } x_{a_{i}} \text { is a root node in } \mathcal{F}_{\mathcal{A}} \text { with } \mathcal{L}\left(x_{a_{i}}\right) \neq \emptyset} \\
{\left[\frac{x_{a_{j}}}{x_{a_{j}}}\right] \text { if } \mathcal{L}\left(x_{a_{j}}\right)=\emptyset \text { and } x_{a_{j}} \text { is a root node },} \\
\text { with } \mathcal{L}\left(x_{a_{j}}\right) \neq \emptyset \text { and } x_{a_{i}} \doteq x_{a_{j}}
\end{array}\right.
\end{aligned}
$$

It can be shown that $T$ is a fuzzy tableau for $\mathcal{A}$ w.r.t. $\mathcal{R}$ :

1. Properties 1-6 and Property 13 of Definition 5.1 are satisfied due to the same reasons as in the proof of lemma 5.9 .

2. For property 7 , let $p, q \in \mathbf{S}$ with $\mathcal{L}(p, \forall R . C)=n_{1} \geq n$ and $\mathcal{E}(R,\langle p, q\rangle) \not \leq 1-n$, i.e. $\mathcal{E}(R,\langle p, q\rangle)>1-n$. The definition of $T$ implies that either $\left\langle\forall R . C, \geq, n_{1}\right\rangle \in$ $\mathcal{L}(\operatorname{Tail}(p))$ or $\left\langle\forall R . C,>, n^{\prime}\right\rangle \in \mathcal{L}(\operatorname{Tail}(p))$ with $n_{1}=n^{\prime}+\epsilon$. If $q=\left[p \mid \frac{x}{x^{\prime}}\right]$, then $x^{\prime}$ is an $R$-successor of Tail $(p)$ and, since $g l b$ does not create unnecessary conjugations we have that $\langle R, \triangleright, r\rangle \in \mathcal{L}\left(\left\langle\operatorname{Tail}(p), x^{\prime}\right\rangle\right)$ conjugates with $\langle R, \leq, 1-n\rangle$. Hence, due to completeness of $\mathcal{F}_{\mathcal{A}}$ we have either $\left\langle C, \geq, n_{1}\right\rangle \in \mathcal{L}\left(x^{\prime}\right)$ or $\left\langle C,>, n^{\prime}\right\rangle \in \mathcal{L}\left(x^{\prime}\right)$. From the definition of $\operatorname{Paths}\left(\mathcal{F}_{\mathcal{A}}\right)$ we have that $\mathcal{L}\left(x^{\prime}\right)=\mathcal{L}(x)=\mathcal{L}(q)$. If $p=\left[q \mid \frac{x}{x^{\prime}}\right]$, then $x^{\prime}$ is an $\operatorname{Inv}(R)$-successor of Tail $(q)$ and again, the definition of $g l b$ implies that $\langle\operatorname{Inv}(R), \triangleright, r\rangle \in$ $\mathcal{L}\left(\left\langle\operatorname{Tail}(q), x^{\prime}\right\rangle\right)$ conjugates with $\langle\operatorname{Inv}(R), \leq, 1-n\rangle$. Thus, due to completeness of $\mathcal{F}_{\mathcal{A}}$, either $\left\langle C, \geq, n_{1}\right\rangle \in \mathcal{L}(\operatorname{Tail}(q))=\mathcal{L}(q)$ or $\left\langle C,>, n^{\prime}\right\rangle \in \mathcal{L}(\operatorname{Tail}(q))=\mathcal{L}(q)$. If $p=\left[\frac{x}{x}\right]$ and $q=\left[\frac{y}{y}\right]$ for two root nodes $x, y$ then $y$ is an $R$-neighbour of $x$, and since the $\forall_{\triangleright}$-rule does not apply we have that wither $\left\langle C, \geq, n_{1}\right\rangle \in \mathcal{L}(y)=\mathcal{L}(q)$ or $\left\langle C,>, n^{\prime}\right\rangle \in \mathcal{L}(y)=\mathcal{L}(q)$. Similar proof holds for $\mathcal{L}(p, \forall R . C)>n$ and for property 8 , of definition 5.1 and for the modified properties $11^{\prime}$ and 12 ' of definition 6.4 .

3. For property 9 of Definition 5.1, assume that $\mathcal{L}(p, \exists R . C)=n_{1} \geq n$ and let Tail $(p)=x$. The definition of $T$ implies that either $\left\langle\exists R . C, \geq, n_{1}\right\rangle \in \mathcal{L}(x)$ or $\left\langle\exists R . C,>, n^{\prime}\right\rangle \in \mathcal{L}(x)$, with $n_{1}=n^{\prime}+\epsilon$. We have to show that there is some $q \in \mathbf{S}$ such that $\mathcal{E}(R,\langle p, q\rangle) \geq$ $n_{1} \geq n$ and $\mathcal{L}(q, C) \geq n_{1} \geq n$. Since the $\exists_{\triangleright}$-rule is not applicable there is some $y$ in $\mathcal{F}_{\mathcal{A}}$ with either $\left\langle C, \geq, n_{1}\right\rangle \in \mathcal{L}(y)$ or $\left\langle C,>, n^{\prime}\right\rangle \in \mathcal{L}(y)$. Now there are two possibilities:

(a) If $y$ is a successor of $x$, then $y$ can either be a root node or not. In case $y$ is a root node so is $x$, since it is a predecessor of $y$, so $p=\left[\frac{x}{x}\right]$ and $q=\left[\frac{y}{y}\right] \in \mathbf{S}$. In case $y$ is not a root node if $y$ is not blocked, then $q=\left[p \mid \frac{y}{y}\right] \in \mathbf{S}$; if $y$ is blocked by some $z$ then, $q=\left[p \mid \frac{z}{y}\right] \in \mathbf{S}$.

(b) $x$ is an $\operatorname{lnv}(R)$-successor of $y$. Since $x$ is a successor of $y$ we distinguish the cases of $x$ being a root node or not. If $x$ is a root then so is $y$, hence $q=\left[\frac{y}{y}\right] \in \mathbf{S}$. If $x$ is not a root node then either $p=\left[q \mid \frac{x}{x^{\prime}}\right]$, with Tail $(q)=y$, or $p=\left[q \mid \frac{x}{x^{\prime}}\right]$, with Tail $(q)=u \neq y, x$ blocks $x^{\prime}$ and $u$ is a predecessor of $x^{\prime}$. By the definition of pair-wise blocking we have that $\mathcal{L}(y)=\mathcal{L}(u)$ and $\mathcal{L}(\langle y, x\rangle)=\mathcal{L}\left(\left\langle u, x^{\prime}\right\rangle\right)$.

In any of these cases, $\mathcal{E}(R,\langle p, q\rangle) \geq n_{1} \geq n, \mathcal{L}(q, C) \geq n_{1} \geq n$. Similar proof applies for $\mathcal{L}(p, \exists R . C)>n$ and for property 10 . 
4. Property 16 in definition 6.4 is satisfied due to the definition of $R$-successor that takes into account the role hierarchy.

5. For Property 17 assume that $\mathcal{L}(p, \geq m R)=n_{1} \geq n$. The definition of $T$ implies that either $\left\langle\geq m R, \geq, n_{1}\right\rangle \in \mathcal{L}(x)$ or $\left\langle\geq m R,>, n^{\prime}\right\rangle \in \mathcal{L}(x)$, with $n_{1}=n^{\prime}+\epsilon$. This means that there are $m$ individuals $y_{1}, \ldots, y_{m}$ in $\mathcal{F}_{\mathcal{A}}$ such that each $y_{i}$ is an $R_{\geq, n^{\prime}}$ or $R_{>, n^{\prime}}$-neighbour of $x$. We have to show that for each of these $y_{i} \mathrm{~s}$, there is a path $q_{i}$, such that $\mathcal{E}\left(R,\left\langle p, q_{i}\right\rangle\right) \geq n_{1}$, and $q_{i} \neq q_{j}$ for all $1 \leq i<j \leq m$. The proof is similar with the one given by Horrocks et al. (2000). It is based on the fact that in case where some $z$ blocks several $y_{i}$ s, then the construction of the paths distinguishes between these $y_{i}$ s be seting $q_{i}=\left[p \mid \frac{z}{y_{i}}\right]$, thus ensuring the existence of different paths in $T$. Thus, for each $y_{i}$ there is a different path $q_{i}$ in $\mathbf{S}$ with $\mathcal{E}\left(R,\left\langle p, q_{i}\right\rangle\right) \geq n_{1} \geq n$, or $\mathcal{E}\left(R,\left\langle p, q_{i}\right\rangle\right) \geq n^{\prime}+\epsilon \geq n$ and $\sharp R^{T}(p, \geq, n) \geq m$. Similarly for $\mathcal{L}(p, \geq m R)>n$ and for property 18 .

6. For Property 19 in definition 6.4 suppose that there exists $p \in \mathbf{S}$ with $\mathcal{L}(p, \leq m R)=$ $n_{1} \geq n$ and $\sharp R_{\neg}^{T}(p, \leq, 1-n)>m$. We have to show that this implies $\sharp R_{C}^{\mathcal{F}_{\mathcal{A}}}(\operatorname{Tail}(p), \leq$ $, 1-n)>m$, in the completion-forest, thus contradicting either clash-freeness or completeness of $\mathcal{F}_{\mathcal{A}}$. More precisely, one has to show that the construction does not create more conjugated paths for $T$ than those that exist in $\mathcal{F}_{\mathcal{A}}$. This can only be the case if for some node $y$ the construction creates two distinct paths of the form $q_{i}=\left[p \mid \frac{y_{i}}{y}\right]$. As shown by Horrocks et al. (2000), the proof relies on the fact that the function Tail' is injective on the paths of $T$, i.e. for $q_{1}$ and $q_{2}$, Tail' $\left(q_{1}\right)=y=\operatorname{Tail}^{\prime}\left(q_{2}\right)$ implies that $q_{1}=q_{2}$. Hence, such paths cannot be distinct. Similar observations hold for $\mathcal{L}(p, \leq m S)>n$ and for property 20 .

7. Properties 14 and 15 of Definition 5.1 are satisfied cause of the initialization of the completion-forest and the fact that the algorithm never blocks root nodes. Furthermore, for each root node $x_{a_{i}}$ whose label and edges are removed by the $\leq_{r_{\triangleright}}$-rule, there is another root node $x_{a_{j}}$ with $x_{a_{i}}=x_{a_{j}}$ and $\left\{\langle C, \triangleright, n\rangle \mid\left(a_{i}: C\right) \triangleright n \in \mathcal{A}\right\} \subseteq \mathcal{L}\left(x_{a_{j}}\right)$.

8. Property 21 of Definition 6.4 is satisfied because the $\leq_{r_{\triangleright}}$-rule does not identify two root nodes $x_{a_{i}}, x_{a_{j}}$ when $x_{a_{i}} \neq x_{a_{j}}$ holds.

Lemma 6.11 (Completeness) Let $\mathcal{A}$ be an $f_{K D^{-}} \mathcal{S H \mathcal { H } N}$ fuzzy $A$ Box and $\mathcal{R}$ an $R$ Box. If $\mathcal{A}$ has a fuzzy tableau w.r.t. $\mathcal{R}$, then the expansion rules can be applied to $\mathcal{A}$ and $\mathcal{R}$ in such a way that the tableaux algorithm yields a complete and clash-free completion-forest.

Proof: The proof is quite similar with the proof of lemma 5.10. In the new algorithm we have some new non-deterministic rules, but again the existence of fuzzy tableau for $\mathcal{A}$ w.r.t. $\mathcal{R}$ can help us steer the application of those non-deterministic rules. In the following table we show the modified rule $\leq_{\triangleright}^{\prime}$. The rest of the non-deterministic rules can be guided by modifying them in a similar way. 


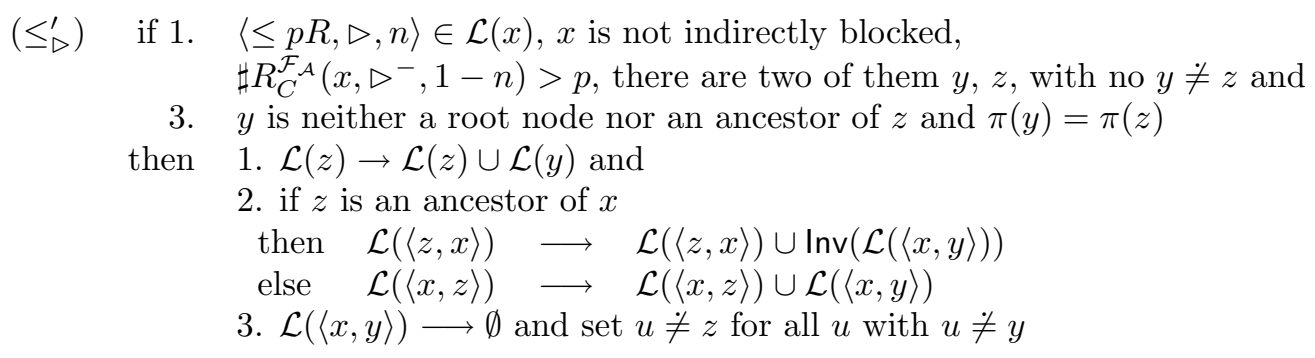

Table 7: The $\leq_{\triangleright}^{\prime}$-rule

Theorem 6.12 The tableaux algorithm is a decision procedure for the consistency problem

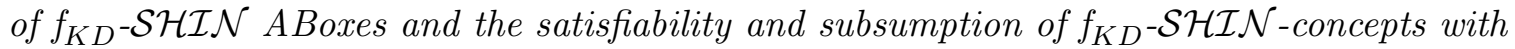
respect to simple terminologies.

We will conclude this section by investigating the complexity of the proposed algorithm.

Lemma 6.13 For an $f_{K D^{-}} \mathcal{S H \mathcal { I N }} A B$ ox $\mathcal{A}$ and a role hierarchy $\mathcal{R}$, $\operatorname{sub}(\mathcal{A}, \mathcal{R})=\mathcal{O}(|\mathcal{A}| \times$ $|\mathcal{R}|)$.

Proof: The proof is quite similar with the one presented by Tobies $(2001)$. Since $\operatorname{sub}(\mathcal{A}, \mathcal{R})$ contains all concepts $C$ such that $(a: C) \bowtie n \in \mathcal{A}$ and is closed under sub-concepts of $C$, it contains $\mathcal{O}(|\mathcal{A}|)$ concepts. Additionally, we have to add a concept $\forall R . C$ or $\exists R . C$ to $\operatorname{sub}(\mathcal{A}, \mathcal{R})$ if $\forall S . R \in \operatorname{sub}(\mathcal{A}, \mathcal{R})$ or $\exists S . R \in \operatorname{sub}(\mathcal{A}, \mathcal{R})$ and $R \stackrel{乛}{=}$ and then $\operatorname{close} \operatorname{sub}(\mathcal{A}, \mathcal{R})$ again under sub-concepts and $\sim$. This may yield at most two concept for every concept in $\operatorname{sub}(\mathcal{A}, \mathcal{R})$ and role in $\mathcal{R}$. Thus, $\operatorname{sub}(\mathcal{A}, \mathcal{R})=\mathcal{O}(2|\mathcal{A}| \times|\mathcal{R}|)$.

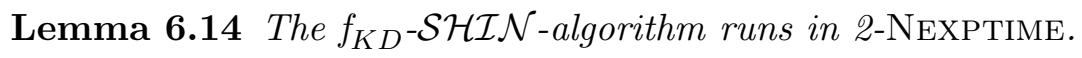

Proof: Let $\mathcal{A}$ be a $\mathrm{f}_{K D^{-}} \mathcal{S} \mathcal{H} \mathcal{I N}$ ABox and $\mathcal{R}$ an RBox. Let $m=\operatorname{sub}(\mathcal{A}), k=\left|\mathbf{R}_{\mathcal{A}}\right|$, $p_{\max }$ the maximum number $p$ that occurs in a number restriction and $l$ the number of different membership degrees appearing in $\mathcal{A}$. Following Tobies (2001) we set $n=|\mathcal{A}|+|\mathcal{R}|$, then due to lemma 6.13 it holds that $m=\mathcal{O}(2|\mathcal{A}| \cdot|\mathcal{R}|)=\mathcal{O}\left(n^{2}\right), k=\mathcal{O}(|\mathcal{A}|+|\mathcal{R}|)$, $p_{\text {max }}=\mathcal{O}\left(2^{|\mathcal{A}|}\right)=\mathcal{O}\left(2^{n}\right)$ and $l=\mathcal{O}(|\mathcal{A}|)=\mathcal{O}(n)$. In the proof of lemma 6.9 we have shown that paths in a completion-forest for $\mathcal{A}$ become no longer than $2^{8 m l k}$ and that the out-degree is bounded by $2 l m p_{\max }$. Hence, the $\mathrm{f}_{K D^{-}} \mathcal{S} \mathcal{H} \mathcal{I N}$ algorithm will construct a forest with no more than

$$
\left(2 l m p_{\max }\right)^{2^{8 m l k}}=\mathcal{O}\left(\left(2 n \cdot n^{2} \cdot 2^{n}\right)^{2^{8 n^{2} \cdot n \cdot n}}\right)=\mathcal{O}\left(2^{n \cdot 2^{8 n^{4}}}\right)=\mathcal{O}\left(2^{2^{n^{5}}}\right)
$$

nodes.

Hence, the $\mathrm{f}_{K D^{-}} \mathcal{S} \mathcal{H} \mathcal{I N}$ algorithm is of the same theoretical complexity as the $\mathcal{S H \mathcal { I N }}$ algorithm (Tobies, 2001).

Concluding our presentation on the issue of reasoning with expressive fuzzy DLs we comment on how to handle GCIs in the $\mathrm{f}_{K D^{-}} \mathcal{S} \mathcal{I}$ and $\mathrm{f}_{K D^{-}} \mathcal{S H \mathcal { I } \mathcal { N }}$ languages. As it is noted by Horrocks and Sattler (1999), $\mathcal{S H \mathcal { I N }}$ is expressive enough to internalize GCIs into a single concept, hence reducing reasoning with GCIs to concept satisfiability. The idea 
behind internalization is that the semantic restrictions imposed by an axioms of the form $C \sqsubseteq D$ can be encoded within a concept of the form $\neg C \sqcup D$. As it was remarked by Stoilos et al. (2006) this reduction of concept inclusions does not hold for $\mathrm{f}_{K D}$-DLs, since the semantics of the axiom $C \sqsubseteq D$ are different than that of the concept $\neg C \sqcup D$. Hence, the internalization method proposed by Horrocks and Sattler (1999) for the $\mathcal{S H \mathcal { I N }}$ language cannot be applied in the $\mathrm{f}_{K D^{-}} \mathcal{S} \mathcal{H} \mathcal{I} \mathcal{N}$ language.

Stoilos et al. (2006) and Li et al. (2006a) propose techniques by which we can handle GCIs in $\mathrm{f}_{K D^{-}}$-DLs. Stoilos et al. (2006) use the DL language $\mathrm{f}_{K D^{-}} \mathcal{A} \mathcal{L C}$ in order to present their technique, while $\mathrm{Li}$ et al. (2006a) use the language $\mathrm{f}_{K D}-\mathcal{S H} \mathcal{I}$. These procedures can be applied in the cases of $\mathrm{f}_{K D}-\mathcal{S} \mathcal{I}$ and $\mathrm{f}_{K D}-\mathcal{S H \mathcal { I N }}$, since they are independent of the underlying DL formalism. Roughly speaking these techniques are performed in three steps. In the first step the ABox is normalized, by replacing each assertion of the form $(\mathrm{a}: C)>n$ and $(\mathrm{a}: C)<n$ by assertions $(\mathrm{a}: C) \geq n+\ell$ and $(\mathrm{a}: C) \leq n-\ell$, respectively, where $\ell$ is a small number from $[0,1]$. Obviously, in a normalized ABox only assertions with inequalities $\geq$ and $\leq$ are present. In the second step the set of relative membership degrees is constructed: $X^{\mathcal{A}}=\{0,0.5,1\} \cup\{n, 1-n \mid \phi \bowtie n\}$, where obviously $\bowtie \in\{\geq, \leq\}$. Finally, a tableaux expansion rule is employed to transfer the semantic restrictions imposed by each GCI $C \sqsubseteq D \in \mathcal{T}$ into fuzzy assertions of the ABox. More precisely, for each $C \sqsubseteq D \in \mathcal{T}$, node $x$ in $\mathcal{F}_{\mathcal{A}}$ and degree $n \in X^{\mathcal{A}}$, the algorithm adds either $\langle C, \leq, n-\ell\rangle$ or $\langle D, \geq, n\rangle$ to $\mathcal{L}(x)$. We remark here that the rule proposed by Li et al. (2006a) is slightly different.

As noted by Stoilos et al. (2006), tableaux algorithms need to be slightly changed in order to handle GCIs. First, due to the normalization step, degrees are now taken from the interval $[-\ell, 1+\ell]$, thus clash definitions $\langle\perp\rangle, n$,$\rangle and \langle\top,<, n\rangle$ are removed since no assertion with $>$ and $<$ exist anymore and the clashes $\langle C,<, 0\rangle$ and $\langle C,>, 1\rangle$ are replaced by $\langle C, \leq,-\ell\rangle$ and $\langle C, \geq, 1+\ell\rangle$, respectively. The termination of the algorithm is not affected since again the set of membership degrees is finite (taken from the set $X^{\mathcal{A}}$ ), but the practical complexity increases dramatically since we have a non-deterministic choice for each axiom $C \sqsubseteq D \in \mathcal{T}$ and degree $n \in X^{\mathcal{A}}$. The proof of soundness is not affected much and as it was showed by Stoilos et al. (2006) the $g l b$ function is replaced by a simple max, due to the lack of assertions with inequalities $>$ and $<$, while the non-deterministic rule for handling subsumptions can also be modified to be guided, in order to provide us with a proof for completeness.

Example 6.15 Let the knowledge base $\Sigma=\langle\{\geq 1 R \sqsubseteq C\},\{(\langle\mathrm{a}, \mathrm{b}\rangle: R) \geq 0.6,(\mathrm{a}: C)<$ $0.6\}\rangle$. Intuitively, the concept axioms states that the domain of the role $R$ is concept $C .^{5}$ Obviously, the knowledge base is unsatisfiable since the concept axiom suggests that $\forall x^{\mathcal{I}} \in$ $\Delta^{\mathcal{I}}, \sup _{b_{1}^{\mathcal{I}}} \min _{i=1}^{1}\left(R^{\mathcal{I}}\left(x^{\mathcal{I}}, b_{i}^{\mathcal{I}}\right)\right)=R^{\mathcal{I}}\left(x^{\mathcal{I}}, c^{\mathcal{I}}\right) \leq C^{\mathcal{I}}\left(x^{\mathcal{I}}\right)$, for some arbitrary $c^{\mathcal{I}} \in \Delta^{\mathcal{I}}$, but the $A B o x$ assertions state that there exists $\mathrm{a}^{\mathcal{I}} \in \Delta^{\mathcal{I}}$ and $b^{\mathcal{I}} \in \Delta^{\mathcal{I}}$ such that $R^{\mathcal{I}}\left(\mathrm{a}^{\mathcal{I}}, b^{\mathcal{I}}\right) \geq 0.6>$ $C^{\mathcal{I}}\left(\mathrm{a}^{\mathcal{I}}\right)$. The above concept inclusion axioms is a GCI, hence we have to use a technique for GCIs.

First, we apply the normalization step in the original ABox, obtaining the normalized one: $\{(\langle\mathrm{a}, \mathrm{b}\rangle: R) \geq 0.6,(\mathrm{a}: C) \leq 0.6-\ell\}$. Secondly, we collect the set of relative membership degrees: $X^{\mathcal{A}}=\{0,0.5,1\} \cup\{0.4,0.4+\ell, 0.6-\ell, 0.6\}$.

5. A domain axiom can also be stated as $\exists R . \top \sqsubseteq C$, but we use the above form in order to show how the algorithm behaves with number restrictions. 
Then, the algorithm initializes a completion-forest to contain the following nodes with the respective triples:

$$
\begin{aligned}
& \text { (1) }\langle R, \geq, 0.6\rangle \in \mathcal{L}\left(\left\langle x_{\mathrm{a}}, x_{\mathrm{b}}\right\rangle\right) \\
& \text { (2) }\langle C, \leq, 0.6-\ell\rangle \in \mathcal{L}\left(x_{\mathrm{a}}\right)
\end{aligned}
$$

Then the algorithm expands the completion forest by using the rules from Tables 4 and 6 and with the additional rule presented by Stoilos et al. (2006). This rule applied on the axiom $\geq 1 R \sqsubseteq C$ adds either $\langle\geq 1 R, \leq, n-\ell\rangle$ or $\langle C, \geq, n\rangle$ in $\mathcal{L}\left(x_{\mathrm{a}}\right)$, for each $n \in X^{\mathcal{A}}$. Hence, at some point the algorithm chooses $0.6 \in X^{\mathcal{A}}$ and adds either $\langle\geq 1 R, \leq, 0.6-\ell\rangle$, or $\langle C, \geq, 0.6\rangle$. In the former case $\langle\geq 1 R, \leq, 0.6-\ell\rangle \in \mathcal{L}\left(x_{\mathrm{a}}\right)$ and $x_{\mathrm{a}}$ has $1 R_{\geq, 0.6}$-neighbour $x_{\mathrm{b}}$, and $\left\langle x_{\mathrm{a}}, x_{\mathrm{b}}\right\rangle$ conjugated with $\langle R, \leq, 0.6-\ell\rangle$, while in the latter case $\{\langle C, \leq, 0.6-\ell\rangle,\langle C, \geq, 0.6\rangle\} \subseteq \mathcal{L}\left(x_{\mathbf{a}}\right)$, hence $\mathcal{L}\left(x_{\mathrm{a}}\right)$ contains a pair of conjugated triples and thus a clash. We conclude that all possible expansions result to a clash, thus the knowledge base is unsatisfiable.

\section{Related Work}

There have been many efforts in the past to extend description logics with fuzzy set theory (Yen, 1991; Tresp \& Molitor, 1998; Straccia, 2001; Hölldobler et al., 2002; Sanchez \& Tettamanzi, 2006; Straccia, 2005b; Hajek, 2005; Li, Xu, Lu, \& Kang, 2006b). The first effort was presented by Yen (1991). In his extension, explicit membership functions over a domain were used as well as membership manipulators, like "very" or "moreOrLess", in order to alter membership functions and define new concepts from already defined ones. A later approach was presented by Tresp and Molitor (1998), where membership manipulators also appear. Regarding reasoning algorithms Yen described a structural subsumption algorithm for a rather small DL language while Tresp and Molitor a tableaux calculus for $\mathcal{A L C}_{F M}$ $(\mathcal{A L C}$ extended with fuzzy set theory and the membership manipulator constructor). The application of the tableaux rules creates a set of equations and inequations which are later solved with an optimization method. Moreover, when determining a subsumption or entailment relation between two concepts, with respect to a $(\mathrm{KB})$, the assertions of the KB were considered of a crisp form (i.e. $a$ belongs to $C$ to a degree of 1 ). After the application of the reasoning algorithm and the solution of the equations the minimum value of the solution set is taken as the degree that the KB entails the crisp assertion or that a concept is subsumed by another.

A fuzzy extension of the $\mathcal{A L C}$ language was also considered by Straccia $(2001,1998)$. Reasoning algorithms for the problem of crisp entailment and subsumption were provided, and were based on tableaux calculus. The algorithm was proved to be PSPACE-complete. Moreover, complete reasoning algorithms for fuzzy $\mathcal{A L C}$ were provided by Hölldobler et al. (2002), where membership manipulators (linguistic hedges) were also used on primitive concepts. This approach was later extended by Hölldobler, Nga, and Khang (2005) to allow linguistic hedges also on complex concepts. The languages presented are called $\mathcal{A} \mathcal{L C} \mathcal{C}_{F H}$ and $\mathcal{A L C}_{F L H}$ ( $\mathcal{A L C}$ plus linguistic hedges and linear linguistic hedges, respectively). In all these approaches also the min-max norms and the Kleene-Dienes implication were used to perform the fuzzy set theoretic operations. 
Approaches towards more expressive DLs, are presented by Sánchez and Tettamanzi (2004), Sanchez and Tettamanzi (2006), Straccia (2005b), Straccia (2005a) and Stoilos et al. (2005c). The language considered by Sánchez and Tettamanzi (2004) is $\mathcal{A L C Q}(\mathcal{A L C}$ plus qualified number restrictions, Tobies, 2001). The authors also include fuzzy quantifiers which is a novel approach to fuzzy DLs, and the norm operations are the same with the ones used here. Subsequently, Sanchez and Tettamanzi (2006) propose a procedure to calculate the satisfiability interval for a fuzzy concept. Due to the presence of fuzzy quantifiers it is not clear how other inference problems, like entailment and subsumption can be solved. Straccia

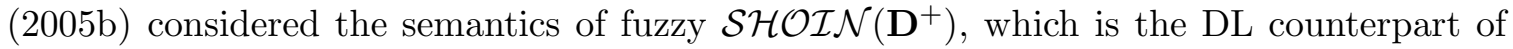
the OWL DL language. In his approach generalized norm operations were used for the semantics, while no reasoning algorithms were provided as well as no investigation of the properties of value and existential restrictions, when transitive relations and role hierarchies participate in such concepts. Furthermore, the semantics of number restrictions were not analyzed. The approach by Straccia was used by Stoilos et al. (2005c), in order to provide the abstract syntax and semantics to concept and role descriptions and axioms of the fuzzy OWL language. Additionally, Stoilos et al. (2005c) present a method to translate a fuzzy OWL ontology to a fuzzy $\mathcal{S H O \mathcal { N }}$ knowledge base, thus reasoning in fuzzy OWL can be reduced to reasoning in expressive fuzzy DLs. At last the language considered by Straccia (2005a) is $\mathcal{A L C}(\mathcal{D})(\mathcal{A L C}$ plus concrete domains), where additionally a reasoning algorithm based on an optimization technique was presented. The norm operations used are the ones we used in the current paper, plus the Lukasiewicz t-norm, $t(a, b)=\max (0, a+b-1)$, t-conorm, $u(a, b)=\min (1, a+b)$ and fuzzy implication $\mathcal{J}(a, b)=\min (1,1-a+b)$. An approach towards fuzzy DLs with concrete domains has been also presented by Liu, Tian, and Ma (1994) for modelling the selection of research and development projects.

In all previous approaches reasoning with respect to simple and acyclic TBoxes was considered. Stoilos et al. (2006) propose a method to perform reasoning w.r.t. general and/or cyclic TBoxes in the language $\mathrm{f}_{K D}-\mathcal{A L C}$. This method applies a preprocessing step on the ABox, called normalization and then it extends the classical $\mathrm{f}_{K D}-\mathcal{A L C}$ algorithm (Straccia, 2001) with an additional rule, in order to deal with general and cyclic axioms. Moreover, Li et al. (2006a) extend the fuzzy tableau of $\mathrm{f}_{K D^{-}} \mathcal{S H \mathcal { I }}$ proposed by Stoilos et al. (2005a) with an additional rule to also handle with general and cyclic TBoxes in the language $\mathrm{f}_{K D^{-}} \mathcal{S H \mathcal { I }}$. Interestingly, the technique used by Stoilos et al. (2006) is different than that presented by Li et al. (2006a).

It also is worth noting the works of Bonatti and Tettamanzi (2005), where the complexity of fuzzy DL languages is investigated. Furthermore, Hajek (2005) investigates properties of the fuzzy $\mathcal{A L C}$ language, when arbitrary continuous norm operations are used and provides interesting results. More precisely, Hajek shows that the problems of concept satisfiability and subsumption are decidable for the Lukasiewicz fuzzy $\mathcal{A L C}\left(\mathrm{f}_{L}-\mathcal{A L C}\right)$, while in product fuzzy $\mathcal{A L C}\left(\mathrm{f}_{P}-\mathcal{A L C}\right)$ and Gödel fuzzy $\mathcal{A L C}\left(\mathrm{f}_{G}-\mathcal{A L C}\right)$ only witnessed satisfiability and subsumption are decidable. For unrestricted models both $\mathrm{f}_{P}-\mathcal{A L C}$ and $\mathrm{f}_{G}-\mathcal{A L C}$ lack the finite model property (Hajek, 2005). This is accomplished by reducing these problems to the problem of propositional satisfiability of fuzzy propositional logic. These results where further extended to fuzzy DLs with truth constants, i.e. to ABox consistency, again by Hajek (2006). Moreover, Straccia (2004) present a technique by which an $\mathrm{f}_{K D}$ - $\mathcal{A L C H}$ knowledge base can be reduced to a crisp $\mathcal{A L C H}$ knowledge base. Hence, reasoning in a fuzzy $\mathrm{KB}$ 
can be performed by using existing and optimized DL systems. Then, Bobillo et al. (2006)

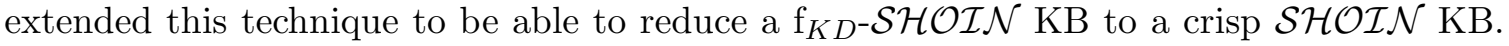
At last, Li, Xu, Lu, Kang, and Wang (2005) and Li et al. (2006b), also use the idea of the reduction in order to annotate the concepts and roles of the crisp languages $\mathcal{A L C N}$ and $\mathcal{A L C} \mathcal{Q}$, respectively with degrees denoted as sub-scripts in the syntax of concepts and roles and provide reasoning for the languages $\mathrm{f}_{K D^{-}} \mathcal{A L C \mathcal { N }}$ and $\mathrm{f}_{K D^{-}} \mathcal{A L C} \mathcal{Q}$.

In all previous approaches, reasoning algorithms for rather inexpressive fuzzy DLs, i.e. fuzzy- $\mathcal{A L C}$ extended with concept modifiers or concrete domains or number restrictions or qualified number restrictions or general TBoxes were presented. As far as we know this is the first presentation of a reasoning algorithm for such complex fuzzy DL languages. In order to achieve our goal we have provided an investigation on the semantics of the extended language when fuzzy transitive relations and role hierarchies are considered in value and existential restrictions or of the number restrictions constructor. The aim of such an investigation is to discover if properties of the classical $\mathcal{S I}$ and $\mathcal{S H \mathcal { N }}$ languages, like the propagation of value restrictions or counting of $R$-neighbours also apply in the fuzzy case. We have found that apart from value restrictions also existential restrictions have to be propagated. Additionally, we have shown that the membership degree of these concepts in their new nodes is the same as that in their source nodes. Moreover, we have seen that role hierarchies can be smoothly integrated as in the classical case. Additionally, the analysis of the semantics of number restrictions have shown that despite how complex their semantics might appear, regarding reasoning they can also be efficiently handled, as in the classical case. Furthermore, we have investigated the applicability of blocking strategies, like dynamic blocking and pair-wise blocking, which are used in the crisp $\mathcal{S I}$ and $\mathcal{S H I N}$ language to ensure the termination of the proposed algorithms. We have seen that the properties of the norm operations used ensure that such blocking conditions can also be applied. Based on these investigations, we were able to provide a tableaux reasoning algorithm, to decide the key inference problems of very expressive fuzzy DLs, and we have proved their soundness, completeness and termination.

\section{Conclusions and Future Work}

Making applications capable of coping with vagueness and imprecision will result in the creation of systems and applications which will provide us with high quality results and answers to complex user defined tasks. To this direction we have to extend with fuzzy set theory the underlying logical formalisms that they use in order to represent knowledge and perform reasoning tasks. DL is a logical formalism that has gained a lot of attention the last decade, cause of its decidability, the powerful reasoning tools that have been implemented and the well-defined model-theoretic semantics.

Towards extending DLs with fuzzy set theory we have presented two very expressive fuzzy DLs, $\mathrm{f}_{K D^{-}} \mathcal{S} \mathcal{I}$ and $\mathrm{f}_{K D^{-}} \mathcal{S} \mathcal{H} \mathcal{I} \mathcal{N}$. We have investigated the properties of the semantics that result by adding fuzziness to very expressive fuzzy DLs, i.e. to fuzzy DLs that allow for transitive and inverse roles, role hierarchies and number restrictions and we have provided sound, complete and terminating reasoning algorithms for both of these formalisms. Even though handling fuzziness in such expressive languages seems quite difficult and reasoning was not previously known, we show that $\mathrm{f}_{K D^{-}} \mathcal{S} \mathcal{I}$ and $\mathrm{f}_{K D^{-}} \mathcal{S H \mathcal { I } \mathcal { N }}$ with the min, max norms 
are still decidable. We have shown that the techniques used in the classical case can also be applied in the extended frameworks, but this can only happen after closely investigating the properties of the languages and after proving that these techniques also work in this new setting. In the current paper we have not addressed nominals $(\mathcal{O})$ (Horrocks \& Sattler, 2005). Note that in the fuzzy DL literature, there are proposals for crisp nominals (Stoilos et al., 2005c) and fuzzy nominals (Bobillo et al., 2006). Thus, the nominal constructor is not yet a mature notion in fuzzy DLs and more research is needed in order to find appropriate semantics for them, considering also the issue from the application point of view.

As far as future directions are concerned, these will include the extension of the algorithm of $\mathrm{f}_{K D^{-}} \mathcal{S H \mathcal { I } N}$, in order to provide reasoning support for the fuzzy DL $\mathrm{f}_{K D^{-}} \mathcal{S H O I} \mathcal{Q}$. $\mathcal{S H O I Q}$ extends $\mathcal{S H I N}$ with qualified number restrictions (Tobies, 2001), which are very important in real life applications (Rector \& Horrocks, 1997), and with nominals. Thus, we also intend to compare the properties of the different proposals for nominals in fuzzy DLs. Again, although we expect that similar notions as in the classical $\mathcal{S H O} \mathcal{H} \mathcal{Q}$ language

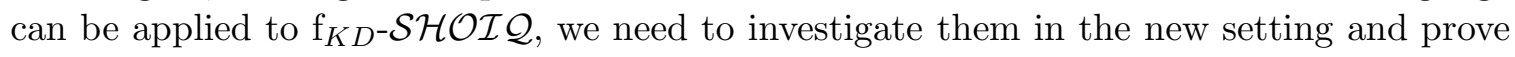
that they work. Furthermore, additional research effort can be focused on the investigation of the reasoning problem for the $f-\mathcal{S I}$ and $f-\mathcal{S H I N}$ languages, extended with other norm

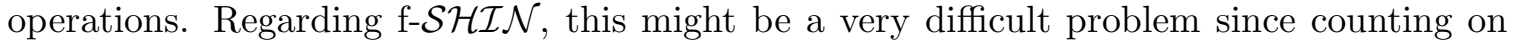
number restrictions might not be possible anymore.

\section{Acknowledgments}

This work is supported by the FP6 Network of Excellence EU project Knowledge Web (IST2004-507482). Giorgos Stoilos, Giorgos Stamou and Vassilis Tzouvaras were also partially funded by the European Commission under FP6 Integrated Project X-Media (FP6-026978).

\section{References}

Alejandro, J., Belle, T., \& Smith, J. (2003). Modal keywords, ontologies and reasoning for video understanding. In Proceedings of the International Conference on Image and Video Retrieval.

Athanasiadis, T., Mylonas, P., Avrithis, Y., \& Kollias, S. (2007). Semantic image segmentation and object labeling. IEEE Transactions on Circuits and Systems for Video Technology, 17(3), 298-312.

Baader, F. (1990). Augmenting Concept Languages by Transitive Closure of Roles: An Alternative to Terminological Cycles. Research report RR-90-13. An abridged version appeaered in Proc. IJCAI-91,pp.446-451.

Baader, F., McGuinness, D., Nardi, D., \& Patel-Schneider, P. (2002a). The Description Logic Handbook: Theory, implementation and applications. Cambridge University Press.

Baader, F., Horrocks, I., \& Sattler, U. (2002b). Description Logics for the Semantic Web. KI - Künstliche Intelligenz, 16(4), 57-59. 
Bechhofer, S., van Harmelen, F., Hendler, J., Horrocks, I., McGuinness, D. L., PatelSchneider, P. F., \& eds., L. A. S. (2004). OWL web ontology language reference. Tech. rep..

Benitez, A. B., Smith, J. R., \& Chang, S. (2000). MediaNet: a multimedia information network for knowledge representation. In Proc. SPIE Vol. 4210, p. 1-12, Internet Multimedia Management Systems, John R. Smith; Chinh Le; Sethuraman Panchanathan; C.-C. J. Kuo; Eds., pp. 1-12.

Berners-Lee, T., Hendler, J., \& Lassila, O. (2001). The semantic web. Scientific American.

Bobillo, F., Delgado, M., \& Gómez-Romero, J. (2006). A crisp representation for fuzzy shoin with fuzzy nominals and general concept inclusions. In Proc. of the 2nd International Workshop on Uncertainty Reasoning for the Semantic Web (URSW 06), Athens, Georgia.

Bonatti, P., \& Tettamanzi, A. (2005). Some complexity results on fuzzy description logics. In V. Di Gesù, F. Masulli, A. P. (Ed.), WILF 2003 International Workshop on Fuzzy Logic and Applications, LNCS 2955, Berlin. Springer Verlag.

Calvanese, D., De Giacomo, G., Lenzerini, M., Nardi, D., \& Rosati, R. (1998). Description logic framework for information integration. In Proc. of the 6th Int. Conf. on the Principles of Knowledge Representation and Reasoning (KR'98), pp. 2-13.

Chen, H., Fellah, S., \& Bishr, Y. (2005). Rules for geospatial semantic web applications. In W3C Workshop on Rule Languages for Interoperability.

Ding, Z., \& Peng, Y. (2004). A Probabilistic Extension to Ontology Language OWL. In Proceedings of the 37th Hawaii International Conference On System Sciences (HICSS37)., p. 10, Big Island, Hawaii.

Dubois, D., \& Prade, H. (2001). Possibility theory, probability theory and many-valued logics: A clarification. Ann. Math. Artif. Intell., 32(1-4), 35-66.

Fagin, R. (1998). Fuzzy queries in multimedia database systems. In Proc. Seventeenth ACM Symp. on Principles of Database Systems, pp. 1-10.

Giugno, R., \& Lukasiewicz, T. (2002). P-SHOQ(D): A probabilistic extension of shoq(d) for probabilistic ontologies in the semantic web. In JELIA '02: Proceedings of the European Conference on Logics in Artificial Intelligence, pp. 86-97, London, UK. Springer-Verlag.

Hajek, P. (1998). Metamathematics of fuzzy logic. Kluwer.

Hajek, P. (2005). Making fuzzy description logic more general. Fuzzy Sets and Systems, $154(1), 1-15$.

Hajek, P. (2006). Computational complexity of $t$-norm based propositional fuzzy logics with rational truth constants. Fuzzy Sets and Systems, 157(13), 677-682.

Hölldobler, S., Khang, T. D., \& Störr, H.-P. (2002). A fuzzy description logic with hedges as concept modifiers. In Proceedings InTech/VJFuzzy'2002, pp. 25-34.

Hölldobler, S., Nga, N. H., \& Khang, T. D. (2005). The fuzzy description logic $\mathcal{A L C}_{F L H}$. In International workshop on Description Logics. CEUR. 
Hollunder, B. (1994). An alternative proof method for possibilistic logic and its application to terminological logics. In Proceedings of the 10th Annual Conference on Uncertainty in Artificial Intelligence (UAI-94), pp. 327-335, San Francisco, CA. Morgan Kaufmann Publishers.

Hollunder, B., Nutt, W., \& Schmidt-Schaus, M. (1990). Subsumption algorithms for concept description languages. In European Conference on Artificial Intelligence, pp. 348-353.

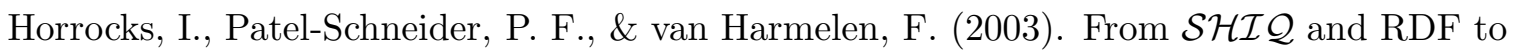
OWL: The making of a web ontology language. Web Semantics, 1.

Horrocks, I., \& Sattler, U. (1999). A description logic with transitive and inverse roles and role hierarchies. Journal of Logic and Computation, 9, 385-410.

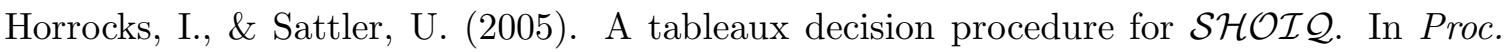
19th Int. Joint Conf. on Artificial Intelligence (IJCAI 05).

Horrocks, I., Sattler, U., \& Tobies, S. (1999). Practical reasoning for expressive description logics. In Proceedings of the 6th International Conference on Logic for Programming and Automated Reasoning (LPAR'99), No. 1705 in LNAI, pp. 161-180. SpringerVerlag.

Horrocks, I., Sattler, U., \& Tobies, S. (2000). Reasoning with Individuals for the Description Logic $\mathcal{S H \mathcal { I }}$. In MacAllester, D. (Ed.), CADE-2000, No. 1831 in LNAI, pp. 482-496. Springer-Verlag.

Kandel, A. (1982). A Fuzzy Techniques in Pattern Recognition. Wiley.

Klement, E. P., Mesiar, R., \& Pap, E. (2004). Triangular norms. position paper I: basic analytical and algebraic properties. Fuzzy Sets and Systems, 143, 5-26.

Klir, G. J., \& Yuan, B. (1995). Fuzzy Sets and Fuzzy Logic: Theory and Applications. Prentice-Hall.

Koller, D., Levy, A., \& Pfeffer, A. (1997). P-CLASSIC: A tractable probabilistic Description Logic. In Proceedings of the 14th National Conference on Artificial Intelligence (AAAI-97)., pp. 390-397.

Krishnapuram, R., \& Keller, J. (1992). Fuzzy set theoretic approach to computer vision: An overview. In IEEE International Conference on Fuzzy Systems, pp. 135-142.

Larsen, H., \& Yager, R. (1993). The use of fuzzy relational thesauri for classificatory problem solving in information restrieval and exprert systems. IEEE Trans. in System, Man, and Cybernetics, 23(1), 31-41.

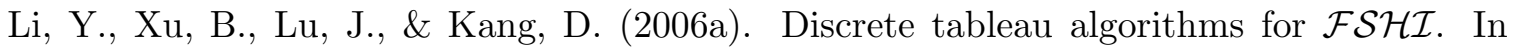
Proceedings of the International Workshop on Description Logics (DL 2006), Lake District, UK.

Li, Y., Xu, B., Lu, J., \& Kang, D. (2006b). Reasoning technique for extended fuzzy $\mathcal{A} \mathcal{L C} \mathcal{Q}$ . In $\operatorname{ICCSA}$ (2), pp. 1179-1188.

Li, Y., Xu, B., Lu, J., Kang, D., \& Wang, P. (2005). Extended fuzzy description logic $\mathcal{A} \mathcal{L C N}$. In Proceedings of the 9th International Conference on Knowledge Based Intelligent Information and EngineeringSystems (KES-05), pp. 896-902. 
Liu, O., Tian, Q., \& Ma, J. (1994). A fuzzy description logic approach to model management in r\&d project selection. In Proceedings of the 8th Pacific Asian Conference on Information Systems (PACIS-04).

McGuiness, D. (2003). Configuration. In Baader, F., Calvanese, D., McGuinness, D., Nardi, D., \& Patel-Schneider, P. F. (Eds.), The Description Logic Handbook: Theory, Implementation, and Applications, pp. 388-405. Cambridge University Press.

Meghini, C., Sebastiani, F., \& Straccia, U. (2001). A model of multimedia information retrieval. Journal of the ACM, 48(5), 909-970.

Mostert, P., \& Shields, A. (1957). On the structure of semigroups on a compact manifold with boundary. The Annals of Mathematics, 65(1), 117-143.

Navara, M. (2000). Satisfiability in fuzzy logic. Neural Network World, 10(5), 845-858.

Nebel, B. (1990). Terminological reasoning is inherently intractable. Journal of Artificial Intelligence, 43, 235-249.

Oguntade, O., \& Beaumont, P. (1982). Ophthalmological prognosis via fuzzy subsets. Fuzzy Sets and Systems, 7(2), 123-179.

Pan, J. Z. (2004). Description Logics: Reasoning Support for the Semantic Web. Ph.D. thesis, School of Computer Science, The University of Manchester, Oxford Rd, Manchester M13 9PL, UK.

Rector, A. L., \& Horrocks, I. (1997). Experience building a large, re-usable medical ontology using a description logic with transitivity and concept inclusions. In Proceedings Workshop on Ontological Engineering, AAAI Spring Symposium, Stanford CA., pp. 100-107. Hanley and Belfus, Inc., Philadelphia, PA.

Sánchez, D., \& Tettamanzi, A. (2004). Generalizing quantification in fuzzy description logic. In Proceedings 8th Fuzzy Days in Dortmund.

Sanchez, D., \& Tettamanzi, A. A. (2006). Fuzzy quantification in fuzzy description logics. In Sanchez, E. (Ed.), Capturing Intelligence: Fuzzy Logic and the Semantic Web. Elsevier.

Sattler, U. (1996). A concept language extended with different kinds of transitive roles. In KI '96: Proceedings of the 20th Annual German Conference on Artificial Intelligence, pp. 333-345. Springer-Verlag.

Stoilos, G., Stamou, G., Tzouvaras, V., Pan, J., \& Horrocks, I. (2005a). The fuzzy description logic f-SHIN . In Proceedings of the International Workshop on Uncertainty Reasoning for the Semantic Web.

Stoilos, G., Stamou, G., Tzouvaras, V., Pan, J., \& Horrocks, I. (2005b). A fuzzy description logic for multimedia knowledge representation. In Proc. of the International Workshop on Multimedia and the Semantic Web.

Stoilos, G., Stamou, G., Tzouvaras, V., Pan, J., \& Horrocks, I. (2005c). Fuzzy OWL: Uncertainty and the semantic web. In Proc. of the International Workshop on OWL: Experiences and Directions. 
Stoilos, G., Straccia, U., Stamou, G., \& Pan, J. (2006). General concept inclusions in fuzzy description logics. In Proceedings of the 17th International Conference on Artificial Intelligence (ECAI 06), pp. 457-461. IOS Press.

Straccia, U. (2001). Reasoning within fuzzy description logics. Journal of Artificial Intelligence Research, 14, 137-166.

Straccia, U. (2005a). Description logics with fuzzy concrete domains. In 21st Conf. on Uncertainty in Artificial Intelligence (UAI-05), Edinburgh.

Straccia, U. (2005b). Towards a fuzzy description logic for the semantic web. In Proceedings of the 2nd European Semantic Web Conference.

Straccia, U. (1998). A fuzzy description logic. In AAAI '98/IAAI '98: Proceedings of the fifteenth national/tenth conference on Artificial intelligence/Innovative applications of artificial intelligence, pp. 594-599. American Association for Artificial Intelligence.

Straccia, U. (2004). Transforming fuzzy description logics into classical description logics. In Proceedings of the 9th European Conference on Logics in Artificial Intelligence (JELIA-04), No. 3229 in Lecture Notes in Computer Science, pp. 385-399, Lisbon, Portugal. Springer Verlag.

Sugeno, M. (1985). Industrial Applications of Fuzzy Control. North-Holland.

Tarski, A. (1956). Logic, Semantics, Metamathemetics: Papers from 1923 to 1938. Oxford University Press.

Tobies, S. (2001). Complexity Results and Practical Algorithms for Logics in Knowledge Representation. Ph.D. thesis, Rheinisch-Westfälischen Technischen Hochschule Aachen. URL http://lat.inf.tu-dresden.de/research/phd/Tobies-PhD-2001.pdf .

Tresp, C., \& Molitor, R. (1998). A description logic for vague knowledge. In In proc of the 13th European Conf. on Artificial Intelligence (ECAI-98).

Vardi, M. Y. (1997). Why is modal logic so robustly decidable?. In DIMACS Series in Discrete Mathematics and Theoretical Computer Science, pp. 149-184.

Yen, J. (1991). Generalising term subsumption languages to fuzzy logic. In In Proc of the 12th Int. Joint Conf on Artificial Intelligence (IJCAI-91), pp. 472-477.

Zadeh, L. A. (1965). Fuzzy sets. Information and Control, 8, 338-353.

Zimmermann, H. (1987). Fuzzy Sets, Decision Making, and Expert Systems. Kluwer, Boston. 